\title{
EFFECTS OF PLANT-BASED DIETS ON THE DISTAL GUT MICROBIOME OF RAINBOW TROUT (Oncorhynchus mykiss).
}

\author{
Submitted to the College of Graduate Studies and \\ Research of the University of Saskatchewan in partial \\ fulfillment of the requirements for the degree of \\ Doctor of Philosophy in the Department of Veterinary \\ Microbiology at the University of Saskatchewan.
}

By

ATUL RAVINDRANATH DESAI

(C) Copyright Atul R. Desai, March, 2012

All rights reserved 


\section{PERMISSION TO USE}

In agreement with the outlines set out by the College of Graduate Studies and Research at the University of Saskatchewan, I allow the University of Saskatchewan Libraries to make this thesis available to all interested parties. Also in accordance with the College of Graduate Studies and Research, I allow this thesis to be copied "in any manner, in whole or in part, for scholarly purposes". This thesis may not, however, be reproduced or used in any manner for financial gain without my written consent. Any scholarly use of this thesis, in part or in whole, must acknowledge both myself and the University of Saskatchewan.

Any requests for copying or using this thesis, in any form or capacity, should be made to:

Head of Department of Veterinary Microbiology

University of Saskatchewan

Saskatoon, Saskatche wan

S7N 5B4 


\section{ABSTRACT}

Replacement of fish meal in aquaculture diets with plant proteins is economically desirable, but the effects of these alternative protein sources on fish intestinal microbiota are poorly understood. The fish intestine is colonized by a diverse population of micro-organisms of which bacteria form the biggest component. The intestinal microbiota plays important roles in digestion, pathogen exclusion, maintenance of intestinal homeostasis, and in the development of the immune system of the fish. The purpose of this thesis was to characterise the intestinal microbiota of rainbow trout (Oncorhynchus mykiss) fed with diets based on plant ingredients. Semi quantitative (clone library analysis and 454 pyrosequencing) and quantitative (qRT-PCR) techniques based on the cpn60 gene, and denaturing gradient gel electrophoresis (DGGE) based on the 16S rRNA were used to characterise the intestinal microbiota and evaluate the effects of plant based experimental diets on the structure of the intestinal microbiota. A study was also conducted to evaluate the extent to which individual fish in a recirculating aquaculture system share strains of bacteria, to understand the effect that a shared recirculating facility might have on our ability to resolve differences in the intestinal microbiota of fish fed with different diets. Regardless of the technique used, the intestinal microbiota of fish fed with plant based diets were always distinguishable from fish fed with fish meal diets. Intestinal microbiota of fish fed with plant based diets had higher Firmicutes:Proteobacteria ratios compared to their corresponding controls. This trend was seen with both cpn60 clone library analysis of pooled samples of 
intestinal contents, and cpn60 based pyrosequencing of intestinal microbiota from individual fish. Evaluation of the effects of plant based diets on the intestinal microbiota by DGGE and pyrosequencing showed that the microbiomes of fish fed with plant protein concentrate diets were less distinguishable from fish meal control diet associated microbiomes, than were microbiomes of fish fed with plant meal based diets. $\mathrm{GTG}_{5}$ rep PCR analysis of Carnobacterium maltaromaticum isolates showed that even though the fish were reared in a recirculating facility, individual fish hosted distinct $C$. maltaromaticum strains, suggesting a minimal level of sharing of bacterial strains between fish housed in a common environment. Quantitative real-time PCR was used to determine the abundance of specific bacterial targets in fish intestinal content s associated with particular plant based diets in two replicate trials, to determine if the diets had reproducible effects on the composition of intestinal microbiota. Even though the diets did not have a consistent, reproducible effect at the species level, it was observed that intestinal microbiota composition was always modified with change in diet. Results of these studies indicate that inclusion of plant ingredients to replace fish meal alters the structure of the intestinal microbiota of rainbow trout. Additionally, the protein concentrate forms of the experimental ingredients reduce the impact of plant ingredients on the intestinal microbiota structure making them favourable candidates for fish meal replacement. 


\section{ACKNOWLEDGMENTS}

I wish to express gratitude to my supervisor, Dr Janet E. Hill for her unwavering encouragement and never-ending support during my entire $\mathrm{PhD}$ program. It is on the account of the scientific training that she has imparted on me, that I wish and I hope to continue and contribute to science. Her patience and active quidance alone has brought me this far. Being her first graduate student was an absolute joy where she set an unmatched example of how to balance social life with research. My many thanks to her for making me realize what education truly means, and this to me, goes far more than what ever I have learnt about science.

I thank the members of my PhD advisory committee (Drs. Manuel ChirinoTrejo, Andrew Van kessel, David Janz, Tim Dumonceaux and Vikram Misra) for their quidance, constructive criticism and encouragement. I would like to thank Dr Bonnie Chaban, Matthew Links and Champika Fernando for their support and technical assistance during my research work. Special thanks to all the members of Hill lab and extended hill lab family for their help and company.

Lastly and most importantly I want to thank my Baba and Aai, my elder brother and sister in law for their patience, love, support and encouragment. Aai and Baba I am here because of your sacrifices and I love you very much.

I want to dedicate this thesis to my wife Prachi. You are my inspiration and meaning of life. 


\section{TABLE OF CONTENTS}

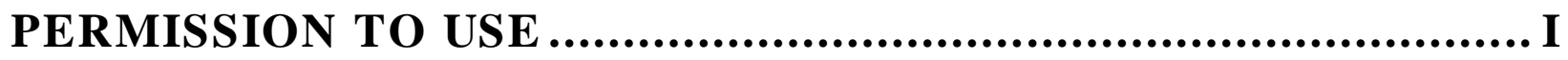

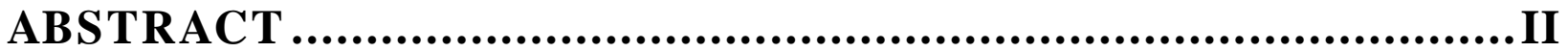

ACKNOWLEDGMENTS .....................................................IV

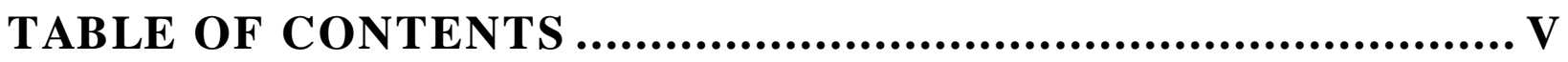

LIST OF TABLES …...........................................................

LIST OF FIG URES …............................................................ XII

Chapter 1 LITERATURE REVIEW ...........................................1

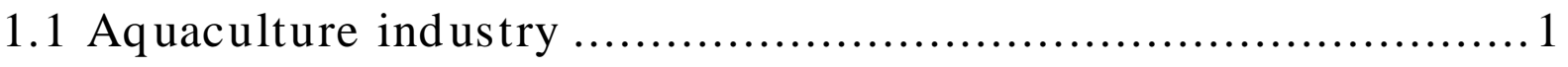

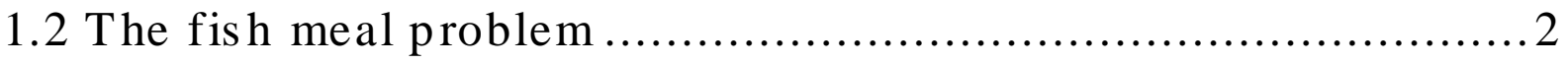

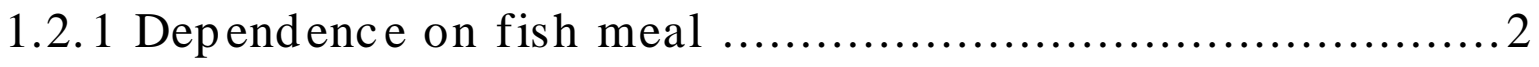

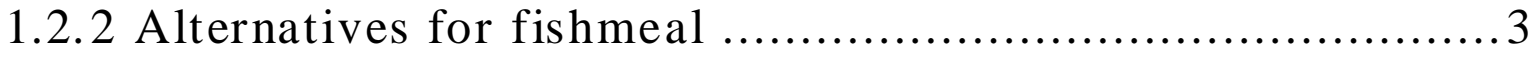

1.3 Challenges and limitations of including plant ingredients ........5

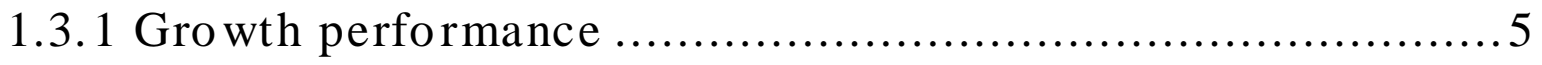

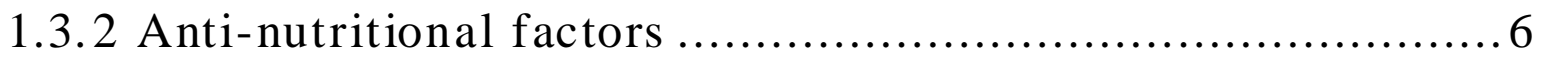

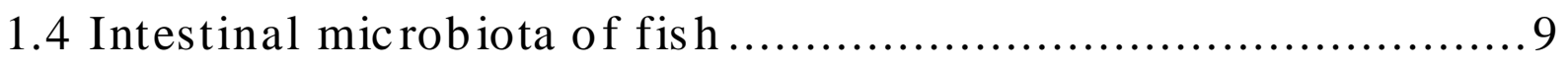

1.4.1 Beneficial role of intestinal microbiota ........................ 10 
1.4.2 Culture based characteris ation of fish intestinal microbiota 11

1.4.3 Culture independent characterisation of fish intestinal

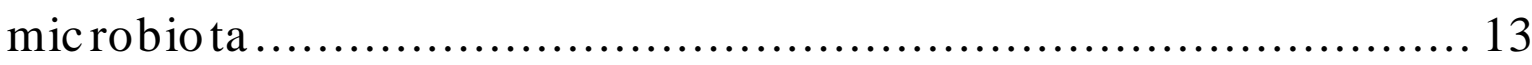

1.4.5 Intestinal microbiota of salmonid fis h......................... 19

Chapter 2 Hypothesis and objectives: .......................................23

Chapter 3 Characterization of rainbow trout (Oncorhynchus mykiss) intestinal microbiota and inflammatory marker gene expression in a recirculating aquaculture system .............................................24

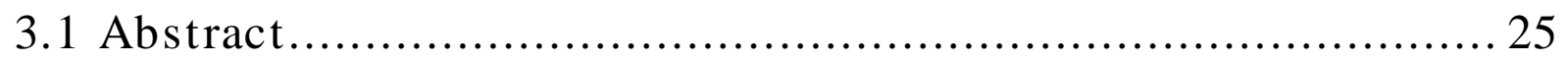

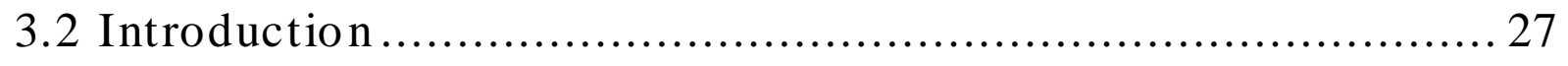

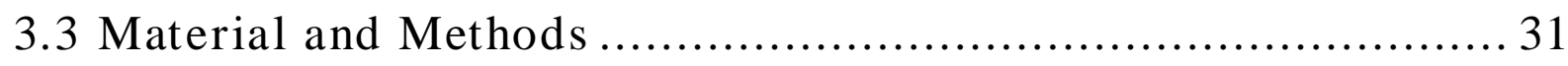

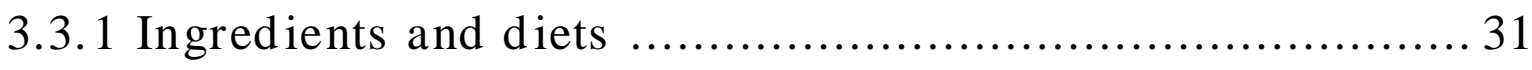

3.3.2 Fish husbandry and digestibility trial design ................. 33

3.3.3 Digestibility analys is ........................................... 34

3.3.4 Intestinal contents and tissue collection ........................ 35

3.3.5 Nucleic acid extraction ......................................... 35

3.3.6 cpn60 clone libraries ................................................ 36 
3.3.7 Quantitative real-time PCR

3.3.8 Statistical analys is 40

3.4 Results 41

3.4.1 Digestibility. 41

3.4.2 cpn60 clone libraries 43

3.4.3 Quantitative PCR of intestinal microbiota 48

3.4.4 Host gene expression 50

3.4.5 Correlations between diets, host gene expression and intestinal microbiota 52

3.5 Discussion 55

Chapter 4 Effects of plant-based diets on the distal gut microbiome of rainbow trout (Oncorhynchus mykiss).

\section{4}

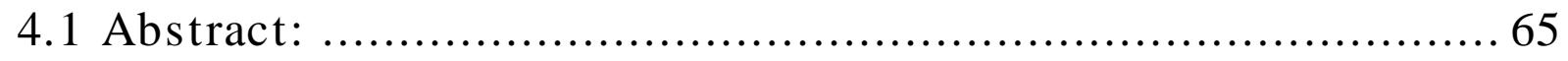

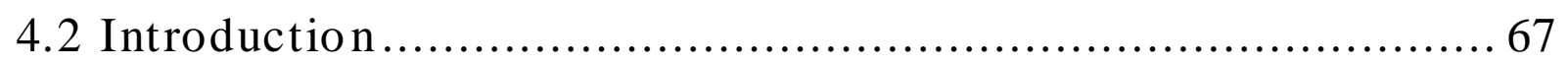

4.3 Materials and methods ............................................. 70

4.3.1 Fish Management ............................................ 70

4.3.2 Growth Experiments ..................................... 70 
4.3.3 Sample collection and DNA extraction 74

4.3.4 16S rRNA PCR-DGGE and cluster analys is .74

4.3.5 Sample preparation for pyrosequencing 75

4.3.6 Sequence data analys is 75

4.4 Results 78

4.4.1 Sequence data and ecological metrics 78

4.4.2 Changes in FM control diet associated microbiota 79

4.4.3 Effects of plant ingredient inclusion on intestinal microbiota 87

4.5 Discussion 94

Chapter 5 Application of quantitative real-time PCR for validation of metagenomic sequencing libraries and investigation of the reproducibility of plant ingredient effects on the rainbow trout intestinal microbiome. 101

5.1 Abstract: 102

5.2 Introduction: 103

5.3 Material and methods: 107 
5.3.1 Fish husbandry

5.3.2 Diets 107

5.3.3 Sample collection and DNA extraction 111

5.3.4 Quantitative real-time PCR 111

5.3.5 16S rRNA PCR-DGGE and cluster analys is 116

5.4 Results: 117

5.4.1 Validating pyrosequencing findings using qRT-PCR :..... 117

5.4.2 qRT-PCR based comparison of target abundance in Trial-1 and Trial-2.

5.4.3 Evaluating effects of plant ingredient based diets on intestinal microbiota using $16 \mathrm{~S}$ rRNA based DGGE: 124

5.5 Discussion: 127

Chapter 6 Discrimination of Carnobacterium maltaromaticum strains isolated from rainbow trout (Onchorhynchus mykiss) intestinal contents fed with different plant based diets.

6.1 Abstract: 134

6.2 Introduction: 135 
6.3 Materials and methods:

6.3.1 Fish husbandry, diets and sample collection: .............. 138

6.3.2 C. maltaromatic um isolation: ................................ 139

6.3 .3 cpn60 PCR and sequencing: .............................. 141

6.3.4 DNA extraction and $\mathrm{GTG}_{5}$ rep PCR: ..................... 141

6.3.5 Cluster analys is :......................................... 142

6.4 R esults : ........................................................... 143

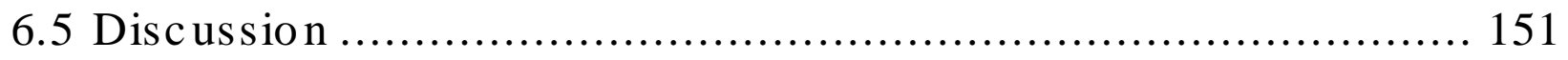

Chapter 7 Summary and conclusions .................................. 155

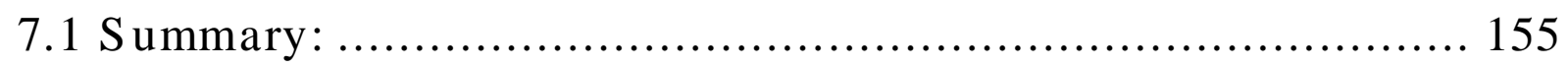

7.2 Conclus ions : ..................................................... 160

Chapter 8: References ..................................................161 


\section{LIST OF TABLES}

Table 3.1 Ingredient composition and nutrient analysis of diets ( $\mathrm{g} \mathrm{kg}-1)$.

Table 3.2 qPCR assays for intestinal gene expression analysis and quantification

of bacteria.

Table 3.3 Apparent digestibility coefficients for experimental diets and ingredients in rainbow trout (dry matter basis).

Table 3.4 Unique cpn60 sequences detected in $\mathrm{cn}$, fm and $\mathrm{sb}$ libraries.

Table 3.5 Expression of target genes in intestine of rainbow trout fed control (cn), fish meal $(\mathrm{fm})$, and soybean meal $(\mathrm{sbm})$ diets $(\mathrm{n}=9$ for each diet $)$.

Table 3.6 Two-tailed correlations between diets, intestinal gene expression and intestinal bacterial populations. Only significant correlations $(p<0.05)$ are shown.

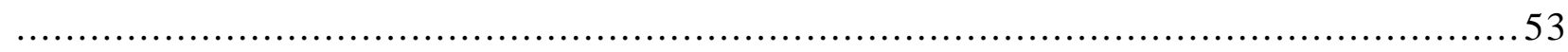

Table 4.1 Ingredient composition of experimental diets.

Table 5.1 Ingredient composition of experimental diets.

Table 5.2 Ingredient composition of experimental diets for trial-2............. 110

Table 5.3 qRT-PCR assays for quantification of bacteria.

Table 6.1 Number of Carnobacterium maltaromaticum strain isolated from distal intestinal contents of rainbow trout fed with different diets. 140 


\section{List of figures}

Figure 3.1 Phylogenetic tree based on the 32 unique sequences detected in the CN, FM and SB libraries and related reference strain sequences

Figure 3.2 Quantitative PCR detection of four bacterial targets in intestinal contents of individual fish comprising the cpn60 library pools.

Figure 3.3 The relationships between significant correlations $(P<0.05)$ of diets, intestinal gene expression and intestinal bacterial populations in rainbow trout. 54

Figure 4.1 Timing of diet trials. Horizontal bars indicate the 8 -week period for each stage of the experiment. 73

Figure 4.2 Rarefaction curves of cpn60 UT amplicon libraries of intestinal microbiota of fish fed with different plant-based diets (upper panel) and FM control diets (lower panel). 80

Figure 4.3 Box plots (median, quartiles and range values) of (A) Chao 1 richness and (B) Simpson's diversity index of intestinal microbiota of fish fed with different diets.

Figure 4.4 DGGE fingerprints of distal gut contents of individual fish fed with the FM control diet during the course of experiment..... 82

Figure 4.5 DGGE fingerprints of distal gut contents of individual fish fed with experimental diets. .83 
Figure 4.6 $16 \mathrm{~S}$ rRN A gene PCR-DGGE analysis of the distal gut content samples obtained from fish fed with the FM control diet over the course of the experiment.

Figure 4.7 Relationships of FM control diet associated profiles determined by cpn60 UT pyrosequencing. 86

Figure 4.8 Proportional abundance of bacterial phyla represented in cpn60 UT amplicon libraries derived from the intestinal contents of rainbow trout. 89

Figure 4.9 Box plots (median, quartiles and range values) of Firmicutes:Proteobacteria ratios for individual fish fed experimental and control diets 90

Figure 4.10 Clustering of DGGE fingerprint profiles of intestinal microbiota of rainbow trout associated with experimental diet and concurrent controls. 91

Figure 4.11 Principal coordinates analysis based on weighted UniFrac distance. White squares indicate fish fed with FM control diet and black circles indicate fish fed the experimental diet.

Figure 5.1 Comparis on of qRT-PCR copy numbers (bars, left ordinate) and target sequence frequency (squares, right ordinate) of (A) FIR-1, (B) FIR-2 and (C) FIR-3 from Trial- 1.

Figure 5.2 Comparison of qRT-PCR copy numbers (bars, left ordinate) and target sequence frequency (squares, right ordinate) of (A) PRO-1, (B) PRO-2 (C) FIR 3 and (D) PRO-4 from Trial-1. 121 
Figure 5.3 Comparison of the copy numbers of the seven bacterial targets in pooled intestinal contents of fish fed with experimental diets (A) PM, (B) CM and (C) SBM in Trial-1 and Trial-2, determined by qRT-PCR

Figure 5.4 Cluster analysis of 16S rRNA DGGE profiles from individual fish fed with PM (top), CM (middle) or SBM (bottom) during Trial-2 ...................... 125

Figure 5.5 Clustering of 16S rRNA DGGE profiles of individual rainbow trout fed PM (top), CM (middle) or SBM (bottom) in Trial-1 and Trial-2. Trees are collapsed at the lowest common node for each diet group.

Figure 6.1 Dendograms derived from $\mathrm{GTG}_{5}$ rep PCR fingerprints of C. maltaromaticum isolates from distal gut contents of individual fish reared during the PM diet trial. 144

Figure 6.2 Dendograms derived from $\mathrm{GTG}_{5}$ rep PCR fingerprints of $C$. maltaromaticum isolates from distal gut content of individual fish reared during the SPC diet trial. Labels indicate fish number. 145

Figure 6.3 Dendograms derived from $\mathrm{GTG}_{5}$ rep PCR fingerprints of $C$. maltaromaticum isolates from distal gut content of individual fish reared during the $\mathrm{CM}$ diet trial. Labels indicate fish number.

Figure 6.4 Dendograms derived from $\mathrm{GTG}_{5}$ rep PCR fingerprints of $C$. maltaromaticum isolates from distal gut content of individual fish reared during the CPC diet trial. 147 
Figure 6.5 Dendograms derived from $\mathrm{GTG}_{5}$ rep PCR fingerprints of $C$. maltaromaticum isolates from distal gut contents of all fish reared during (A) PM, (B) SPC, (C) CM, and (D) CPC diet trials..................................... 150 


\begin{tabular}{|c|c|}
\hline ABBREVIATIONS & THIS THESIS \\
\hline${ }^{\circ} \mathrm{C}$ & Degrees Celcius \\
\hline$\mu \mathrm{l}$ & Microlitre \\
\hline ADC & Apparent digestibility coefficients \\
\hline AIA & Acid insoluble ash \\
\hline ATCC & American Type Culture Collection \\
\hline$\beta$-act & Beta actin \\
\hline C. maltaromaticum & Carnobacterium maltaromaticum \\
\hline $\mathrm{CCAC}$ & Canadian Council on Animal Care \\
\hline cDNA & Complementary deoxyribonucleic acid \\
\hline cfu & Colony forming unit \\
\hline $\mathrm{CM}$ & Canola meal \\
\hline $\mathrm{CM}_{\mathrm{T} 2}$ & Canola meal diet fed during Trial-2 \\
\hline $\mathrm{CN}$ & Control \\
\hline $\mathrm{CPC}$ & Canola protein concentrate \\
\hline cpn60 & Chaperonin-60 \\
\hline cpnDB & Chaperonin-60 databse \\
\hline CTSI Agar & Cresol red thallium acetate sucrose inulin agar \\
\hline DGGE & Denaturing gradient gel electrophoresis \\
\hline DMSO & Dime thylsulfoxide \\
\hline DNA & Deoxyribonucleic acid \\
\hline DNQ & Detectable but not quantifiable \\
\hline dNTP & deoxy N (A-C-G-T) triphosphate \\
\hline E. feacalis & Enterococcus feacalis \\
\hline FAO & Food and Agriculture Organization \\
\hline FIR & Firmic ute \\
\hline FISH & Fluorescence in situ hybridisation \\
\hline FM & Fish meal \\
\hline $\mathrm{FM}_{\mathrm{CM}}$ & Fishmeal corresponding to $\mathrm{CM}$ \\
\hline $\mathrm{FM}_{\mathrm{CPC}}$ & Fishmeal corresponding to CPC \\
\hline
\end{tabular}




\begin{tabular}{|c|c|}
\hline $\mathrm{FM}_{\mathrm{PM}}$ & Fishmeal corresponding to PM \\
\hline $\mathrm{FM}_{\mathrm{PPC}}$ & Fishmeal corresponding to PPC \\
\hline $\mathrm{FM}_{\mathrm{SBM}}$ & Fishmeal corresponding to SBM \\
\hline $\mathrm{FM}_{\mathrm{SPC}}$ & Fishmeal corresponding to SPC \\
\hline $\mathrm{FM}_{\mathrm{T} 2}$ & Fishmeal diet fed during Trial-2 \\
\hline $\mathrm{g}$ & Gram \\
\hline GI & Gastrointestinal \\
\hline $\mathrm{GTG}_{5}$ rep $\mathrm{PCR}$ & $\mathrm{GTG}_{5}$ Repetitive polymerase chain reaction \\
\hline H. alvae & Hafnia alvae \\
\hline $\operatorname{IgM}$ & Immunog lobulin $\mathrm{M}$ \\
\hline IL- $1 \beta$ & Interleukin- 1 beta \\
\hline $\mathrm{kDa}$ & Kilodalton \\
\hline L. citreum & Leuconostoc citreum \\
\hline MID & Multiplexing identification \\
\hline $\mathrm{nM}$ & Nanomolar \\
\hline NRC & National Research Council \\
\hline NSP & Non-starch polysaccharide \\
\hline O. mykiss & Onchorhynchus mykiss \\
\hline OTU & Operational taxonomic unit \\
\hline PCNA & Proliferating cell nuclear antigen \\
\hline $\mathrm{PCoA}$ & Principal coordinates analysis \\
\hline PCR & Polymerase chain reaction \\
\hline $\mathrm{PM}$ & Pea meal \\
\hline $\mathrm{PM}_{\mathrm{T} 2}$ & Pea meal diet fed during Trial-2 \\
\hline PPC & Pea protein concentrate \\
\hline PRO & Proteobacteria \\
\hline qRT-PCR & Quantitative real-time PCR \\
\hline RFLP & Restriction fragment length polymorphism \\
\hline rRNA & Ribosomal ribonucleic acid \\
\hline SBM & Soybean meal \\
\hline
\end{tabular}


$\mathrm{SBM}_{\mathrm{T} 2}$

SEM

SPC

TGGE

t-RFLP

UPGMA

UT

UV
Soybean meal diet fed during Trial-2

Standard errors of the mean

Soy protein concentrate

Temperature gradient gel electrophoresis

Terminal restriction fragment length polymorphism

Unweighted pair group method algorithm

Universal target

Ultraviolet 


\section{Chapter 1 LITERATURE REVIEW}

\subsection{Aquaculture industry}

The Food and Agriculture Organization of the United Nations (FAO) reported that worldwide fisheries production exceeded 142 million tonnes of fish in 2008, an increase from 118 million tonnes in 1998 (FAO. 2010). This massive growth was possible because of industrialisation of the aquaculture sector. In contrast, world capture fish production in 2008 was estimated at 90 million tonnes and has been stable over the past 15 years (FAO. 2010). The FAO has estimated that growth in the aquaculture industry will continue, and outpace all other animal meat producing sectors due to its steady annual growth rate of $9 \%$ since 1970. The deficit in availability of fish and fish products that has been created due to the increase in consumption of seafood and stagnant capture fish production can be easily covered by the growth of the aquaculture industry. Globally, aquaculture products provide 1.5 billion people with $20 \%$ of their per capita intake of animal protein (FAO. 2010). In the coming years there will be a continuous growth in aquaculture production but it will not be at the same rate as seen in recent years owing to many factors such availability of sustainable diets, climate change, and economic challenges such as reducing use of fossil fuels and the corresponding burden on alternative energy resources (FAO. 2010). Almost forty-five million people are directly dependent on the aquaculture industry for their livelihood and income, with a $3.6 \%$ average rate of employment increase every year since 1980 (FAO. 2010). This development 
associated with the aquaculture industry affects 540 million people or $8 \%$ of the world's population who are directly or indirectly affiliated with this sector.

\subsection{The fish meal problem}

\subsubsection{Dependence on fish meal}

Fish meal (FM) and fish oil are major components of the fish feed that supports the ever growing aquaculture sector because of their ideal nutrient quality. FM is a prime, highly digestible source of protein, rich in essential amino acids such as lysine and methionine. Fish oil is a good source of eicosapentaenoic acid and docosahexaenoic acid, nutrients that are not found in plant ingredients and plant oil but are essential for growth of fish (Watanabe. 2002). The capture fish industry harvests fish like clupeids (Clupea sp., Ethmidium sp.), capelin (Mallotus villosus), and anchovy (Engraulidae sp.) for the direct production of FM and fish oil. The supply of FM and fish oil has been stagnant, making it more and more challenging to support the aquaculture industry. The biggest challenge that the industry is facing now is to find sustainable, economical and environmentally friendly substitutes for FM and fish oil. Growth in the aquaculture industry is a blessing but also a curse to wild fisheries stocks and natural ecological habitats. It is also a source of waste which further affects marine species and the environment. Dependence on FM in aqua-feeds further elevates the problem, with the potential to cause the collapse of naturally available world fish stocks (Naylor, et al. 2000). The industry 
perception of increase in aquaculture production compensating the wild fisheries production may not be entirely true as the production of farmed carnivorous species of fish in aquaculture requires 2.5 to 5 times more biomass than what is supplied by the farmed end product. Demand for FM and fish oil is growing immensely in the aquaculture industry but it is important to note that the swine and poultry sector are actually the biggest consumers of FM and fish oil (Naylor, et al. 2000) making it expensive and further constraining the growth of aquaculture industry.

\subsubsection{Alternatives for fishmeal}

The use of FM and fish oil will have to decrease in the long term for reasons such as diminishing global supplies of forage fish, increasing market price because of increase in global prices of energy, processing and shipping, and increasing pressure to substitute FM with other sustainable alternatives (Tacon, et al. 2008). A viable alternative for FM should be easily available, competitively priced, and easy to process, store and handle. Most importantly, it should have essential nutritional characteristics similar to FM and should not be linked to undesirable outcomes such as reduced growth, decreased health, and poor quality end product.

There are a few examples in the scientific literature supporting the idea of completely or substantially replacing FM in aqua feed with alternative protein sources (Regost, et al. 2003; Kaushik, et al. 2004). By-products from animal processing, plants, single cell products such as bacteria, micro-alage, yeast and 
protists, different types of zooplankton and insects have been used to replace FM (Tuominen, T. R. \& Esmark, M. 2003; Aas, et al. 2006; Olsen, et al. 2006; Olsen, et al. 2007). Planktons such as krill, copepods and micro-algae are high in protein and are a good source of omega 3 fatty acids, making them particularly good candidates for replacing FM (Cury, et al. 2000; Atalah, et al. 2007; Naylor, et al. 2009). Most of these studies suggest that replacement of FM with other marine and non-fish sources can be achieved without affecting fish health and product quality. However, exploitation of naturally available plankton stocks can have a negative effect on the ecological balance in higher trophic levels.

Compared to animal by-products, plant ingredients with their high protein content, essential amino acids, good palatability and high nutrient digestibility are better candidates to replace FM. These ingredients are available globally and have competitive prices. There are many studies focused on replacing FM with plant ingredients, but the findings are mainly based on the digestibility of the diets and usually the short duration of the study limits the application of the findings to understanding the effects of long-term usage of the se ingredients in aquatic diets. Nevertheless, many plant ingredients such as barley, wheat, corn, cottonseed, soybean, canola, peas have been used to replace FM and have been discussed by Gatlin et al. (Gatlin, et al. 2007).

Soybean, corn and wheat have been widely evaluated and used by producers and researchers all around the world for replacing FM in aqua feeds, with mixed results. Replacement of FM with soybean meal has been found to 
have negative effects on the health, feed efficiency and growth of fish (Ostaszewska, et al. 2005; Romarheim, et al. 2006), while others have reported benefits when these ingredients are included at low levels of 18 to $32 \%$ (Dabrowski, et al. 1989; Refstie, et al. 200; Morris, et al. 2005). On the other hand, soybean protein concentrate has been used successfully to replace FM at inclusion rates of up to $40 \%$ in aqua feeds (Heikkinen, et al. 2006).

Other plant ingredients that are commonly found in aqua feed are wheat and gluten meal. These ingredients are not only considered as replacements for FM but are used to reduce the use of soybean in aqua feed (Hardy. 2010). Corn meal gluten is another widely used plant ingredient in aqua feed but it is not a suitable candidate on its own unless supplemented with essential amino acids (Hardy. 2010).

\subsection{Challenges and limitations of including plant ingredients}

\subsubsection{Growth performance}

As mentioned earlier, FM and fish oil is rich in essential amino acids, which are required for optimum fish growth and are essential for fish health. FM is also a good source of energy. Reductions in the levels of FM and fish oil when replaced with alternative protein sources should not in any way reduce the amount of energy and nutrient content of the diet (Subcommittee on Fish Nutrition, National Research Council. 1993). However, palatability and digestibility of diets based on plant proteins can affect the growth and 
performance of fish. Feeding and digestibility experiments with diets based on plant ingredients with and without supplementation of essential amino acids showed that growth performance of fish was reduced in comparison with FM based diets (Gomes, et al. 1995; Espe, et al. 2006). This reduced growth performance was a result of reduction in feed intake. Atlantic cod (Gadus morhua) fed with plant based diets showed reduced appetite resulting in reduction in specific growth rate of the fish (Hansen, et al. 2007). Aqua feed formulated using plant ingredients contain higher fibre content (Robinson, et al. 1981), which reduces the digestibility of the feed (McGoogan, et al. 1996) since carnivorous fish are unable to easily digest carbohydrates as compared to other vertebrates and humans (Millward. 1989).

\subsubsection{Anti-nutritional factors}

It has been suggested that diets based on plant protein products can be sufficient for fish growth (Subcommittee on Fish Nutrition, National Research Council. 1993). However, these ingredients contain various anti-nutritional factors, which can negatively affect the growth and health of fish. Antinutritional factors can be defined as "naturally occurring toxins or antimetabolites, whose function in plants is as natural insecticides or, generally, as part of survival mechanis ms that prevent grazing and consumption of plants by insects and other herbivores" (Hendricks. 2002). Some plant associated antinutritional factors are trypsin inhibitors, hemagglutinins, gossypol, cyclopropenoic fatty acids, alkaloids and phytic acid (Subcommittee on Fish 
Nutrition, National Research Council. 1993; Refstie, et al. 1998). Phytic acid can bind phosphorous, which is an essential nutrient for fish and other nonruminants, making it less bio-available and digestible (Francis, et al. 2001). Phytic acid is just one example that shows that its presence in any diet can cause reduction in the nutritional value of the diet, resulting in a variety of physiological effects. These anti-nutritional factors can also cause reduction in protein intake and utilisation affecting normal growth (Krogdahl, et al. 1994; Vielma, et al. 2000). It has been suggested that anti-nutritional factors can be divided broadly into four groups based on their mechanism (Francis, et al. 2001): (1) factors that interfere with protein digestion and utilization, including protease inhibitors, tannins and lectins, (2) antivitamins, (3) factors that reduce mineral utilisation by making them unavailable or degraded, such as phytates, oxalates, glucosinolates, and gossypol, and (4) other toxic substances such as mycotoxins, mimosine, nitrates, alkaloids, saponins which are related to plant ingredients and have specific effects other than those described for the previous general categories. Anti-nutritional factors can be classified based on their heat stability. Factors that can withstand thermal processing are called heat stable factors, and those that are degraded by thermal processing are called heat labile factors.

Even though many studies have concentrated on the effects of feeding plant based diets to fish, the effects of anti-nutritional factors are not fully understood. Anti-nutritional factors as sociated with plant ingredient based diets reduce digestion and protein utilisation directly affecting the growth of 
salmonid fish (Krogdahl, et al. 2010). Researchers have also observed that inclusion of high levels of soybean in aqua feed is associated with distal gut inflammation and high numbers of intracellular vacuoles. Any diet related damage to the intestine can be characterised by markers like increase or decrease in numbers of mucus producing goblet cells, changes in mucosal folding and connective tissue, and increase in the number of inflammatory cells in the lamina propria (Baeverfjord, et al. 1996). Soybean-induced enteritis is a condition linked to extreme usage of soybean in fish diets, in which severe necrosis is seen in the intestine (Baeverfjord, et al. 1996). Nevertheless substitution of up to $75 \%$ of FM with plant ingredients in diets has been tried and has resulted in normal growth of Atlantic cod (Gadus morhua) with marginal effects (Olsen, et al. 2007).

Plant based diets fed to fish can affect the gastrointestinal microbiota (Ringo, et al. 1995; Ringo, et al. 1999; Olsen, et al. 2006; Ringo, et al. 2006a). These studies show that diet associated changes in the intestinal microbiota may be responsible for reduced growth. Recent studies investigating the effects of soybean meal based diets on the intestinal microbiota of Atlantic cod (Gadus morhua) and rainbow trout pointed to a probable role of intestinal microbiota in the aetiology of soybean meal induced enteritis (Heikkinen, et al. 2006; Ringo, et al. 2006c). In another study some information about altered intestinal microbial structure and its possible role in intestinal pathology in Arctic char (Salvelinus alpinus) fed with inulin can be found (Ringo, et al. 2006a). The mechanism through which intestinal inflammation is caused is not very clear, 
but evidence from studies of other mammals suggests that modification of the intestinal microbiota can disrupt signalling necessary for normal intestinal barrier function, resulting in a lack of recognition of commensal bacteria as non-pathogenic (Hooper, et al. 2001). It has been shown in intestinal bowel disease related studies that in germ free mice there is no intestinal inflammation suggesting a role of intestinal microbiota in the pathogensis of intestinal inflammation (Hanauer. 2004; Elson, et al. 2005). This information in relation to fish is not available. A better understanding of the aetiology of intestinal inflammation in fish and determination of the role of plant based ingredients in this process will require an improved understanding of the structure and function of the normal intestinal microbiota of fish.

\subsection{Intestinal microbiota of fish}

The microbial community of the intestine is more densely populated than the external environment, suggesting the provision of better ecological niches, favourable for the growth of these micro-organisms (Denev, et al. 2009). The cellular structure and physiology of digestion of the fish gastrointestinal tract are quite similar in some aspects to that of higher vertebrates so it might be assumed that the structure of the intestinal microbiota is also similar. However, the intestinal microbiota of fish is comparatively less dense and diverse than humans and other endotherms. The human intestinal microbiome is mainly composed of anaerobes which are present in numbers orders of magnitude higher than aerobes (Neish. 2009), whereas the fish intestinal microbiome is mainly 
comprised of aerobes and facultative anaerobes (Trust, et al. 1974). Similarly, when bacterial counts of human and other vertebrate intestinal microbiomes are compared to fish it is observed that the intestinal microbiota of fish is not as dense (Trust, et al. 1974; Trust. 1974; Neish. 2009). These microorganisms play an important role in the health and nutrition of the fish and many studies have been undertaken to characterize the structure of the fish intestinal microbiota.

\subsubsection{Beneficial role of intestinal microbiota}

The intestinal microbiota of fish plays important roles in metabolic as well as protective functions of the gastrointestinal tract (Denev, et al. 2009). The resident microbiota metabolises nutrients, has an antagonistic activity against known fish pathogens such as Vibrio spp. and competes with pathogenic bacteria and restricts their colonisation in the intestine (Denev, et al. 2009). Studies in zebra fish suggest that intestinal microbiota plays an important role in an immuno-physiological regulation between the host and the microbiota resulting in strengthening the immune system (Rawls, et al. 2004).

Growth of fish pathogens in the intestine of fish is countered by the antagonistic property of the resident intestinal microbiota (Saha, et al. 2006; Skrondenyte, et al. 2006; Sugita, et al. 2006). The beneficial intestinal microbiota of fish is known to compete with pathogenic bacteria for adhesion sites on the intestinal wall (Gomez, et al. 2008; Perez, et al. 2010) and available nutrients (Tinh, et al. 2008). It has been shown that pathogenic genera such as Aeromonas, Vibrio and Yersinia are restricted from adhering to the intestinal 
wall in the presence of a healthy intestinal microbiota. The adhesion of beneficial lactic acid bacteria to the intestinal wall also reduces the adhesion of pathogenic bacteria (Ringo, et al. 1995; Balcázar, et al. 2008).

The intestinal microbiota of fish is also known to perform enzymatic functions and produce cellulytic, lipolytic, amylolytic and proteolytic enzymes which help in digestion of simple and complex proteins, lipid, cellulose and chitin ingested in different feeds (Bairagi, et al. 2002; Sugita, et al. 2006). The enzyme producing function of the intestinal microbiota of fish has been of interest in the development and identification of prebiotics and probiotics (Bairagi, et al. 2002).

\subsubsection{Culture based characterisation of fish intestinal microbiota}

Culture based studies of the intestinal microbiota of fish have led to the understanding of the intestinal microbiota of different fish species being mainly composed of aerobes and facultative anaerobes (Cahill. 1990; Ringo, et al. 1995; Spanggaard, et al. 2000). Typically the intestinal microbiota of salmonid fish is composed of more of aerobic bacteria $\left(10^{8}\right.$ bacteria $\left.\mathrm{g}^{-1}\right)$ than anaerobic bacteria $\left(10^{5}\right.$ bacteria $\left.\mathrm{g}^{-1}\right)$ (Trust, et al. 1974; Trust, et al. 1974; Ringo, et al. 1995). Bacterial counts of the intestinal microbiota of Arctic char (Salvelinus alpinus) showed that approximately $10^{5}$ bacteria $\mathrm{g}^{-1}$ were present in the intestine and included predominantly Aeromonas, Acinetobacter, Cytophaga, Moraxella, Streptococcus, and Lactobacillus (Ringo, et al. 1994). 
Most studies characterising the intestinal microbiota using culture based techniques concentrate on aerobic culture methods to cultivate bacteria, leading to the possibly flawed conclusion that anaerobic bacteria play a minor role in the GI tract of fish (Spanggaard, et al. 2000). However, Spanggaard (2000) observed that both culture plate counts and microscopic estimates of aerobic bacteria were highly correlated, and concluded that anaerobic bacteria play a minor role as the counts were very low compared to aerobes and facultative anaerobes. As it is difficult to determine a bacterial species using microscopy these conclusions could be flawed. Most of the bacterial species identified in a study of obligate anaerobes from the intestines of gold fish (Carassius auratus) and rainbow trout were previously unidentified and thus the functions that they carried out were also not known (Trust, et al. 1979). Anaerobic bacteria from the intestinal microbiota of freshwater fish species were studied by Sakata et al. in 1980 (Sakata, et al. 1980). The main aim of this study was to compare the numbers of anaerobic and aerobic bacterial species in the intestines of freshwater fish. It was observed that there was a non-significant difference between the aerobic and anaerobic counts on culture media. Anaerobic jars were used to cultivate the anaerobic bacteria, which were not strictly anaerobic at the beginning but became anaerobic gradually, which might have affected the result. Clostridium, Porphorymonas, Fusobacterium and Bacteroides are some of the anaerobic bacteria genera isolated from the intestines of fish such as angelfish (Pterophyllum scalare), southern flounder (Paralichtys lethostigma) and oscars (Astronotus ocellatus) (Ramirez, et al. 2003). 
Culture-based studies are time consuming and logistically complex. Most studies conducted in the past employed only one nutrient medium at one temperature (usually $20^{\circ} \mathrm{C}$ ), and it has been shown that the total viable bacteria count can be different on different nutrient agars (Sugita, et al. 1988). It has been suggested that to characterize the intestinal microbiota thoroughly it is necessary to use multiple nutrient media, incubated at different temperatures so as to target the widest possible range of bacterial taxa (Ringo, et al. 1995). Despite the limitations of culture based techniques some findings, such as the fact that the microbime structure in different regions of the gastrointestinal tract is different, have formed the basis of our understanding of the structure of the fish intestinal microbiome. These studies have also shown that the bacterial species in different regions of the gut as well as the bacterial species adhered to the gut wall are different from the ones present in the intestinal contents of fish such as rainbow trout (Trust, et al. 1974)

\subsubsection{Culture independent characterisation of fish intestinal microbiota}

Limitations encountered with culture based methods can be overcome by molecular techniques, which are culture-independent and focus on characterization of bacterial community DNA. This fundamental idea has been used and exploited by many researchers in different ways. A major advantage of molecular methods is the ability to identify and quantify bacteria regardless of their culturability. These techniques have played an important role in understanding the changes taking place in the structure of intestinal microbiota

\section{3}


when perturbed by factors such as diet. Culture independent methods have been useful in characterising the intestinal microbiota of different species of fish and have supplemented the information gathered using culture based techniques (Hovda, et al. 2007; Denev, et al. 2009; Mansfield, et al. 2010; Merrifield, et al. 2010; Navarrete, et al. 2010).

PCR denaturing gradient gel electrophoresis (DGGE) is a rapid and easy to perform technique that has been the most widely used molecular fingerprinting techniques for characterization of fish intestinal microbiota (Spanggaard, et al. 2000; Huber, et al. 2004; Brunvold, et al. 2007; Hovda, et al. 2007; Zhou, et al. 2009). This technique is based on the 16S rRNA gene, found universally in all bacteria. DGGE is based on the principle that double-stranded DNA fragments with different nucleotide sequences will melt at different temperatures (exploited in temperature gradiet gel electrophoresis) or at different levels of chemical denaturants such as urea (Fischer, et al. 1983).

The first step in DGGE is to amplify all of the $16 \mathrm{~S}$ rRNA sequences present in a sample from a microbial community using universal PCR primers. A 30-50 base pair GC rich sequence ("GC clamp") is included at the 5' end of one of the primers to avoid complete dissociation of the double-stranded PCR product during electrophoresis (Sheffield, et al. 1989). PCR products are separated on a polyacrylamide gel containing a gradient of urea. DNA fragments with different sequences and GC contents have different melting behaviours in gels containing urea. Thus, as the PCR products migrate through the gel, encountering increasing concentrations of urea, the fragments become

\section{4}


increasingly denatured in a sequence-dependent manner, until their migration through the gel decreases or completely stops. Based on this principle if a genomic DNA sample consists of multiple bacterial species with different $16 \mathrm{~S}$ rRNA sequences, the result will be many bands on the gel, with the number of bands corresponding to the number of bacterial $16 \mathrm{~S}$ rRNA sequences present in that sample. The technique can be applied to multiple samples at the same time allowing the comparison of microbial profiles. To identify the different bacterial species in the sample, bands from the microbial profile can be recovered and sequenced. In this manner both cultivable and non-cultivable bacteria in the sample can be studied. Any PCR based technique poses the limitations of PCR bias. Preferential amplification occurring due to PCR bias can cause a problem for DGGE analysis. Problems such as chimera and hetroduplex formation can affect band resolution in DGGE profiles and are a major limitation to this technique (Liesack, et al. 1991; Kopczynski, et al. 1994). Length of fragment amplified in DGGE is also important since shorter fragments can decrease resolution and be an obstacle for reliable identification of microbial species. Bacterial species with similar GC content and sequences can be difficult to identify as they will have similar melting characteristics making it impossible to separate on the gel. Another problem inherent with PCR DGGE based on 16S rRNA gene is the possibility of multiple bands with a similar nearest neighbour resulting in over estimation of community diversity. (Nubel, et al. 1996). 
Other molecular based techniques such as temperature gradient gel electrophoresis (TGGE), terminal restriction fragment length polymorphism (TRFLP), and fluorescence in situ hybridization (FISH) have also been used to characterize the dominant bacterial components of the rainbow trout intestinal microbiota (Huber, et al. 2004; Sogin, et al. 2006; Mansfield, et al. 2010; Navarrete, et al. 2010; Fjellheim, et al. 2011).

The complete nucleotide sequence of $16 \mathrm{~S}$ rRNA gene of $E$. coli was determined in 1978 (Brosius, et al. 1978). Over the last two decades, sequencebased methods based on this gene target have become increasingly popular for the culture-independent characterization of complex microbial communities. More recently, the gene encoding the universal $60 \mathrm{kDa}$ chaperonin (cpn60) has been used in many sequence based microbial ecology studies as it has more discriminatory power than $16 \mathrm{~S}$ rRNA gene, making it more informative (Hill, et al. 2004). Cpn60 based methods involving the application of univeral cpn60 PCR primers have been used to characterise the intestinal and urogenital microbiota of various animals, including humans (Hill, et al. 2005a; Hill, et al. 2005b; Hill, et al. 2005b; Lukwinski, et al. 2006; Lukwinski, et al. 2006; Desai, et al. 2009; Desai, et al. 2009; Mansfield, et al. 2010; Schellenberg, et al. 2011 b). Since the cpn60 gene has a good discriminatory power and is usually present in a single copy in bacterial genomes, it is an ideal target for species specific PCR assays used for detection and quantification purposes (Dumonceaux, et al. 2006c). Quantitative real-time PCR (qRT-PCR) is a 
commonly used technique to quantify a specific bacterial species or a group of related taxa in complex samples.

Sequencing of libraries of cloned PCR products from universal gene targets such as $16 \mathrm{~S}$ rRNA or cpn60 has been used to characterise microbial communities associated with the fish intestine (Desai, et al. 2009; Caton, et al. 2011; Mark, et al. 2011). In general, this approach involves universal primer PCR of the target gene followed by ligation of the resulting PCR products into a suitable cloning vector for creation of a clone library in E. coli. Clones are randomly selected and sequenced, resulting in a collection of sequences representing the species present in the original sample. Individual sequences can be identified by comparison to a reference sequence database such as the RDP (Wang, et al. 2007) or cpnDB (Hill, et al. 2004). Clone library analysis is not a quantitative method since template DNA isolation and the production of PCR product with universal primers are subject to several biases that can affect the representation of individual species in the library (Bent, et al. 2008). However, it can give an idea about the dominant bacteria present in the environment. Characterisation of the intestinal microbiota using clone library analysis largely depends on the number of clones.

Recent developments in next generation high throughput sequencing technologies have made it possible to undertake much larger scale sequencing studies than were possible with clone library sequencing approaches (Margulies, et al. 2005). Next generation sequencing technologies such as the 454 pyrosequencing method (Schellenberg, et al. $2011 \mathrm{~b}$; Chaban, et al. 2012) have 
been adopted rapidly by microbial ecologists for their utility in creating detailed sequence based profiles of unprecedented depth. Recently, the cpn60 universal target was used as a suitable 454 pyrosequencing target to characterise microbial profiles (Schellenberg, et al. 2011b; Chaban, et al. 2012). Pyrosequencing does not rely on cloning PCR products, but depends on parallel sequencing of a large number of templates eliminating any potential biases associated with cloning. In short, PCR amplicons are bound to DNA capture beads in such a way that only one amplicon is attached per bead. Amplicons are subsequently amplified within individual reagent droplets (emulsion PCR) (Margulies, et al. 2005). These amplicons can then be sequenced using 454 pyrosequencing in which nucleotides are added to the reaction and base incorporation is detected by light emitting enzymatic reaction (Ronaghi, et al. 1998). The addition of short "barcode" sequences (multiplexing IDs, MID) to PCR primers used in pyrosequencing has made it possible to combine multiple samples in a single sequencing run, followed by bioinformatic separation of the sequence data derived from each sample. This modification makes it economically feasible to generate microbial profiles for a large number of samples in a single sequencing run. This approach has been used extensively to study animal and human microbiomes (Schellenberg, et al. 2009; Chaban, et al. 2012), including the zebrafish (Danio rerio) (Roeselers, et al. 2011). 


\subsubsection{Intestinal microbiota of salmonid fish}

Fish eggs hatch into larvae to become part of an ecosystem shared by different bacteria, some of which can be pathogenic (Egidius. 1987). Although historically the existence of a salmonid larval intestinal microbiota was questioned (Seki. 1969), it is now a well accepted fact that the intestine of a fish is colonized once it hatches from the egg (Hansen, et al. 1999).

Bacteria present in the surrounding environment have a big influence on the colonization of the salmonid fish intestine. Earlier it was suggested that the fish larvae intestine stays sterile until it consumes feed for the first time (Sugita, et al. 1988), however later it was shown that the bacteria present in the pond or incubator can get access to the gastrointestinal tract of the larvae and form the initial intestinal microbiome (Hansen, et al. 1999). A study evaluating the intestinal microbiota of gold fish (Carassius auratus) from egg hatching to the mature adult stage suggested a three stage development of the intestinal microbiota starting from an intestinal microbiota highly influenced by the diet, water and the microbiota initially associated with the eggs (Sugita, et al. 1988). This intestinal microbiota changes rapidly as a result of the development in the structure of the intestine in the larval fish. This shift of the intestinal microbiota is more prominent in fish such as turbot and Atlantic cod, which undergo extensive metamorphosis during the development from larvae to a juvenile stage but less prominent in salmonid fish because the intestinal structures of larval stage of salmonid fish are comparatively well developed (Al-Maghazachi, et al. 1984; Verreth, et al. 1992). In a study to evaluate the intestinal microbiota of 
masu salmon (Onchorhynchus masou) and chum salmon (Onchorhynchus keta) from egg to fry stage it was seen that at early developmental stage (fry) the bacterial load ranged from sterile to $10^{4} \mathrm{~g}^{-1}$ while it ranged from $10^{3}$ to $10^{7}$ bacteria $\mathrm{g}^{-1}$ in fingerlings (Yoshimizu, et al. 1980). It was also suggested that intestinal microbiota of fish at the fry stage was influenced by the water and the diet and consisted mostly of species like Coryneforms and Pseudomonas. With development from young to an adult fry this intestinal microbiota gets shifted to Achromobacter spp., Aeromonas spp. and Pseudomonas spp. These bacterial species were found to be absent or present at lower numbers in the intestinal microbiota of salmon at the fingerling stage (Yoshimizu, et al. 1980).

It has been suggested that Lactobacillus spp. are a major component of the intestinal microbiome of salmonid fish (Ringo, et al. 1995). These lactic acid bacteria play an important role in protecting fish during the developmental stages from immature larva to adult by producing growth inhibiting factors which suppress the growth of harmful bacteria such as Vibrio spp.. Salmonid fish live in both freshwater and marine environments and it has been suggested that the intestinal microbiomes of freshwater and marine salmonids are distinct in composition. A contrast is seen in the intestinal microbiota of salmonid fish and other fish from seawater where the intestinal microbiota is composed of obligate anaerobic bacteria species such as Clostridium and Bacteroides spp. (Ringo, et al. 1995). In the case of freshwater Salmonid fish the intestinal microbiota is mainly composed of bacteria from the family Enterobacteriaceae, Aeromonas spp., Plesiomonas and obligate anaerobic bacteria of the genera 
Fusobacterium and Eubacterium (Trust, et al. 1979; Sakata, et al. 1980; Huber, et al. 2004; Kapetanovic, et al. 2005; Hovda, et al. 2007). The intestinal microbiota of salmonid fish changes with migration from seawater to freshwater owing to changes in the water salinity (Yoshimizu, et al. 1976a; Yoshimizu, et al. 1976b). It is well documented that the intestinal microbiota of salmonids, like other fish families, is comprised of aerobic, facultative and obligate anaerobic bacteria (Trust, et al. 1979; Sakata, et al. 1980; Sugita, et al. 1985; MacFarlane, et al. 1986).

When the intestinal microbiomes of free living and farmed Arctic charr were compared it was seen that the intestinal microbiota of farm raised charr was comparatively more diverse. The intestinal microbiota of farmed as well as free living fish had common bacterial genera such as Acinetobactor, Aeromonas, Flavobacterium, Vibrio, Pseudomonas, Lactobacillus, Micrococcus and Streptococcus but the intestinal microbiota of farmed Arctic charr also included additional bacterial genera such as Kurthia, Agrobacterium, Brevibacterium, Streptococcus, Microbacterium, Arthobacterium, and Alcaligenes (Ringo, et al. 1994).

Distinct populations of bacteria have been recognized colonizing the mucosa and lumen (contents) of the intestines of fish and other vertebrates (Trust, et al. 1974; Trust, et al. 1979; Huber, et al. 2004; Kim, et al. 2007; Navarrete, et al. 2009; Navarrete, et al. 2010; Blaut. 2011). Studies evaluating the number of total bacteria present in the gastrointestinal tract of salmonid fish have had contrasting results. Studies looking at the intestinal microbiota of 
rainbow trout suggest that the number of aerobic heterotrophic bacteria decrease from the proximal to the distal intestine while other studies looking at the intestinal microbiota of Arctic charr suggest the opposite trend (Austin, et al. 1988; Ringo. 1994). Results of studies of yellow tail (Seriola quinqueradiata) and grass carp (Ctenopharyngodon idella) support the observation of increasing numbers of bacteria from the proximal to distal intestine (Trust, et al. 1979; Sakata, et al. 1980).

Various studies have been done to study the effects of plant protein based diets on the growth of fish (Aas, et al. 2006; Borgeson, et al. 2006; Espe, et al. 2006) but very few studies have been done to study the effects of these diets on the intestinal microbial community (Borgeson, et al. 2006; Heikkinen, et al. 2006; Ringo, et al. 2006b; Bakke-McKellep, et al. 2007). These studies have reported presence of different species in the intestinal microbiota of fish fed with plant ingredients and FM based diets, but there is consensus that intestinal microbiota of fish fed with plant based diets is more diverse and richer as compared to their FM fed counterparts (Ringo, et al. 2006b; Silva, et al. 2011). These studies have been based manily on culture based techniques used to isolate intestinal bacterial and then characterising them by biochemical test or PCR sequencing. There are no reports of large scale molecular studies aimed at characterising the intestinal microbiota and investigating the changes taking place in the intestinal microbiota in association with different plant based diets. 


\section{Chapter 2 Hypothesis and objectives:}

This study was designed to test the hypothesis that the structure of the intestinal microbiota is altered when rainbow trout are fed with different plant based diets. Testing this hypothesis will contribute to the long term goal of understanding the microbial ecology of the fish intestine and the relationship between diet, intestinal microbiota and intestinal health.

The objectives of the study are:

1) To apply cpn60-based culture independent methods to describe the composition of the intestinal microbiome of farmed trout.

2) To determine the structure of the intestinal microbiome of rainbow trout fed with different diets formulated using plant ingredients (soybean, canola and peas) at two different processing levels (meal and protein concentrate).

3) To determine if the effects of plant based diets on the intestinal microbiome of fish are reproducible.

4) To evaluate the extent to which bacterial strains are shared by fish reared in a recirculating aquaculture facility, using Carnobacterium maltaromaticum as an example. 


\section{Chapter 3 Characterization of rainbow trout (Oncorhynchus mykiss) intestinal microbiota and inflammatory marker gene expression in a recirculating aquaculture system}

Chapter 3 is a part of a published multi-disciplinary study. It is reproduced here with permission of the copyright owner (Elsevier).

Mansfield GS, Desai AR, Nilson SA, Van Kessel AG, Drew MD, Hill JE (2010). Characterization of rainbow trout (Oncorhynchus mykiss) intestinal microbiota and inflammatory marker gene expression in a recirculating aquaculture system. Aquaculture 307:95-104.

Author contributions

All authors participated in the design of the experiments and contributed to writing of the manuscript. Nilson was responsible for diet formulation and collecting and analysing digestibility data. Inflammatory marker gene expression analysis was conducted by Mansfield. The author of this thesis contributed to diet making, fish husbandry and sample collection, and was responsible for cpn60 clone library generation and anlysis, and qRT-PCR of bacterial targets. 


\subsection{Abstract}

Intestinal microbiota and host inflammatory marker gene expression were characterized in rainbow trout (Oncorhynchus mykiss) during a feeding trial designed to determine the digestibility of soybean meal and fish meal. The trial was conducted in a freshwater, biofiltered recirculating aquaculture system in Saskatoon, Canada. Intestinal contents and tissue were collected from nine fish on each of the two experimental diets and a synthetic, casein-based reference diet. Total DNA from contents of each group was pooled to create template for PCR and construction of libraries of cloned cpn60 universal target sequences. A total of 3357 sequences were produced. The most frequently observed sequences were similar to Firmicutes (particularly Carnobacterium maltaromaticum) and Gamma Proteobacteria, including enterobacteria genera Hafnia, Pseudomonas and Aeromonas. The fish meal diet associated microbiota was balanced between Gamma Proteobacteria and Firmicutes while the soybean meal diet associated microbiota was less diverse and dominated by $C$. maltaromaticum. Species-specific qPCR of several bacterial species revealed a large amount of variation in target abundance between individual fish. Analys is of inflammatory marker genes including proliferating cell nuclear antigen (PCNA), immunoglobulin M (IgM), and interleukin-1 beta (IL-1 $\beta$ ) showed a significantly higher level of PCNA associated with the reference diet and a nonsignificant trend of elevated IL-1 $\beta$, suggesting that the semi-purified reference diet may be associated with sub-acute intestinal damage. A high level of variation between individual animals was also observed in the gene expression 
analysis. An examination of correlations within the combined results support the model that diet effects on the fish intestine include indirect effects mediated through modifications of the intestinal microbiota. These results illustrate the benefits of collecting experimental data from individual fish to increase the analytical power of results. 


\subsection{Introduction}

The complex microbial community of the intestine (intestinal microbiota) of terrestrial animals plays a critical role in the digestion of food, pathogen exclusion and the development and maturation of the immune system. In fish, the intestinal microbiota is not as dense (viable counts approximately $10^{8} \mathrm{cfu}^{-1}$ (Kim, et al. 2007; Navarrete, et al. 2010) but is presumed to play a similar role in the health and growth of the host. Recent research focused on the potential replacement of fish meal in aqua feeds with plant-derived proteins has led to the observation of pathological changes and inflammation in the intestinal mucosa which are thought to be either a direct effect of anti-nutritional factors on the gut epithelium and/or the result of diet-induced changes in the microbiota structure and function (Bakke-McKellep, et al. 2000; Krogdahl, et al. 2003; Sanden, et al. 2005; Bakke-McKellep, et al. 2007). Given the interaction between host, microbiota and diet, understanding these relationships is critical to development and evaluation of novel diets and maximizing fish health and welfare.

Historically, our understanding of the composition of the intestinal microbiota was limited by reliance on culture-based methods where only those organisms that could be readily cultivated in the laboratory could be identified. More recently, culture-independent, molecular methods have been applied to the study of intestinal microbiology, complementing culture-based studies and resulting in an increased appreciation of the complexity of the community. The predominant molecular methods employed in studying fish intestinal microbiota 
have been fingerprint-style methods, particularly denaturing gradient gel electrophoresis (DGGE) on microbial community samples or restriction fragment length polymorphism (RFLP) analys is for identification of isolates. Although DNA sequencing has been widely employed in the identification of cultured bacterial isolates from the intestinal environment, very few sequencebased studies of the entire intestinal microbiota have been conducted and this work has been done almost exclusively based on sequencing of cloned $16 \mathrm{~S}$ rRNA genes (Holben, et al. 2002; Kim, et al. 2007).

One particularly important outcome of work to date has been the description of variation in microbial composition and population density between fish species and among individuals of the same species, as well as variation associated with environmental factors such as management style, temperature and salinity (reviewed in (Cahill. 1990) and (Ringo, et al. 1995)). This is consistent with studies of other animal species where the microbiota is observed to change in structure with development and environmental changes. These observations emphasize the necessity of having a thorough understanding of the particular experimental system in which nutrition and growth studies take place, including quantifying normal variation in microbiota within the system (within and between animals), making it possible to recognize biologically significant deviations. Currently employed molecular methods have been of limited use since most do not provide sufficient detail or resolution to adequately describe differences or recognize shifts in microbiota composition. 
The cpn60 gene (encoding the universal $60 \mathrm{kDa}$ chaperonin) has been established as a target for sequence-based microbial ecology studies and has been demonstrated to be generally more informative than $16 \mathrm{~S}$ rRNA, providing better discrimination between closely related organisms (Hill, et al. 2004). A 552-567 bp region of cpn60, corresponding to nucleotides 274-828 of the E. coli cpn60 gene, can be amplified with universal, degenerate PCR primers (Hill, et al. 2006). To date, cpn60 sequence-based methods have been applied to the study of intestinal and urogenital microbiota of a variety of terrestrial animals, including humans (Hill, et al. 2005a; Hill, et al. 2005b; Dumonceaux, et al. 2006a; Lukwinski, et al. 2006; Desai, et al. 2009). The discriminating power of the cpn60 universal target sequence also makes it an ideal target for the development of species-specific PCR assays for the detection and quantification of bacteria in complex samples (Dumonceaux, et al. 2006c).

The challenges in characterization of the intestinal microbiota also apply to investigations of host response, where natural variation in marker gene expression must be understood before conclusions can be drawn regarding the effects of experimental changes in diet or environment. Variation in host gene expression, even for so-called housekeeping genes, is widely experienced but rarely reported (Juul-Madsen, et al. 1992; Nath, et al. 2006). As a result, it is necessary to examine a number of gene markers associated with any particular physiological pathway and/or systematically identify the markers that are most informative. 
The Prairie Aquaculture Research Centre in Saskatoon, Canada is a biologically filtered, recirculating, freshwater system using a de-chlorinated municipal water supply and housing rainbow trout. The objective of our study was to take advantage of an ongoing digestibility trial to characterize the intestinal contents microbiota of rainbow trout on three different diets and to measure host inflammatory marker gene expression in the intestines of fish in this research facility. In addition, we applied species-specific quantitative PCR to intestinal contents samples from individual fish in order to investigate the level of animal to animal variation within and between diet groups. The results of this study add to our understanding of the intestinal microbiota of rainbow trout and further describe the extent of individual to individual variation in fish that should be an important consideration in experimental design. These results form the foundation for future trials and experiments aimed at understanding the interactions between host, microbiota and diet. 


\subsection{Material and Methods}

\subsubsection{Ingredients and diets}

The reference diet and two experimental diets, which contained either fish meal or soybean meal at an inclusion level of $30 \%$ are described in Table 3.1 . The reference diet was formulated based on the semi-purified reference diet reported by (Cho, et al. 1985) and modified by the addition of Celite 545 highpurity flux-calcined diatomaceous earth (Celite Co., World Minerals Co., Lompoc, CA, USA) partially in place of alpha cellulose, a non-nutritive filler (NRC, 1993) (NRC (National Research Council). 1993), as a non-absorbable indicator for indirect digestibility analys is. Experimental diets were made by combining the reference mash with either test ingredient at a mass ratio of $7: 3$. Diets were cold pelleted through a $3 \mathrm{~mm}$ die on a Hobart mixer (Hobart Corp., Troy, $\mathrm{OH}$, USA), dried in a forced air oven for $12 \mathrm{~h}$ at $55^{\circ} \mathrm{C}$, then chopped and screened to form pellets of uniform size. 
Table 3.1 Ingredient composition and nutrient analysis of diets (g kg-1).

\begin{tabular}{|c|c|c|c|}
\hline Ingredient & Purified & Fish meal & $\begin{array}{l}\text { Soybean } \\
\text { meal }\end{array}$ \\
\hline Casein $^{a}$ & 400 & 280 & 280 \\
\hline Fish meal $^{\mathrm{b}}$ & 0 & 300 & 0 \\
\hline Soybean meal ${ }^{\mathrm{c}}$ & 0 & 0 & 300 \\
\hline Fish oil ${ }^{\mathrm{d}}$ & 150 & 105 & 105 \\
\hline Starch ${ }^{e}$ & 115 & 80.5 & 80.5 \\
\hline Dextrin $^{f}$ & 90 & 63 & 63 \\
\hline Mineral premix ${ }^{\mathrm{g}}$ & 80 & 56 & 56 \\
\hline$D-g l u \cos \mathrm{e}^{\mathrm{h}}$ & 50 & 35 & 35 \\
\hline Gelatin ${ }^{\mathrm{i}}$ & 40 & 28 & 28 \\
\hline Vitamin premi $^{\mathrm{j}}$ & 29.5 & 20.65 & 20.65 \\
\hline Alpha-cellu los $\mathrm{e}^{\mathrm{k}}$ & 20 & 14 & 14 \\
\hline Diatomaceous earth ${ }^{1}$ & 10 & 7 & 7 \\
\hline $\mathrm{L}-\arg$ inin $\mathrm{e}^{\mathrm{m}}$ & 10 & 7 & 7 \\
\hline DL-meth ion in $\mathrm{e}^{\mathrm{n}}$ & 5 & 3.5 & 3.5 \\
\hline Vitamin $\mathrm{C}^{\mathrm{O}}$ & 0.5 & 0.35 & 0.35 \\
\hline \multicolumn{4}{|l|}{ Dietarycomponent $(\mathrm{g} / \mathrm{kg})^{\mathrm{p}}$} \\
\hline Crude Protein & 483 & 564 & 512 \\
\hline Gross Energy (kcal/kg) & 5470 & 5470 & 5400 \\
\hline Ash & 32 & 63 & 31 \\
\hline Acid Ether Extract & 160 & 156 & 133 \\
\hline Starch & 115 & 81 & 81 \\
\hline Neutral Detergent Fibre & 20 & 07 & 60 \\
\hline Acid Detergent Fibre & 20 & 07 & 92 \\
\hline
\end{tabular}

${ }^{a}$ Casein from bovine milk, technical grade; Sigma-Aldrich Inc., St. Louis, MO, USA.

${ }^{b}$ Nova Scotia herring meal; Shur-Gain Aquaculture, Truro, NS, Canada.

${ }^{c}$ Soybean meal; Federated Cooperatives Limited, Saskatoon, SK, Canada.

${ }^{d}$ Danish Fish Oil; FF of Denmark, Skagen, Denmark.

e Starch-Corn; USB Corporation, Cleveland, OH, USA.

${ }^{\mathrm{f}}$ Dextrin; USB Corporation, Cleveland, OH, USA.

g Mineral premix, commercial (EWOS FISH MINERAL PX\#2, Surrey, BC; closed formulation), formulated to meet the requirements of juvenile rainbow trout; BASF Canada, Abbotsford, BC, Canada.

${ }^{\mathrm{h}}$ Dextrose, anhydrous; Sigma-A ldrich Canada, Ltd., Oakville, ON, Canada.

i 175 Bloom gelatin; Dawn Food Products (Canada), Ltd. Saskatoon, SK, Canada.

j Vitamin premix, commercial (EW OS FISH-STR VIT PX, Surrey, BC; closed formulation), formulated to meet the requirements of juvenile rainbow trout; BASF Canada, Surrey, BC, Canada.

${ }^{k}$ Solka-floc ${ }^{\circledR}, 200$ FCC; International fiber corporation, North Tonawanda, NY, USA.

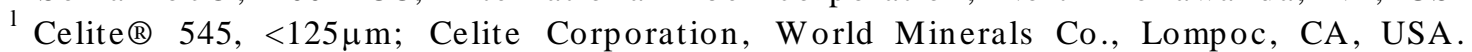

${ }^{\mathrm{m}}$ L-arginine monohydrochloride, not synthetic; Sigma-Aldrich, St. Louis, MO, USA.

${ }^{\mathrm{n}}$ DL-methionine, feed grade. Degussa Corporation, Theodore, AL, USA.

${ }^{\circ}$ Ascorbic acid, pharmaceutical grade; NOW Foods, Bloomingdale, IL, USA.

${ }^{\mathrm{p}}$ All dietary components were analyzed as described in the materials and methods except for starch and acid and neutral detergent fibre for the purified and fish meal diets which were calculated. 


\subsubsection{Fish husbandry and digestibility trial design}

The trial was conducted at the Prairie Aquaculture Research Centre (University of Saskatchewan, Saskatoon, SK), a biologically filtered, recirculating aquaculture system. The fish were triploid female rainbow trout (Oncorhynchus mykiss), acquired from Wild West Steelhead (Lucky Lake, SK) weighing $1.56 \mathrm{~kg} \pm 0.09 \mathrm{~kg}$ at the start of the experiment. They were housed in $120 \mathrm{~L}$ tanks with a water temperature of $15 \pm 1{ }^{\circ} \mathrm{C}$ and a photoperiod of $14 \mathrm{~h}$ light:10 h dark. Environmental and water quality indicators were closely monitored over the course of the experiment. The guidelines set by the Canadian Council on Animal Care (CCAC, 2005) were followed in the maintenance of all fish for the duration of this trial.

All fish were hand-fed twice daily to apparent satiation. A commercial fish meal diet was fed for two weeks prior to the experiment to acclimate the fish to their environment. Upon commencement of the trial, nine $120 \mathrm{~L}$ tanks were used, with three replicates per treatment and ten fish per tank $(691.2 \mathrm{~g}$ average weight). The reference, fish meal and soybean meal diets were randomly assigned to the tanks and fed for an eight week period.

Following the feeding period, three fish were taken from each tank (a total of nine fish per diet collected 2-5 hours after the last feeding) and euthanized by a sharp blow to the cranium prior to dissection. Sub-sampling was conducted with the aim of collecting average-sized animals from each tank, avoiding extremes in body size.

\section{3}




\subsubsection{Digestibility analysis}

Following six days of acclimation to the experimental diets, fecal material was collected daily over a three-week period using a settling column(Hajen, et al. 1993). Feces were centrifuged at $5000 \mathrm{x} \mathrm{g}$ for $15 \mathrm{~min}$, frozen, and freezedried. The freeze-dried feces were ground to pass through a $1 \mathrm{~mm}$ screen (Retsch mill, Brinkmann Corp., Newtown, PA). Analysis of the diets and fecal material included moisture (AOAC, 1990, method no. 934.01), energy (oxygen bomb calorimetry; Parr Adiabatic Calorimeter, Model 1200), crude protein, dry matter (100-moisture), acid ether extract (AOAC, 1995, method no. 954.02), acid detergent fibre (AOAC 973.18), neutral detergent fibre (AOAC 2002.04) and acid insoluble ash (Vogtmann, et al. 1975). The combustion method (AOAC, 1995) was used to determine nitrogen content, which was then multiplied by 6.25 in order to estimate protein. Apparent digestibility coefficients (ADC) for fish meal and soybean meal were calculated using the equations described by (Forster. 1999).

For the determination of acid insoluble ash (AIA) content, samples were charred at $250{ }^{\circ} \mathrm{C}$ for $18 \mathrm{~h}$, then gradually heated to $500{ }^{\circ} \mathrm{C}$ over a period of $3 \mathrm{~h}$ and held at that temperature to ash for $48 \mathrm{~h}$. Four $\mathrm{ml}$ of $4 \mathrm{~N} \mathrm{HCl}$ was added to the ashed samples, which were then heated for a minimum of one hour at $120{ }^{\circ} \mathrm{C}$, then centrifuged at $3000 \times \mathrm{g}$ for $10 \mathrm{~min}$. The supernatant was aspirated and 5 $\mathrm{ml}$ of water was added to rinse the pellet. The sample was vortexed, centrifuged, aspirated, and then rinsed a second time. The remaining pellets were dried at 80 ${ }^{\circ} \mathrm{C}$ for $14 \mathrm{~h}$ and then ashed at $500{ }^{\circ} \mathrm{C}$ for $24 \mathrm{~h}$. 


\subsubsection{Intestinal contents and tissue collection}

Intestines were subdivided into two sections: mid gut and distal gut. Mid gut was defined as the section from the last pyloric cecum to the beginning of the distal gut (visual increase in diameter or $50 \%$ of the distance from last pyloric ceca to the vent). Distal gut was defined as the section from the end of the mid gut to the vent. Digesta samples were collected aseptically from each intestinal section by squeezing the contents out of the section into individual sterile containers and stored on ice prior to transfer into storage at $-80{ }^{\circ} \mathrm{C}$. Remaining tissues were snap-frozen in liquid nitrogen and stored at $-80{ }^{\circ} \mathrm{C}$ prior to RNA extraction.

\subsubsection{Nucleic acid extraction}

Total RNA was extracted from 30-50 mg of ground intestinal tissue using TRI Reagent Solution (Applied Biosystems / Ambion, Inc., Austin, TX). Extracted RNA was subjected to an RNase-free DNase digestion using TURBO DNA-free (Applied Biosystems / Ambion, Inc., Austin, TX). The DNA-free RN A extracts were stored at $-80{ }^{\circ} \mathrm{C}$. The RNA content of the extracted samples was assessed using a Quant-iT RiboGreen kit (Invitrogen Canada Inc., Burlington, ON). Selected samples were also tested using an Agilent 2100 Bioanalyzer (Agilent Technologies, Inc., Santa Clara, CA) in order to verify the RNA extracts were of a high quality. One $\mu \mathrm{g}$ of RNA was then used to generate first strand cDNA using a High Capacity cDNA Reverse Transcription Kit (Applied Biosystems, Inc., Foster City, CA). 
Total genomic DNA was extracted from distal gut contents of individual fish using the QIAamp DNA Stool Mini Kit (Qiagen Inc., Mississauga, ON). Equal volumes of individual DNA extracts were pooled to create three pooled samples for PCR and cpn60 library construction.

\subsection{6 cpn60 clone libraries}

Libraries of cloned cpn60 universal target regions were constructed in cloning vector pGEM T Easy (Promega Co., Madison, WI, USA) as described previously(Desai, et al. 2009). Libraries were generated from pooled intestinal contents of nine fish for each of the three diets: reference control $(\mathrm{CN})$, fish meal (FM) and soybean meal (SBM). A total of 1,248 colonies were picked randomly from each of the three libraries.

Clones were sequenced using the $\mathrm{T} 7$ sequencing primer at the National Research Council Plant Biotechnology Institute (Saskatoon, SK) and raw sequence data was processed as previously described (Desai, et al. 2009). Representatives of all unique cpn60 sequences were deposited in GenBank (Accessions GU726297-GU726329, GU903291) and the cpn60 reference database (cpnDB, http://www.cpndb.ca) (Hill, et al. 2004). Nearest neighbors of library sequences were identified through a FASTA search of the cpnDB reference data collection (Pearson, et al. 1988). Phylogenetic trees were constructed using the PHYLIP software package and viewed using Treeview (Page. 1996). 


\subsubsection{Quantitative real-time PCR}

PCR primers used in quantitative PCR (qPCR) assays are listed in Table 3.2. Primers were designed using Beacon Designer 4.0 (PREMIER Biosoft International, Palo Alto, CA, USA). Primers for the amplification of $O$. mykiss genes were based on published sequences for beta-actin ( $\beta$-act, Accession AF157514), immunoglobulin M (IgM, X83372), interleukin-1 beta (IL-1 $\beta$, AJ004821), and proliferating cell nuclear antigen (PCNA, CA359986). $\beta$-act was included as an internal control for normalization of gene expression data. For construction of plasmid standard curves, PCR product from each primer set was ligated into cloning vector pGEM T Easy (Promega Co., Madison, WI) and sequenced to confirm the identity of the insert. For detection of bacterial cpn60 sequences, primer design was based on cloned sequences from the distal gut cpn60 clone libraries. For all qPCR primer pairs, optimal annealing temperatures were determined by examining the results of an annealing temperature gradient. Primer specificity was evaluated by comparison of primer sequences to the Genbank sequence database or cpnDB using BLASTn configured for short, nearly exact matches (Altschul, et al. 1990).

For conventional PCR of $O$. mykiss gene targets, reaction mixtures contained $1 \times$ PCR buffer, $1.5 \mathrm{mM} \mathrm{MgCl}_{2}, 200 \mathrm{nM}$ dNTPs, $500 \mathrm{nM}$ of each primer, $0.2 \mu 1$ of Taq polymerase, template cDNA isolated from intestinal samples in a final volume of $50 \mu 1$. PCR reactions were performed on an iCycler thermal cycler (Biorad Laboratories, Mississauga, ON). Cycling parameters were $95{ }^{\circ} \mathrm{C}$ for $5 \mathrm{~min}$, followed by 30 cycles of $1.5 \mathrm{~min}$ consisting of $30 \mathrm{~s}$ at 
$95{ }^{\circ} \mathrm{C}, 30$ s at $52-60{ }^{\circ} \mathrm{C}$, and 30 s at $72{ }^{\circ} \mathrm{C}$. For qPCR, reaction mixtures contained Platinum S YBR Green qPCR SuperMix-UDG (Invitrogen Canada Inc., Burlington, ON), $500 \mathrm{nM}$ of each primer, $2 \mu 1$ of template cDNA isolated from intestinal samples in a final volume of $25 \mu 1$. Cycling parameters were: $52{ }^{\circ} \mathrm{C}$ for $2 \mathrm{~min}$, followed by $95{ }^{\circ} \mathrm{C}$ for $3 \mathrm{~min}$, followed by 40 cycles of $40 \mathrm{~s}$ at $95{ }^{\circ} \mathrm{C}$, $40 \mathrm{~s}$ at the annealing temperature, and $40 \mathrm{~s}$ at $72{ }^{\circ} \mathrm{C}$, followed by a dissociation curve stepping from $65{ }^{\circ} \mathrm{C}$ to $95{ }^{\circ} \mathrm{C}$ in $0.5{ }^{\circ} \mathrm{C}$ increments. Quantification was accomplished through the use of a standard curve derived from a ten-fold dilution series of plasmid DNA containing the appropriate target sequence.

For qPCR detection of bacterial cpn60 sequences reaction mixtures contained 1× SYBR Green Supermix (Biorad Laboratories, Mississauga, ON), $400 \mathrm{nM}$ of each primer, $2 \mu 1$ of template DNA extract in a final volume of $25 \mu 1$. Cycling parameters were: $95{ }^{\circ} \mathrm{C}$ for $3 \mathrm{~min}$, followed by 40 cycles of $10 \mathrm{~s}$ at $95{ }^{\circ} \mathrm{C}, 30$ s at $64{ }^{\circ} \mathrm{C}$, and $30 \mathrm{~s}$ at $72{ }^{\circ} \mathrm{C}$, followed by a dissociation curve stepping from $65{ }^{\circ} \mathrm{C}$ to $95{ }^{\circ} \mathrm{C}$ in $0.5{ }^{\circ} \mathrm{C}$ increments. Reactions were run in duplicate on a Biorad MyiQ thermocycler (Biorad Laboratories, Mississauga, ON). Determination of target copy number was based on a standard curve generated from a tenfold dilution series of the target sequence clone. 


\section{Table 3.2 qPCR assays for intestinal gene expression analysis and quantification of bacteria.}

\begin{tabular}{|c|c|c|c|c|}
\hline Target & Primer sequences $\left(5^{\prime}-3^{\prime}\right)$ & $\begin{array}{l}\text { Sequence } \\
\text { frequency } \\
\text { (CN:FM:SB) }\end{array}$ & $\begin{array}{l}\text { Annealing } \\
\text { temp }\left({ }^{\circ} \mathrm{C}\right)\end{array}$ & $\begin{array}{l}\text { Amplicon } \\
\text { size (bp) }\end{array}$ \\
\hline O. mykiss $\beta$-act & $\begin{array}{l}\text { GCGACCT CACAGACT ACC } \\
\text { ACAGT CCCAT T GCT CCAGT CC }\end{array}$ & & 52 & 273 \\
\hline O. mykiss IgM & $\begin{array}{l}\text { CACT T CAT CAGAT GGT CCA GT CC } \\
\text { ACAGT CCCAT T GCT CCAGT CC }\end{array}$ & & 53 & 243 \\
\hline O. mykiss PCNA & $\begin{array}{l}\text { T GT GACCGCAACCT CGCAAT GG } \\
\text { CACGGCAGAT ACGGGCAAACT CC }\end{array}$ & & 60 & 265 \\
\hline Carnobacterium maltaromaticum & $\begin{array}{l}\text { GCAAT T T GACCGT GGT T ACC } \\
\text { T T AAT AATGGACCT T GT T GG }\end{array}$ & $1032: 537: 1148$ & 64 & 167 \\
\hline Hafnia alvae & $\begin{array}{l}\text { T ACT GGCT CAGGCT AT CG } \\
\text { CGGT T T CGT CGGAGT T AG }\end{array}$ & $0: 365: 0$ & 64 & 192 \\
\hline Leuconostoc citreum & $\begin{array}{l}\text { GAT CGAGGT T ACAT GT CACAGT AC } \\
\text { CAACAACAGCT T GT AACACAGG }\end{array}$ & $0: 23: 0$ & 64 & 139 \\
\hline
\end{tabular}




\subsubsection{Statistical analysis}

Analysis of digestibility and gene expression results was performed using the General Linear Model procedure of SPSS 14.0 (SPSS Inc., Chicago, IL, USA). Differences between means were determined using the Ryan-EinotGabriel-Welsh F test for digestibility data and Tukey's HSD test for gene expression data. Significance of results were determined when $P<0.05$, nonsignificant trends were determined when $0.10<P>0.05$. Two-tailed Pearson correlation coefficients were calculated between diets, intestinal gene expression and intestinal bacterial populations. Correlations were considered significant when $P<0.05$. 


\subsection{Results}

\subsubsection{Digestibility}

The fish meal diet had significantly $(P<0.05)$ higher dry matter and gross energy ADCs than the reference and soybean meal diets. Dry matter ADCs were 0.82 for the fish meal diet and 0.77 for both reference and soybean meal diets. Gross energy ADCs were 0.88 for the fish meal diet, 0.82 for the soybean meal diet and 0.81 for the reference diet. Crude protein ADCs were not significantly different between the three treatments. The dry matter and gross energy ADCs for fish meal were 0.95 and 1.07, respectively, which were significantly higher than the dry matter (0.07) and gross energy (0.83) ADCs for soybean meal (Table 3.3). The ADC of energy for the fishmeal was over 1.00 . This might have been due to variability in the measurement of energy in the diets and faeces. Using these values in the formula for calculating the energy ADC in the formula may have resulted in the value reported in Table 3.1. 
Table 3.3 Apparent digestibility coefficients for experimental diets and ingredients in rainbow trout (dry matter basis).

\begin{tabular}{lllll}
\hline & Dry matter & Crude protein & Gross energy & Ash \\
\hline Diets & & & & \\
Reference & $0.77^{\mathrm{a}}$ & 0.92 & $0.81^{\mathrm{a}}$ & $0.47^{\mathrm{b}}$ \\
Fish meal & $0.82^{\mathrm{b}}$ & 0.95 & $0.88^{\mathrm{b}}$ & $0.33^{\mathrm{a}}$ \\
Soybean meal & $0.77^{\mathrm{a}}$ & 0.93 & $0.82^{\mathrm{a}}$ & $0.33^{\mathrm{a}}$ \\
SEM & 0.000 & 0.018 & 0.000 & 0.026
\end{tabular}

Ingredients

Fish meal

$$
0.94^{\mathrm{b}}
$$

0.98

$1.07^{\mathrm{b}}$

$0.83^{\mathrm{a}}$

0.27

Soybean meal

$0.79^{\mathrm{a}}$

0.95

0.045

0.44

0.063

0.048

SEM

0.037

ab Means within diets and ingredients in the same column with different superscripts are significantly different $(P<0.05)$. SEM=Standard error of the mean. 


\section{4 .2 cpn60 clone libraries}

Three cpn60 universal target clone libraries were constructed from distal gut contents from each feed group, resulting in 1176, 1000 and 1181 high quality, complete universal target sequences from the CN, FM and SBM libraries respectively. When all the assembled sequences from the three libraries were combined, an overall total of 32 different sequences were observed, occurring at frequencies ranging from 0-1148 in the three libraries. Unique sequences identified along with their nearest neighbour in the cpnDB reference database and their observed frequencies in the three libraries are show in Table 3.4. The most frequently observed sequences overall were 97-99\% identical to Carnobacterium maltaromaticum ATCC $27865^{\mathrm{T}}$ (previously known as C. piscicola (Mora et al., 2003)) and accounted for $88 \%, 55 \%$ and $97 \%$ of the clones in the CN, FM and SBM libraries respectively. C. maltaromaticum-like sequences were the only sequences common to all three libraries. Sequences with similarity to the gamma class of Proteobacteria were also relatively abundant, comprising $10 \%, 41 \%$ and $3 \%$ of the $\mathrm{CN}, \mathrm{FM}$ and $\mathrm{SBM}$ libraries. Sequences within this group were most similar to enterobacterial genera such as Pseudomonas, Citrobacter, Hafnia, and Serratia. Aeromonas-like sequences were also detected in the $\mathrm{CN}$ and FM libraries. The CN library contained 17 different sequences, compared to 14 in FM and only 4 in SB. The FM library contained the most balanced ratio of Gamma Proteobacteria and Firmicutes (41\% and $59 \%$ of clones) while the other two libraries were more skewed toward the Firmicutes ( $88 \%$ and $97 \%$ of clones in the $\mathrm{CN}$ and SBM libraries respectively).

\section{3}


The phylogenetic relationships of rainbow trout intestinal microbiota cpn60 sequences are illustrated in Figure 3.1. 


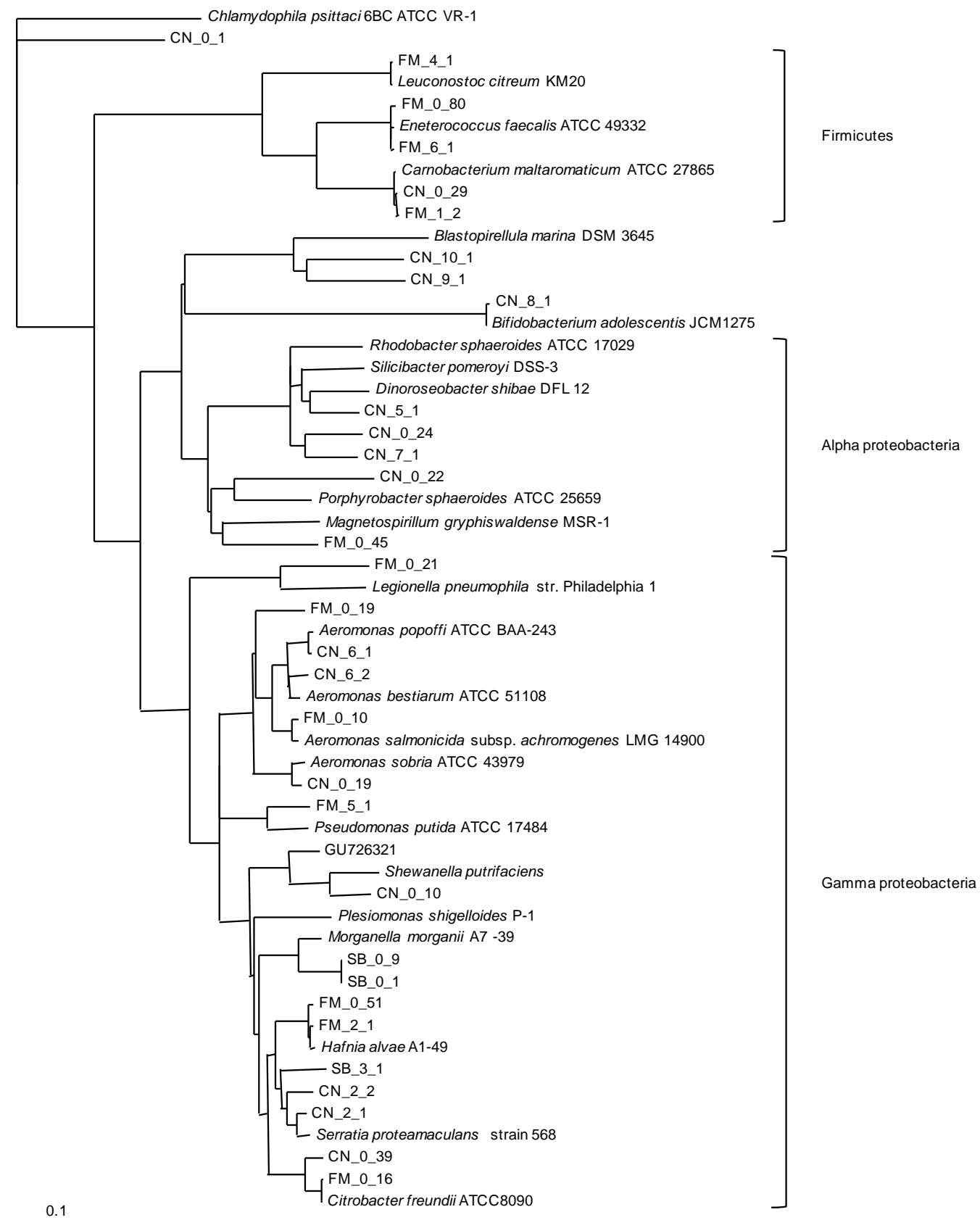

Figure 3.1 Phylogenetic tree based on the 32 unique sequences detected in the $\mathrm{CN}, \mathrm{FM}$ and $\mathrm{SB}$ libraries and related reference strain sequences. Accession numbers for the reference strains are as follows: Chlamydophila psittaci 6BC ATCC VR-1 (AY581777), Leuconostoc citreum KM20 (DQ489736), Eneterococcus faecalis ATCC 49332 (DQ074968), Carnobacterium maltaromaticum ATCC 27865 (GU911354), Blastopirellula marina (NZ_AANZ01000045), Bifidobacterium adolescentis JCM1275 (AF210319), Rhodobacter sphaeroides ATCC 17029 (NC_009049), Silicibacter pomeroyi DSS-3 (NC_003911), Dinoroseobacter shibae DFL 12 (CP000830), 
Porphyrobacter sphaeroides ATCC 25659 (EU790571), Magnetospirillum gryphiswald MSR-1 (BX640510), Legionella pneumophila str. Philadelphia 1 (NC_002942), Aeromonas popoffi ATCC BAA-243 (EU306814), Aeromonas bestiarum ATCC 51108 (EU306796), Aeromonas salmonicida subsp. achromogenes (EU306829), Aeromonas sobria ATCC 43979 (EU306834), Pseudomonas putida ATCC 17484 (EF685203), Shewanella putrifaciens (AE014299), Plesiomonas shigelloides P-1 (AF230960), Morganella morganii A7 -39 (AY245876), Hafnia alvae A1-49 (GU911356), Serratia proteamaculans strain 568 (NC_009832), Citrobacter freundii ATCC8090 (AY123708). 
Table 3.4 Unique cpn60 sequences detected in CN, FM and SB libraries.

\begin{tabular}{|c|c|c|c|c|c|c|c|}
\hline \multirow[b]{2}{*}{ Seq ID } & \multirow[b]{2}{*}{ Family } & \multirow[b]{2}{*}{ Nearest neighbor } & \multirow[b]{2}{*}{$\begin{array}{l}\% \\
\text { Identity }\end{array}$} & \multicolumn{4}{|c|}{ Number(percent) of clones } \\
\hline & & & & $\mathrm{CN}$ & FM & SBM & Total \\
\hline $\mathrm{cn}{ }_{-} 8_{-} 1$ & Actinobacteria & $\begin{array}{l}\text { Bifidobacterium } \\
\text { adolescentis }\end{array}$ & 99.5 & $1(0.1)$ & $0(0)$ & $0(0)$ & $1(<0.1)$ \\
\hline $\mathrm{cn} \_5 \_1$ & $\begin{array}{l}\text { Alpha } \\
\text { Proteobacteria }\end{array}$ & Dinoroseobacter shibae & 87.7 & $5(0.4)$ & $0(0)$ & $0(0)$ & $6(0.18)$ \\
\hline fm_0_45 & & $\begin{array}{l}\text { Magnetospirillum } \\
\text { gryphiswald }\end{array}$ & 79.0 & $1(0.9)$ & $0(0)$ & $0(0)$ & $1(<0.1)$ \\
\hline $\mathrm{cn}{ }_{-}{ }_{-} 22$ & & $\begin{array}{l}\text { Porphyrobacter } \\
\text { sanguineus }\end{array}$ & 77.8 & $10(0.9)$ & $0(0)$ & $0(0)$ & $1(<0.1)$ \\
\hline $\mathrm{cn}{ }_{-}{ }_{-}{ }_{2} 4$ & & Rhodobacter sphaeroides & 85.7 & $4(0.3)$ & $0(0)$ & $0(0)$ & $10(0.3)$ \\
\hline $\mathrm{cn}{ }_{-} 7_{-} 1$ & & Silicibacter pomeroyi & 84.0 & $1(0.1)$ & $0(0)$ & $0(0)$ & $4(0.12)$ \\
\hline $\mathrm{fm}_{-}{ }_{-}{ }_{-}$ & Firmicutes & $\begin{array}{l}\text { Carnobacterium } \\
\text { maltaromaticum }\end{array}$ & 99.0 & $0(0)$ & $13(1.3)$ & $0(0)$ & $13(0.4)$ \\
\hline cn_0_29 & & $\begin{array}{l}\text { Carnobacterium } \\
\text { maltaromaticum }\end{array}$ & 97.3 & $1032(87.8)$ & $537(53.7)$ & $1148(97.2)$ & $2717(80.9)$ \\
\hline $\mathrm{fm} \mathrm{m}_{-}{ }_{-} 1$ & & Enterococcus faecalis & 99.6 & $0(0)$ & $17(1.7)$ & $0(0)$ & $17(0.5)$ \\
\hline $\mathrm{fm} \mathrm{m}_{-}{ }_{-} 80$ & & Enterococcus faecalis & 99.3 & $0(0)$ & $1(0.1)$ & $0(0)$ & $1(<0.1)$ \\
\hline $\mathrm{fm}_{-}{ }_{-} \_1$ & & Leuconostoc citreum & 99.8 & $0(0)$ & $23(2.3)$ & $0(0)$ & $23(0.7)$ \\
\hline $\mathrm{cn} \_6 \_2$ & $\begin{array}{l}\text { Gamma } \\
\text { Proteobacteria }\end{array}$ & Aeromonas bestiarum & 96.2 & $2(0.2)$ & $0(0)$ & $0(0)$ & $2(0.1)$ \\
\hline $\mathrm{cn}{ }_{-} 6_{-} 1$ & & Aeromonas popoffi & 99.0 & $2(0.2)$ & $0(0)$ & $0(0)$ & $2(0.1)$ \\
\hline $\mathrm{fm} \mathrm{m}_{-}{ }_{-} 10$ & & Aeromonas salmonicida & 98.4 & $0(0)$ & $12(1.2)$ & $0(0)$ & $12(0.4)$ \\
\hline fm_0_19 & & Aeromonas salmonicida & 90.0 & $0(0)$ & $1(0.1)$ & $0(0)$ & $1(<0.1)$ \\
\hline $\mathrm{cn} \_0 \_19$ & & Aeromonas sobria & 96.9 & $17(1.5)$ & $0(0)$ & $0(0)$ & $17(0.5)$ \\
\hline $\mathrm{fm} \mathrm{m}_{-}{ }_{-} 16$ & & Citrobacter freundii & 99.8 & $0(0)$ & $4(0.4)$ & $0(0)$ & $4(0.1)$ \\
\hline $\mathrm{fm}{ }_{-}{ }_{-}{ }_{-1}$ & & Hafnia alvae & 98.9 & $0(0)$ & $365(36.5)$ & $0(0)$ & $365(10.9)$ \\
\hline $\mathrm{fm} \mathrm{m}_{-}{ }_{-} 51$ & & Hafnia alvae & 98.4 & $0(0)$ & $1(0.1)$ & $0(0)$ & $1(<0.1)$ \\
\hline $\mathrm{fm} \_0 \_21$ & & Legionella pneumophila & 81.0 & $0(0)$ & $1(0.1)$ & $0(0)$ & $1(<0.1)$ \\
\hline sb_0_9 & & Morganella morganii & 91.7 & $0(0)$ & $0(0)$ & $1(0.1)$ & $1(<0.1)$ \\
\hline sb_0_1 & & Morganella morganii & 91.7 & $0(0)$ & $0(0)$ & $27(2.3)$ & $27(0.8)$ \\
\hline $\mathrm{cn}{ }_{-}{ }_{-}{ }_{-} 2$ & & Plesiomonas shigelloides & 99.8 & $1(0.1)$ & $0(0)$ & $0(0)$ & $1(<0.1)$ \\
\hline $\mathrm{fm}_{-} 5_{-} 1$ & & Pseudomonas putida & 93.0 & $0(0)$ & $21(2.1)$ & $0(0)$ & $21(0.6)$ \\
\hline $\mathrm{cn} \_2 \_1$ & & Serratia proteamaculans & 96.9 & $61(5.2)$ & $3(0.3)$ & $0(0)$ & $64(1.9)$ \\
\hline $\mathrm{cn} \_2 \_2$ & & Serratia proteamaculans & 93.9 & $3(0.3)$ & $0(0)$ & $0(0)$ & $3(0.1)$ \\
\hline sb_3_ 1 & & Serratia proteamaculans & 91.2 & $0(0)$ & $0(0)$ & $5(0.4)$ & $5(0.2)$ \\
\hline $\mathrm{cn} \_0 \_39$ & & Serratia proteamaculans & 88.5 & $1(0.1)$ & $0(0)$ & $0(0)$ & $1(<0.1)$ \\
\hline $\mathrm{cn}{ }_{-} 0_{-} 10$ & & Shewanella putrifaciens & 89.2 & $31(2.6)$ & $0(0)$ & $0(0)$ & $31(0.9)$ \\
\hline $\mathrm{cn} \_10_{-} 1$ & Other & Blastopirellula marina & 77.3 & $2(0.2)$ & $0(0)$ & $0(0)$ & $2(0.1)$ \\
\hline $\mathrm{cn}{ }_{-}{ }_{-}{ }_{1}$ & & Blastopirellula marina & 76.0 & $2(0.2)$ & $0(0)$ & $0(0)$ & $2(0.1)$ \\
\hline $\mathrm{cn}{ }_{-} 0_{-} 1$ & & Chlamydophila psittaci & 69.0 & $0(0)$ & $1(0.1)$ & $0(0)$ & $1(<0.1)$ \\
\hline
\end{tabular}




\subsubsection{Quantitative PCR of intestinal microbiota}

Four targets were selected from the three libraries for quantification by qPCR in individual fish represented in the sample pools used to construct the libraries (Table 3.2). Targets were chosen to represent very abundant as well as medium abundance and rare sequences in the libraries. Based on ten-fold dilution series of cloned target sequences, the detection limit of the assays was

determined to be $5 \times 10^{2}$ target copies $\mathrm{g}^{-1}$ of intestinal contents. All four assays were applied to the 13 distal gut contents DNA extracts from individual fish that comprised the library pools (4 from CN, 6 from FM and 3 from the SBM group). Insufficient DNA extract was available to conduct the assays on the remaining 14 individual fish (Figure 3.2). 


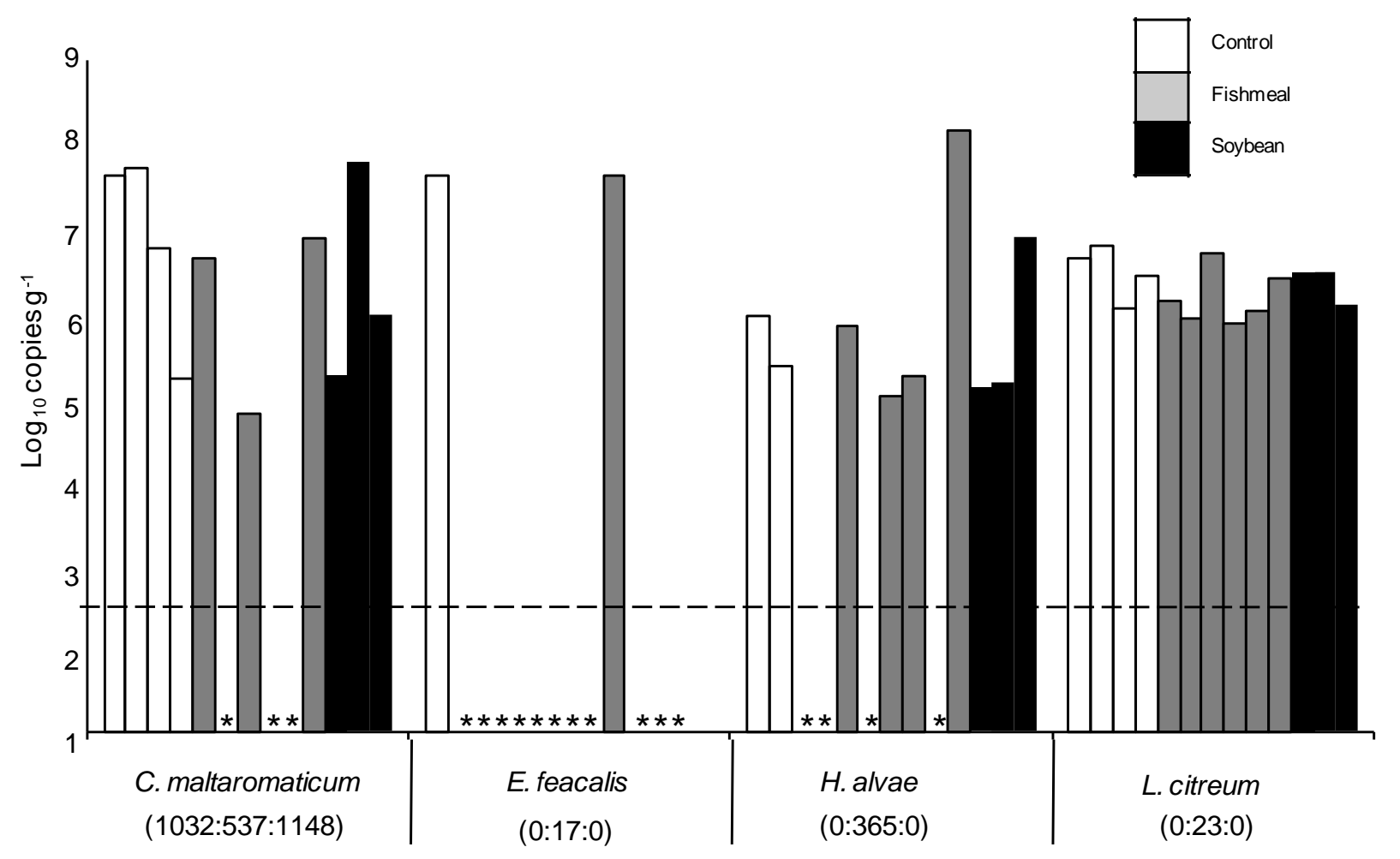

Figure 3.2 Quantitative PCR detection of four bacterial targets in intestinal contents of individual fish comprising the cpn 60 library pools. The frequency of each target sequence in the three libraries (CN : FM : SB) is indicated in parentheses below the target name. Quantities reported are the average of two replicates. The broken line indicates the detection limit of the assay. $*=$ not detected. 
C. maltaromaticum, which was seen as the most abundant bacterial sequence in the clone libraries, was the most abundant (of the four species detected) in some fish. Quantities of $C$. maltaromaticum detected ranged from undetectable to $6.26 \times 10^{6} \mathrm{~g}^{-1}$. All targets were detected in at least one individual in each diet group, except for E. faecalis, which was not detected in any of the SBM diet group fish. Enormous variation in target abundance was observed between individual fish for three of the targets. In contrast, detected quantities of Leuconostoc citreum-like sequences were consistent, detected in all individual samples and ranging from $7.53 \times 10^{4} \mathrm{~g}^{-1}$ to $6.04 \times 10^{5} \mathrm{~g}^{-1}$.

\subsubsection{Host gene expression}

Expression of PCNA, IL-1 $\beta$, and IgM was examined in mid gut and distal gut tissues of all individual fish. Quantification was expressed relative to housekeeping gene $\beta$-actin and the mean copy number \pm SEM is reported in Table 3.5. Variation in gene expression levels between individual fish was high for all gene targets. Expression of PCNA was significantly increased $(P<0.05)$ in the mid gut of animals fed the reference diet relative to the fish meal and soybean meal diets. A non-significant trend $(0.10<P>0.05)$ was also observed for IL-1 $\beta$ in the same group. No other significant differences or trends in gene expression were observed. 
Table 3.5 Expression of target genes in intestine of rainbow trout fed control (CN), fish meal (FM), and soybean meal (SBM) diets ( $n=9$ for each diet).

\begin{tabular}{|c|c|c|c|c|c|c|}
\hline \multirow{2}{*}{$\begin{array}{l}\text { Gene } \\
\text { target }\end{array}$} & \multicolumn{3}{|c|}{$\overline{\text { Midgut }}$} & \multicolumn{3}{|c|}{ Distal gut } \\
\hline & $\mathrm{CN}$ & FM & SBM & $\mathrm{CN}$ & FM & SBM \\
\hline $\operatorname{IgM}$ & $977 \pm 237$ & $941 \pm 333$ & $717 \pm 165$ & $2060 \pm 675$ & $1181 \pm 367$ & $2062 \pm 708$ \\
\hline $\mathrm{IL}-1 \beta$ & $2010 \pm 1057^{*}$ & $135 \pm 25^{*}$ & $225 \pm 45^{*}$ & $544 \pm 201$ & $463 \pm 121$ & $292 \pm 74$ \\
\hline PCNA & $1101 \pm 194^{\mathrm{a}}$ & $537 \pm 116^{\mathrm{b}}$ & $440 \pm 73^{b}$ & $1396 \pm 393$ & $548 \pm 77$ & $1082 \pm 243$ \\
\hline
\end{tabular}

Values are mean $\beta$-act normalized copy numbers \pm SEM

ab Means in the same row with different superscripts are significantly different $(P<0.05)$

(Tukey's HSD test)

* Means in the same row with an asterisk show a non-significant trend $(0.10<P>0.05)$ 
3.4.5 Correlations between diets, host gene expression and intestinal microbiota

Correlations between all pairs of experimental parameters were calculated and significant correlations are reported in Table 3.6 and Figure 3.3. All correlations between IL- $1 \beta$ in mid and distal gut and IgM in mid and distal gut showed significant positive correlations $(P<0.05)$. The semi-purified reference diet was positively correlated with PCNA expression in the mid gut and negatively correlated with $H$. alvae numbers in the distal gut. In contrast, the fish meal diet was positively correlated with the numbers of $H$. alvae and negatively correlated with the numbers of $C$. maltaromaticum. The numbers of $H$. alvae and $L$. citreum were positively correlated with each other and $H$. alvae was negatively correlated with IL-1 $\beta$ in the mid gut while L. citreum was negatively correlated with IL- $1 \beta$ in the distal gut. Finally, PCNA in the distal gut was significantly positively correlated with $\operatorname{IgM}$ in the distal gut. 
Table 3.6 Two-tailed correlations between diets, intestinal gene expression and intestinal bacterial populations. Only significant correlations $(P<0.05)$ are shown.

\begin{tabular}{|c|c|c|c|c|c|c|c|c|}
\hline Parameters & & Reference & Fish meal & $\begin{array}{l}\text { IgM } \\
\text { Mid gut }\end{array}$ & $\begin{array}{l}\text { IgM } \\
\text { Distal gut }\end{array}$ & $\begin{array}{l}\text { IL- } 1 \beta \\
\text { Mid gut }\end{array}$ & $\begin{array}{l}\text { IL } 1 \beta \\
\text { Distal gut }\end{array}$ & H. alvae \\
\hline $\begin{array}{l}\mathrm{r} \\
P \text {-value }\end{array}$ & IgM Distal gut & & & $\begin{array}{l}0.581 \\
0.003\end{array}$ & & & & \\
\hline $\begin{array}{l}\mathrm{r} \\
P \text {-value }\end{array}$ & IL- $1 \beta$ Mid gut & & & $\begin{array}{r}0.868 \\
<0.001\end{array}$ & $\begin{array}{r}0.648 \\
<0.001\end{array}$ & & & \\
\hline $\begin{array}{l}\mathrm{r} \\
P \text {-value }\end{array}$ & IL- $1 \beta$ Distal gut & & & $\begin{array}{r}0.885 \\
<0.001\end{array}$ & $\begin{array}{r}0.651 \\
<0.001\end{array}$ & $\begin{array}{r}0.992 \\
<0.001\end{array}$ & & \\
\hline $\begin{array}{l}\mathrm{r} \\
P \text {-value }\end{array}$ & PCNA Mid gut & $\begin{array}{l}0.474 \\
0.017\end{array}$ & & & & & & \\
\hline $\begin{array}{l}\mathrm{r} \\
P \text {-value }\end{array}$ & PCNA Distal gut & & & & $\begin{array}{l}0.482 \\
0.02\end{array}$ & & & \\
\hline $\begin{array}{l}\mathrm{r} \\
P \text {-value }\end{array}$ & C. maltaromaticum & & $\begin{array}{l}-0.717 \\
0.045\end{array}$ & & & & & \\
\hline $\begin{array}{l}\mathrm{r} \\
P \text {-value }\end{array}$ & H. alvae & $\begin{array}{l}-0.68 \\
0.002\end{array}$ & $\begin{array}{l}0.553 \\
0.017\end{array}$ & & & $\begin{array}{r}-0.477 \\
0.046\end{array}$ & & \\
\hline $\begin{array}{l}\mathrm{r} \\
P \text {-value }\end{array}$ & L. citreum & & & & & & $\begin{array}{r}-0.638 \\
0.026\end{array}$ & $\begin{array}{l}0.723 \\
0.012\end{array}$ \\
\hline
\end{tabular}




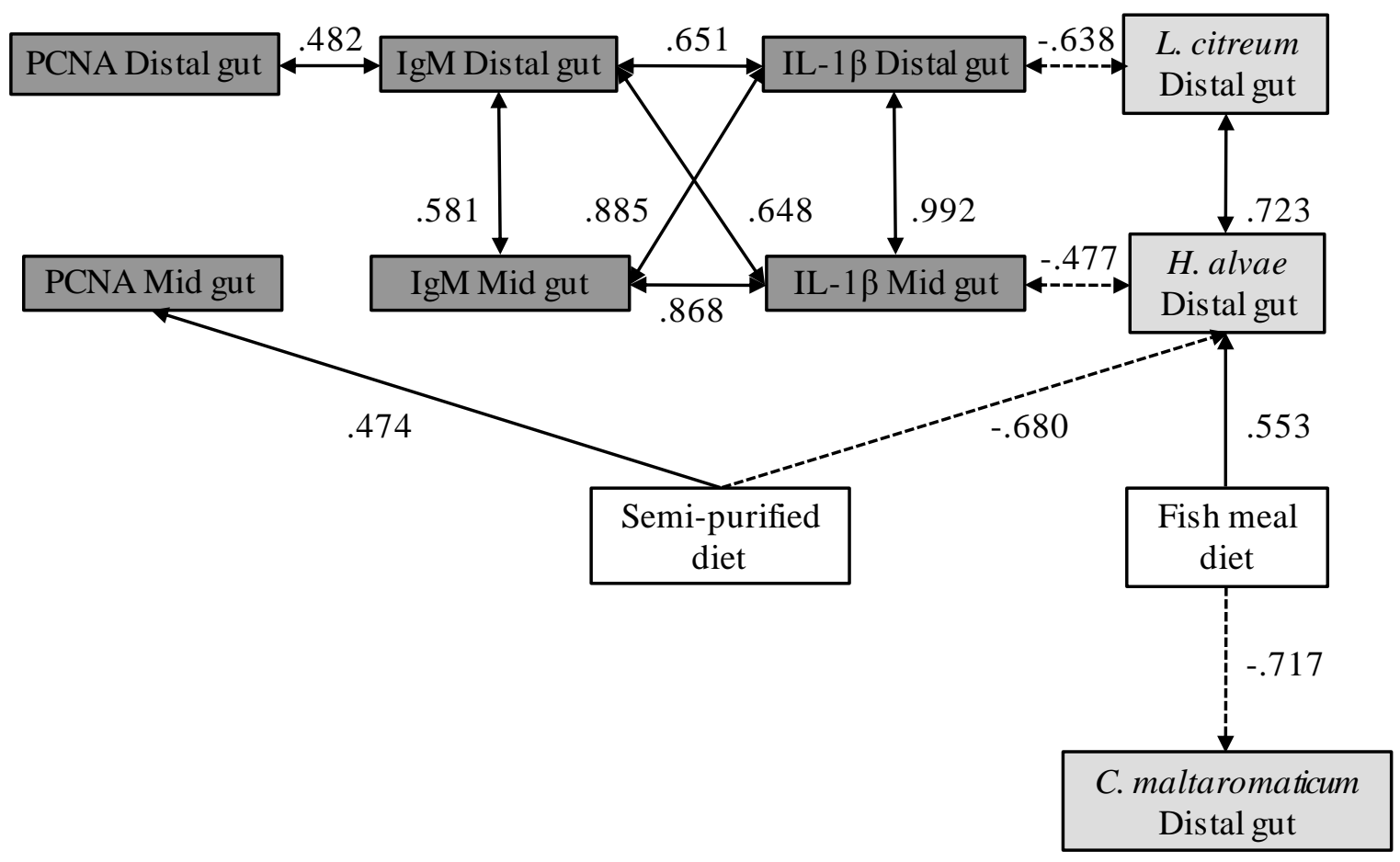

Figure 3.3 The relationships between significant correlations $(P<0.05)$ of diets, intestinal gene expression and intestinal bacterial populations in rainbow trout. The numbers are correlation coefficients (r). Solid lines indicate positive correlations while broken lines indicate negative correlations. 


\subsection{Discussion}

Because of the wide range of microbiota descriptions in rainbow trout studies and the established effects of management style, diet and other variables on the microbiota, it is essential to collect detailed information on the host and intestinal microbiota characteristics of any particular experimental system prior to drawing conclusions from experiments in which these factors are manipulated. During the digestibility trial described in this manuscript, we evaluated the digestibility of soybean meal and fish meal and also characterized the intestinal contents microbiota and fish inflammatory marker gene expression for 27 individual fish in three diet groups.

In the current study, a semi-purified reference diet was used. Such diets have been shown to be associated with poor feeding activity and growth performance in some fish so feed attractants have been required (Kaushik. 1998). The NRC (1993) suggests that the use of fish meal to remedy palatability issues with purified diets. However, the use of a reference diet containing soybean meal and/or fish meal would have confounded our measurements of intestinal microbiota and gene expression so a purified diet was used to eliminate this concern. The purified reference diet chosen is similar to those employed in other studies, primarily those focused on determining vitamin and amino acid requirements of finfish (Kim, et al. 1991; Kim, et al. $1992 \mathrm{a}$; Kim, et al. $1992 \mathrm{~b}$; Kaushik. 1998).

Results of these studies have led these researchers to wonder whether purified reference diet unpalatability is related to sensory attributes of the diets 
or a negative feedback response due to gastrointestinal upset. Krogdahl et al. (Krogdahl, et al. 2003) found when soybean meal is included at levels as low as $10 \%$ in the diet of Atlantic salmon (Salmo salar), factors such as feed intake, growth, digestibility and gut histology and enzyme activity were negatively impacted. As the digestibility values between the purified reference diet and the soybean meal test diet did not vary significantly, and evidence shows fish fed a purified reference diet have significant differences in growth from fish fed diets containing fish meal but not a significantly different feed to gain ratio (Kim, et al. 1991), further histological investigation may be warranted.

Purified diets are formulated to meet the known nutritional requirements of salmonids, yet our results suggest that nutritional information in addition to proximate, amino acid, vitamin and mineral composition is required. For example, Aksnes et al. (Aksnes, et al. 2006) described low molecular weight pronutritional factors present in fish meal that have an impact on growth performance of salmonids. They might also affect intestinal microbial ecology and gene expression. Furthermore, antinutritional factors present in soybean meal and other plant protein sources contain antinutritional factors (Drew, et al. 2007) which may negatively affect growth performance and intestinal microbiota. Despite the proposed nutrient suitability of the reference diet, our results show it was less available nutritionally than the fish meal test diet. These results should be taken into consideration with choosing reference diets for specific types of feeding trials in rainbow trout. As the digestibility calculation used in this trial (Forster. 1999) is a relative measurement whereby 
the nutrient utilization of specific ingredients are compared with one another based on their relationship to the reference diet, our findings suggest the semipurified reference diet may be useful in digestibility studies examining the use of novel feed ingredients but is not nutritionally equal to a diet containing fish meal.

Estimates of the culturable proportion of the intestinal microbiota of trout are 50\% or less (Spanggaard, et al. 2000; Navarrete, et al. 2009; Navarrete, et al. 2010), making culture-independent molecular methods essential for gaining a comprehensive understanding of this ecology. Application of a wide range of methods in a number of studies have led to somewhat of a consensus on the rainbow trout intestinal microbiota: that it is primarily composed of Gamma Proteobacteria and Firmicutes, particularly lactic acid bacteria (Spanggaard, et al. 2000; Huber, et al. 2004; Heikkinen, et al. 2006; Pond, et al. 2006; Kim, et al. 2007; Navarrete, et al. 2010). Results of the current study are consistent with these observations. Although some Actinobacteria and Alpha Proteobacteria sequences were detected in the control library, the majority of cpn60 sequences in the clone libraries corresponded to Firmicutes and Gamma Proteobacteria. The Gamma Proteobacteria sequences were more diverse (18 different sequences) compared to the Firmicutes (5 different sequences) which were dominated by sequences nearly identical to Carnobacterium maltaromaticum (formerly C. piscicola (Table 3.4). Greater diversity of sequences was detected in the $\mathrm{CN}$ and FM libraries (16 and 14 sequences) than in the SB library in which only 4 different sequences were detected. The FM 
library contained nearly equal proportions of Firmicutes and Gamma Proteobacteria sequences, suggesting that this diet supported a more phylogenetically diverse microbiota and that the reference diet and soybean meal diets were only to able to support a narrower range of bacterial diversity. Relatively small numbers of clones were also examined from libraries generated from mid gut contents (approximately $100-200$ clones each). The taxa identified and relative abundances of these groups were very similar to the distal gut libraries described suggesting that the mid gut and distal gut microbiota are similar in structure in these fish (data not shown).

Improvements in culture-based methods have led to increased recognition of lactic acid bacteria such as Lactobacillus, Lactococcus, Leuconostoc, and Enterococcus in fish intestinal microbiota (Ringo, et al. 1998). Among the other lactic acid bacteria, Carnobacterium are common commensals of fish including trout (Ringo, et al. 2001) where they have been detected in intestinal contents (Spanggaard, et al. 2000; Kim, et al. 2007) and in the mucosal microbial community (Pond, et al. 2006). They are also considered for their potential as probiotics for salmonids (Robertson, et al. 2000). Speciation within this genus is not often performed since it can be difficult using phenotypic methods or $16 \mathrm{~S}$ rRNA sequence. 16S rRNA sequences of the two most common fish commensals, $C$. divergens and $C$. maltaromaticum, are $\sim 98 \%$ identical. $C$. maltaromaticum is easily distinguished from $C$. divergens by cpn60 sequence (only $84 \%$ identical). This degree of sequence difference is easily exploited in 
the development of species-specific qPCR assays such as those employed in this study.

Interestingly, despite its abundance in the clone libraries, $C$. maltaromaticum was not detected in all of the individual fish that comprised the template pools used in library construction (Figure 3.2). It was not detected in three of the six FM individuals examined. This could be because the organism is not present in these individuals, but a more likely explanation is that it was present below the detection limit of the assay $\left(5 \times 10^{2}\right.$ copies $\left.\mathrm{g}^{-1}\right)$. Another possibility is that the assay was too specific, detecting only some strains of $C$. maltaromaticum. Intraspecific sequence variation that could be responsible for our observation has been reported in other bacterial species identified in natural microbial communities (Vermette, et al. 2010). However, little cpn60 sequence variation was detected in the C. maltaromaticum-like sequences, with only 2 different sequences detected. This is consistent with our examination of cultured Carnobacterium isolates from fish in the same facility which indicated a homogenous population based on cpn60 sequence (data not shown).

Overall, a large amount of variation was observed in the quantities of the four targets in the 13 individual fish examined, with the exception of L. citreum, which was consistent among all individuals. Variation in microbiota between rainbow trout on the same diet has been reported previously and found to be a complicating factor in evaluating the effects of feed ingredients on the microbiota and intestinal health (Heikkinen, et al. 2006). In our study we focused on the microbial community associated with intestinal contents rather 
than the attached mucosal communities and luminal communities may be more variable over time and between individuals due to the more dynamic nature of the luminal environment. The inconsistent relationship between qPCR results and cloned sequence frequency (Figure 3.2) illustrates the "pooling effect" frequently observed in studies where pooled samples from many individuals are used to generate clone libraries. In these cases, the inclusion of one individual with an abundance of a particular species can result in an over-representation of the corresponding sequence in the library (Desai, et al. 2009). This is certainly true of E. faecalis, which was present at detectable levels in only one of the FM individuals but accounted for almost $2 \%$ of the cloned sequences in the FM library. Also, all PCR-based methods are subject to biases in amplification efficiencies of various template DNAs, although in this case, the cpn60 library was made using techniques optimized to reduce some of this bias (Hill, et al. 2006). Although the generation of clone libraries from pooled samples is not a quantitative approach, it does serve to give a general overview of the relative abundances of population constituents and more importantly, provides reference data for other quantitative approaches such as qPCR analysis.

Another aspect of this study was to examine the expression of host genes associated with epithelial damage and inflammation in intestinal tissue. Negative effects on gut epithelium morphology, increases in PCNA and IgM in the distal gut of Atlantic salmon on soybean-based diets have been reported (Bakke-McKellep, et al. 2007). Similar histological changes but with no performance effect have been reported in rainbow trout (Heikkinen, et al. 2006). 
These previous studies of inflammatory indicators have been based on protein detection (by ELISA (IgM) or immunohistochemistry (PCNA)). In the current study, we employed qPCR to measure transcript levels relative to the housekeeping gene $\beta$-actin. As illustrated in Table 3.5, enormous variation in gene expression levels was detected, making the recognition of trends in the data difficult. Interestingly, differences were only observed in the mid gut, although the distal gut has generally been recognized as the site of greate st inflammatory impact (Heikkinen, et al. 2006; Bakke-McKellep, et al. 2007). PCNA expression was significantly higher in mid gut tissue from reference diet fish than in fish consuming the fish meal or soybean meal diets $(P<0.05)$ and a similar but non-significant trend $(0.10<P>0.05)$ was observed for IL- $1 \beta$. This is suggestive of inflammation in the mid gut of fish fed the reference diet. The lack of an obvious soybean meal effect may be because it was obscured by the effects of the synthetic basal diet, which was chosen for its value in design of a digestibility trial, not a trial optimized for investigating inflammation. No significant differences in gene expression in the distal gut tissues were associated with diet, perhaps not surprisingly given the large variation detected within diet groups.

The results ind icate that diet composition may alter host gene expression and intestinal microbiota. However, the relationships between these different parameters are difficult to perceive thus, correlation analysis was used to determine relationships between parameters with the caveat that these correlations do not indicate cause and effect but merely associations. The 
inflammatory marker genes IgM, IL-1 $\beta$ were highly correlated with each other in all two-way comparisons. This relationship has not been previously reported and bears further examination. There were no direct correlations between diets and inflammatory gene expression. Rather, the effect of diet appeared to be mediated through the effect of diet on the microbiota, represented by $H$. alvae which in turn was correlated with L. citreum. Intestinal microbiota can modulate the expression of IL-1 $\beta$ in zebrafish (Brugman, et al. 2009). It is therefore reasonable to assume that inflammation of the gut reported in salmonids fed vegetable-based diets (Baeverfjord, et al. 1996; Bakke-McKellep, et al. 2000; Bakke-McKellep, et al. 2007) might be due to both the direct effect of diet and the indirect effect of alteration of the gut microbiota by diet.

Intestinal bacteria use the undigested portion of the diet as energy substrates. Thus the "indigestibility coefficient" or (1 - ADC) describes the portion of each nutrient that is available to the intestinal microbiota. The indigestibility coefficients for the purified, fish meal and soybean meal diets respectively are $0.23,0.18$ and 0.23 for dry matter and $0.08,0.05$ and 0.07 for crude protein. The chemical composition of the undigested portion of the feed changes as well. The soybean meal diet and the purified diet have equal indigestibility coefficients of 0.23 . However, for the soybean meal diet, $30 \%$ of this fraction is derived from soybean meal which contains approximately $29 \%$ total non-starch polysaccharides, consisting of $15 \%$ pentosans, $20 \%$ cellulose and $21 \%$ pectin (Malathi, et al. 2001). These substrates may support the 
proliferation of different bacterial species in the intestinal tract. This is supported by the results of the current study.

A key finding that resulted from the application of qPCR, for both inflammatory markers and in the microbiota study, is that we were able to describe quantitatively the fish-to-fish variation in our experiment. This emphasizes that it may be critical to examine individual fish to have sufficient analytical power to determine relationships between diet, host and microbiota. An individual approach will permit the recognition of species within the microbiota that are significantly affected by diet changes or are associated with health and performance. 


\section{Chapter 4 Effects of plant-based diets on the distal gut microbiome of rainbow trout (Oncorhynchus mykiss).}

Chapter 4 is a part of a multi-disciplinary study, and has been submitted for publication.

Desai, A., Links, M.G., Collins, S.A., Mansfield, G., Drew, M., Van Kessel, A.G. and Hill, J.E. 2011. Effects of plant-based diets on the distal gut microbiome of rainbow trout (Oncorhynchus mykiss). Submitted.

Author contributions

All authors participated in the design of the experiments and contributed to writing of the manuscript. Collins was responsible for diet formulations described in Table 4.1. Initial processing and assembling of the raw data was done by Links.

The author of this thesis contributed to in diet making, fish husbandry and sample collection. All other data presented in this manuscript are the work of the thesis author. 


\subsection{Abstract:}

Replacement of fish meal in aquaculture diets with plant proteins is economically desirable, but the effects of alternative protein sources on the intestinal microbiota and fish health are poorly understood. We examined the intestinal microbiome of 108 rainbow trout (Oncorhynchus mykiss) fed with diets including plant ingredients (peas, soybean, canola) at two processing levels (meal, concentrate), or a fish meal (FM) control diet. Microbial community profiles were determined using pyrosequencing of cpn60 PCR products and $16 \mathrm{~S}$ rRNA DGGE. Microbial profiles of fish fed the FM diet changed over the course of the study with reduction in species richness and diversity and therefore evaluation of the effects of experimental diets was accomplished through comparison of each diet with its concurrent FM control. Plant ingredient diets were associated with higher Firmicutes:Proteobacteria ratios than controls. Both DGGE and pyrosequencing data showed that microbiomes of fish fed meal-based diets were more distinguishable from the FM control microbiomes than were the microbiomes of fish fed protein concentrate diets. Changes in the FM diet associated profiles could be the result of age-related physiological changes in these fish or a long-term effect of the change in environment from outdoor aquaculture facility to an indoor $\mathrm{re}$ circulating facility. Regardless of the reason, these observations have significant implications for future evaluations of diets and ingredients. We also demonstrated changes in the intestinal microbiome that may contribute to negative health outcomes when diets contain plant meal proteins. These changes 
in microbiome structure can be minimized with additional processing of plant ingredients. 


\subsection{Introduction}

The Food and Agriculture Organization of the United Nations has declared the important role of the aquaculture sector in achieving global food availability, economic growth, and improving living standards of people in developed as well as developing countries around the globe (FAO. 2000). This rapidly developing agricultural sector directly depends on the aquafeed industry, which in turn depends on fish meal (FM) as its primary protein source. Since the global supply of FM is insufficient to meet the demands of a growing industry, there is significant interest in finding alternative protein sources appropriate for carnivorous aquaculture fish (Tacon, et al. 2008). A major obstacle to this work is that these plant-based diets induce a variety of histological and functional changes in the gastrointestinal tracts of fish. Observed effects include change in the intestinal structure, inflammation, reduced growth as well as nutrient digestion and absorption, and an increased susceptibility to disease (Krogdahl, et al. 2000; Nordrum, et al. 2000). These changes are thought to be due to direct effects of anti-nutritional factors in plant ingredients and/or the indirect result of diet-induced changes in the structure and function of the intestinal microbiota (Olsen, et al. 2001; Ringo, et al. 2006c). Plant ingredients such as soybean, canola and peas have been used previously in manufacturing of aquaculture diets (Thiessen, et al. 2003; Mundheim, et al. 2004; Gatlin, et al. 2007). These ingredients are known to contain anti-nutritional factors such as saponins, glucosinolates, phytate and trypsin inhibitors, although processing the ingredients can reduce their levels. Previous studies have shown that soy protein 
concentrate (SPC) and canola protein concentrate (CPC) can be used at maximum inclusion rates of $50 \%$ and pea protein concentrate (PPC) at $27 \%$ without having a negative impact on fish growth (Mambrini, et al. 1999; Thiessen, et al. 2003; Krogdahl, et al. 2010). However, the effects of the se ingredients on intestinal physiology and microbiota of salmonid fish have not been investigated.

There is a long history of the application of culture-based techniques to understand the structure and dynamics of the intestinal microbiota of fish (Cahill. 1990); however, these methods give a limited picture of the intestinal microbiota since only culturable bacterial species are detected. Molecular techniques such as clone library sequencing, temperature gradient gel electrophoresis (TGGE), restriction fragment length polymorphism (RFLP), and fluorescence in situ hybridization (FISH) have also been used to characterize the dominant bacterial components of the rainbow trout intestinal microbiota (Huber, et al. 2004; Mansfield, et al. 2010; Navarrete, et al. 2010). However, there are few reports on the effect of diet on the intestinal microbiota of fish (Heikkinen, et al. 2006; Bakke-McKellep, et al. 2007; Dimitroglou, et al. 2009)

The cpn60 universal target (UT) has been exploited in characterizing microbial communities associated with environments such as the urogenital and digestive systems of humans and other terrestrial animals (Hill, et al. 2005a; Hill, et al. 2005b; Dumonceaux, et al. 2006a; Desai, et al. 2009). The cpn60 gene has been established as an informative gene target that has more discriminating power than the $16 \mathrm{~S}$ rRNA gene for closely related species (Hill, 
et al. 2004). We recently reported a cpn60 clone library based characterization of the effects of soybean meal and fish meal diets on rainbow trout distal gut microbiota (Mansfield, et al. 2010). An important observation in this study was substantial animal-to-animal variation in the relative abundances of identified bacterial sequences (detected by qPCR), suggesting that analysis of individual fish rather than pooled samples would be critical to support conclusions regarding diet effects on the intestinal microbiome. Pyrosequencing technology has now made large-scale sequencing of many samples feasible and this technique has been successfully adopted to take advantage of the discriminating power of the cpn60 UT (Schellenberg, et al. 2009; Schellenberg, et al. 2011a; Chaban, et al. 2012).

We hypothesized that the negative performance and health outcomes reported when fish are fed plant-based ingredients are mediated in part by changes in the profile of intestinal microbiota. The objective of this study was to evaluate changes in the structure of the intestinal microbiota of rainbow trout associated with different plant-based diets relative to a FM control diet. Results support the conclusion that meal forms of plant ingredients disrupt the intestinal microbiome, however, further processing to protein concentrate forms minimizes disruptions in the intestinal microbiome compared to a "gold standard" FM diet. Our results support further development of plant protein processing techniques for aquaculture diets. 


\subsection{Materials and methods}

\subsubsection{Fish Management}

Triploid female rainbow trout (Onchorhynchus mykiss) were purchased from the Wild West Steelhead fish farm (Lucky Lake, SK, Canada). The average weight of fish during the course of the experiment increased from $510 \mathrm{~g}$ to 1,100 g. All trials were conducted at the Prairie Aquaculture Research Centre (PARC, University of Saskatchewan, Saskatoon, SK), a bio-filtered, recirculating aquaculture system. Fish were housed in $360 \mathrm{~L}$ tanks with water temperature adjusted at $15 \pm 1{ }^{\circ} \mathrm{C}$ with photoperiod of $14 \mathrm{~h}$ light and 10 hours dark. All instructions and guidelines set by the Canadian Council of Animal Care (CCAC, 2005) were followed throughout the experimental trial (University Committee on Animal Care and Supply Protocol 19980142). All fish were hand-fed to satiety twice daily.

\subsubsection{Growth Experiments}

Each plant ingredient (soybean, pea or canola) was fed as either a ground meal or protein concentrate. Diets containing fish meal $(390 \mathrm{~g} / \mathrm{kg}$ Nova Scotia herring meal; Shur-Gain Aquaculture, Truro, NS, Canada or $400 \mathrm{~g} / \mathrm{kg}$ West Coast Fish Meal; Federated Co-Operative Ltd., Saskatoon, SK, Canada) were used as a control. Two sources of fish meal were necessary to provide adequate amounts for diet formulation for the entire experiment. The experimental diets were formulated to contain $300 \mathrm{~g} / \mathrm{kg}$ of their respective plant ingredients. All 
diets were formulated to contain $17.58 \mathrm{MJ} / \mathrm{kg}$ digestible energy and $386.2 \mathrm{~g} / \mathrm{kg}$ of digestible crude protein and met or exceeded the nutrient requirements for rainbow trout (NRC (National Research Council). 1993) (Table 4.1). The feeding trials were conducted over a 12 month period starting with pea meal (PM) and PPC, followed by soybean meal (SBM), SPC and canola meal (CM), and concluding with CPC (Figure 4.1). A control group fed with the FM diet was included concurrently with each experimental diet. FM diet replicates associated with each experimental diet are annotated with subscripts, e.g. FM $_{S B M}$ is the FM control diet group concurrent with the SBM trial. Each feeding trial lasted 56 days with a rest period of 4 to 6 weeks between trials. Three tanks were randomly assigned to each diet. The same fish were used throughout the experiment and were re-randomized by tank and diet before each experiment. 
Table 4.1 Ingredient composition of experimental diets.

\begin{tabular}{|c|c|c|c|c|c|c|c|c|}
\hline Ingredient $(\mathrm{g} / \mathrm{kg})$ & $\mathrm{FM}^{*}$ & $\mathrm{PM}$ & $\overline{P P C}$ & SBM & $\mathrm{FM}^{\%}$ & $\overline{\mathrm{SPC}}$ & $\overline{\mathrm{CM}}$ & $\overline{\mathrm{CPC}}$ \\
\hline Pea meal ${ }^{a}$ & 0.00 & 300.00 & 0.00 & 0.00 & 0.00 & 0.00 & 0.00 & 0.00 \\
\hline $\begin{array}{l}\text { Pea protein } \\
\text { concentrate }\end{array}$ & 0.00 & 0.00 & 300.00 & 0.00 & 0.00 & 0.00 & 0.00 & 0.00 \\
\hline Soybean meal ${ }^{\mathrm{c}}$ & 0.00 & 0.00 & 0.00 & 300.00 & 0.00 & 0.00 & 0.00 & 0.00 \\
\hline $\begin{array}{l}\text { Soy protein } \\
\text { concentrate }\end{array}$ & 0.00 & 0.00 & 0.00 & 0.00 & 0.00 & 300.00 & 0.00 & 0.00 \\
\hline Canola meal ${ }^{\mathrm{e}}$ & 0.00 & 0.00 & 0.00 & 0.00 & 0.00 & 0.00 & 300.00 & 0.00 \\
\hline $\begin{array}{l}\text { Canola protein } \\
\text { concentrate }\end{array}$ & 0.00 & 0.00 & 0.00 & 0.00 & 0.00 & 0.00 & 0.00 & 300.00 \\
\hline $\begin{array}{l}\text { Nova Scotia fish } \\
\text { meal }^{\mathrm{g}}\end{array}$ & 390.00 & 387.10 & 275.41 & 299.64 & 0.00 & 0.00 & 0.00 & 0.00 \\
\hline West coast fish meal ${ }^{\mathrm{h}}$ & 0.00 & 0.00 & 0.00 & 0.00 & 400.00 & 217.17 & 452.80 & 407.83 \\
\hline Meat and bone meal ${ }^{1}$ & 224.80 & 0.00 & 56.24 & 45.83 & 304.00 & 89.63 & 0.00 & 0.00 \\
\hline Fish oil & 141.34 & 156.31 & 122.03 & 146.54 & 120.50 & 169.86 & 83.60 & 74.28 \\
\hline Alpha-cellulose $\mathrm{e}^{\mathrm{k}}$ & 117.94 & 0.00 & 67.63 & 90.19 & 57.80 & 103.33 & 44.20 & 95.84 \\
\hline Wheat flour ${ }^{1}$ & 100.00 & 47.56 & 100.00 & 100.00 & 100.00 & 100.00 & 100.00 & 100.00 \\
\hline Corn gluten meal & 11.16 & 95.04 & 62.70 & 2.63 & 4.75 & 0.00 & 0.00 & 0.00 \\
\hline Vitamin premix ${ }^{m}$ & 4.75 & 4.75 & 4.75 & 4.75 & 4.75 & 4.75 & 4.75 & 4.75 \\
\hline Mineral premix ${ }^{n}$ & 4.75 & 4.75 & 4.75 & 4.75 & 4.75 & 4.75 & 4.75 & 4.75 \\
\hline Choline chloride $^{\circ}$ & 4.00 & 4.00 & 4.00 & 4.00 & 4.00 & 4.00 & 4.00 & 4.00 \\
\hline DL-Methionine $\mathrm{p}^{\mathrm{p}}$ & 0.75 & 0.00 & 2.00 & 1.17 & 1.80 & 3.02 & 0.20 & 1.42 \\
\hline$L-L y \sin e^{q}$ & 0.00 & 0.00 & 0.00 & 0.00 & 1.90 & 2.99 & 5.20 & 6.63 \\
\hline Vitamin $C^{r}$ & 0.50 & 0.50 & 0.50 & 0.50 & 0.50 & 0.50 & 0.50 & 0.50 \\
\hline
\end{tabular}

*FM diet fed as control with PM, PPC and SBM.

\% FM diet fed as control with SPC, CM and CPC.

a Yellow field pea, CDC Mozart; Crop Development Centre, Saskatoon, SK, Canada.

b Pea protein concentrate, yellow field pea, prestige protein; Parrheim Foods, Saskatoon, SK, Canada.

c Soybean meal; Federated Cooperatives Limited, Saskatoon, SK, Canada.

d Soycomil@K; ADM Specialty Ingredients (Europe) BV, Koog aan de Zaan, The Netherlands.

e Canola Meal-35; Federated Co-Operative Ltd., Saskatoon, SK, Canada.

f Can Pro IP; CanPro Ingredients Ltd., Saskatoon, SK, Canada.

g Nova Scotia herring meal; Shur-Gain Aquaculture, Truro, NS, Canada.

h West Coast Fish Meal; Federated Co-Operative Ltd., Saskatoon, SK, Canada.

i Saskatoon Processing Co.; Saskatoon Processing Co., Saskatoon, SK. Canada.

j Danish Fish Oil; FF of Denmark, Skagen, Denmark.

k Solka-floc®, 200 FCC; International fiber corporation, North Tonawanda, NY, USA.

l Robin Hood All-Purpose Flour; Robin Hood Multifoods Corporation, Markham, ON, Canada.

m DL-methionine, feed grade. Degussa Corporation, Theodore, AL, USA.

n Vitamin premix, commercial (EWOS FISH-STR VIT PX, Surrey, BC; closed formulation), formulated to meet the requirements of juvenile rainbow trout; BASF Canada, Surrey, BC, Canada.

o $60 \%$ Choline Chloride; Chinook Grouplimited Partnership, Sombra, ON, Canada.

p DL-methionine, feed grade. Degussa Corporation, Theodore, AL, USA.

q L-Lysine, monohydrochloride; Ajinomoto Heartland LLC, Eddyville, IA, USA.

rAscorbic acid, pharmaceutical grade; NOW Foods, Bloomingdale, IL, USA. 


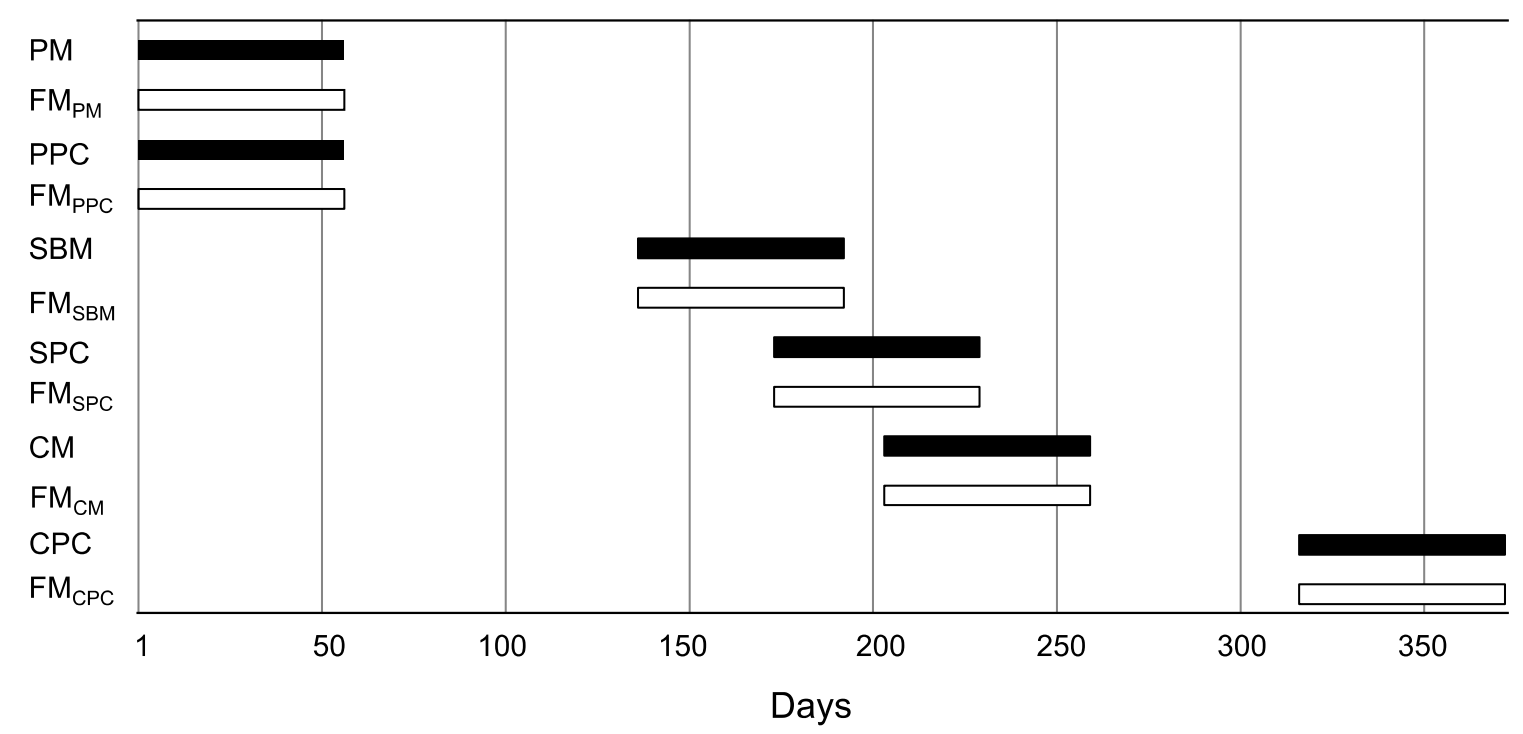

Figure 4.1 Timing of diet trials. Horizontal bars indicate the 8 -week period for each stage of the experiment. Experimental diets (black bars) containing plant ingredients were each conducted concurrently with an FM control diet group (white bars). Three fish from each of three tanks were collected for each diet, resulting in nine fish representing each diet group. 


\subsubsection{Sample collection and DNA extraction}

For each trial, after the feeding period of 8 weeks, nine fish from the experimental diet group and nine fish from the control group were selected randomly and euthanized (three fish from each of three tanks for each group). One fish from $\mathrm{FM}_{\mathrm{SPC}}$ had no intestinal contents and was excluded from the study. Distal gut contents were collected as described previously (Mansfield, et al. 2010).

Briefly, the gastrointestinal tract was dissected and the distal gut contents were squeezed out into a sterile tube and held on ice prior to storage at $-80{ }^{\circ} \mathrm{C}$ until DNA extraction. Total genomic DNA was extracted from the distal gut contents of individual fish using the QIAamp ${ }^{\circledR}$ DNA Stool Mini Kit (Qiagen Inc., Mississauga, ON).

\subsubsection{I6S rRNA PCR-DGGE and cluster analysis}

PCR was performed with universal primers F-968-GC and R-1401 flanking the V6-V8 region of the 16S rRNA gene (Nubel, et al. 1996) as described previously (Konstantinov, et al. 2003). PCR amplicon separation was done on an $8 \%$ polyacrylamide gel with a gradient of $45-60 \%$ urea as described previously (Janczyk, et al. 2007). Marker lanes were run in the outermost lanes of each gel. DNA size ladders were composed of 16S rRNA V6-V8 PCR products of bacterial isolates and clones from the hind gut of rainbow trout. Gels were stained SYBR® Gold Nucleic Acid Gel Stain (Invitrogen Canada, Burlington, ON) and visualized and photographed under ultraviolet light. 
DGGE fingerprints were normalized and analyzed using GelCompar II V6.1 software (Applied Maths Inc., Austin, TX). Percent similarity indices of the fingerprints were calculated using the Pearson product-moment correlation. Dendrograms of the fingerprints were built using the calculated Pearson correlation un-weighted pair group method algorithm (UPGMA).

\subsubsection{Sample preparation for pyrosequencing}

For each sample of intestinal contents, cpn60 UT PCR product libraries were generated using primers and PCR protocols described previously (Schellenberg, et al. 2011a). Both forward and reverse primers were modified at the 5' end with one of 14 unique multiplexing identification (MID) tags. Samples were combined into eight pools of 14 samples and sequenced in eight regions of a 16 region pico titre plate using the Roche 454 sequencing platform with Titanium chemistry (National Research Council Plant Biotechnology Institute, Saskatoon, SK).

\subsubsection{Sequence data analysis}

Pyrosequencing data was processed using the default on-rig procedures from 454/Roche (Brandford, CT) and filter-passing reads were binned by MID identity. Reads with a recognizable MID were partitioned using the SFFtools from Roche/454. Subpopulations of reads exhibiting MID degradation were identifiable using custom PERL scripts. Identification of degraded MID was possible through the identification of the cpn60 UT primer site and extraction of 
the sequence immediately 5' of the cpn60 UT primer. The sequence immediately proximal to the cpn60 UT primer $(<10 \mathrm{bps})$ is a perfect suffix of the MID sequence and thus was traced to its corresponding MID given the set of MIDs loaded in the same physical region. Operational taxonomic units (OTU) were assembled from the entire dataset (108 individual fish profiles) using the cDNA mode for newbler, an overlap MinMatchIdentity of $91 \%$, and overlap MinMatchLength of 137 (or at least 25\% of the cpn60 UT) (Schellenberg, et al. 2009). Nearest neighbours for all OTU were identified by comparison to a reference sequence database using a combination of BLASTn and SmithWaterman algorithms (wateredBLAST) as described previously (Schellenberg, et al. 2009). The reference sequence database was cpnDB_nr (retrieved from www.cpndb.ca, 2011-04-03) (Hill, et al. 2004) supplemented with cpn60 UT sequences previously derived from the trout intestinal microbiota (Mansfield, et al. 2010). Only OTU with $>55 \%$ identity to a reference sequence over at least $150 \mathrm{bp}$ were retained. Sequences with $<55 \%$ identity to the reference database were determined to be non-cpn60 based on manual examination of sequence similarity results, and were discarded. Rarefaction curves, bias-corrected Chao 1 richness and Simpson's index of diversity were calculated based on assembled OTU using MOTHUR (Schloss, et al. 2009).

Intestinal microbial profiles from the various experimental and control groups were compared using cluster analysis and principal coordinates analys is (PCoA) based on the distance metric in Fast UniFrac, incorporating sequence frequencies (weighted UniFrac) (Hamady, et al. 2010). Input phylogenetic trees 
for UniFrac were generated from the appropriate collection of nearest neighbour sequences using DNADIST and NEIGHBOR programs in PHYLIP (Phylogeny Interference Package, version 3.5c). A Methanosarcina acetivorans cpn60 sequence was used as an out group to root the tree, as required by UniFrac. 


\subsection{Results}

\subsubsection{Sequence data and ecological metrics}

The fish consumed all diets readily and their specific growth rates were greater than $1 \%$ per day for fish on all dietary treatments. A total of 99,568 reads were obtained after quality control and MID identification, with an average of 1,660 $( \pm 1386)$ reads per sample $(\mathrm{n}=99$ fish $)$. Eight libraries were omitted from the analysis due to small library size (fewer than 100 reads), including one from each of the PPC and CM experimental diet groups and three each from $\mathrm{FM}_{\mathrm{CM}}$ and $\mathrm{FM}_{\mathrm{CPC}}$. A total of 965 operational taxonomic units (OTU) were assembled from the combined data and $94 \%$ of these sequences were at least $80 \%$ identical to a reference database sequence. Nearest neighbours and abundance values for all OTU in all samples are given in Supplemental Table 1. OTU abundances were scaled to the median library size of 1,386 reads.

Rarefaction curves were plotted for each diet group (Figure 4.2). The Good's coverage estimator for experimental diets ranged from 0.945 (CM) to 0.961 (SBM). The Good's coverage estimator for the FM control diet replicates ranged from 0.948 for $\mathrm{FM}_{\mathrm{PM}}$ and $\mathrm{FM}_{\mathrm{CM}}$ to 0.978 for FMSBM. Chao 1 richness of microbiota associated with the experimental diets was consistently higher than the corresponding FM control diets (Figure 4.3A). A similar, non-significant trend was seen for Simpson's diversity index (Figure $4.3 \mathrm{~B}$ ) with experimental diet microbiota having higher diversity than the FM controls. A non-significant 
trend of decreasing diversity of the intestinal microbiota in FM control diet fish was observed over the course of the experiment ( 0.9 for $\mathrm{FM}_{\mathrm{PM}}$ to 0.7 for $\mathrm{FM}_{\mathrm{CPC}}$ ).

\subsubsection{Changes in FM control diet associated microbiota}

The distal gut intestinal microbiota structure of rainbow trout fed with different diets was examined by DGGE of $16 \mathrm{~S}$ rRNA PCR products. Reproducibility of PCR amplification and DGGE was tested and Pearson similarity coefficients for 18 pairs of replicates were at least $95 \%$ (data not shown). DGGE fingerprints for all individual fish are shown in Figures 4.4 and 4.5 . 

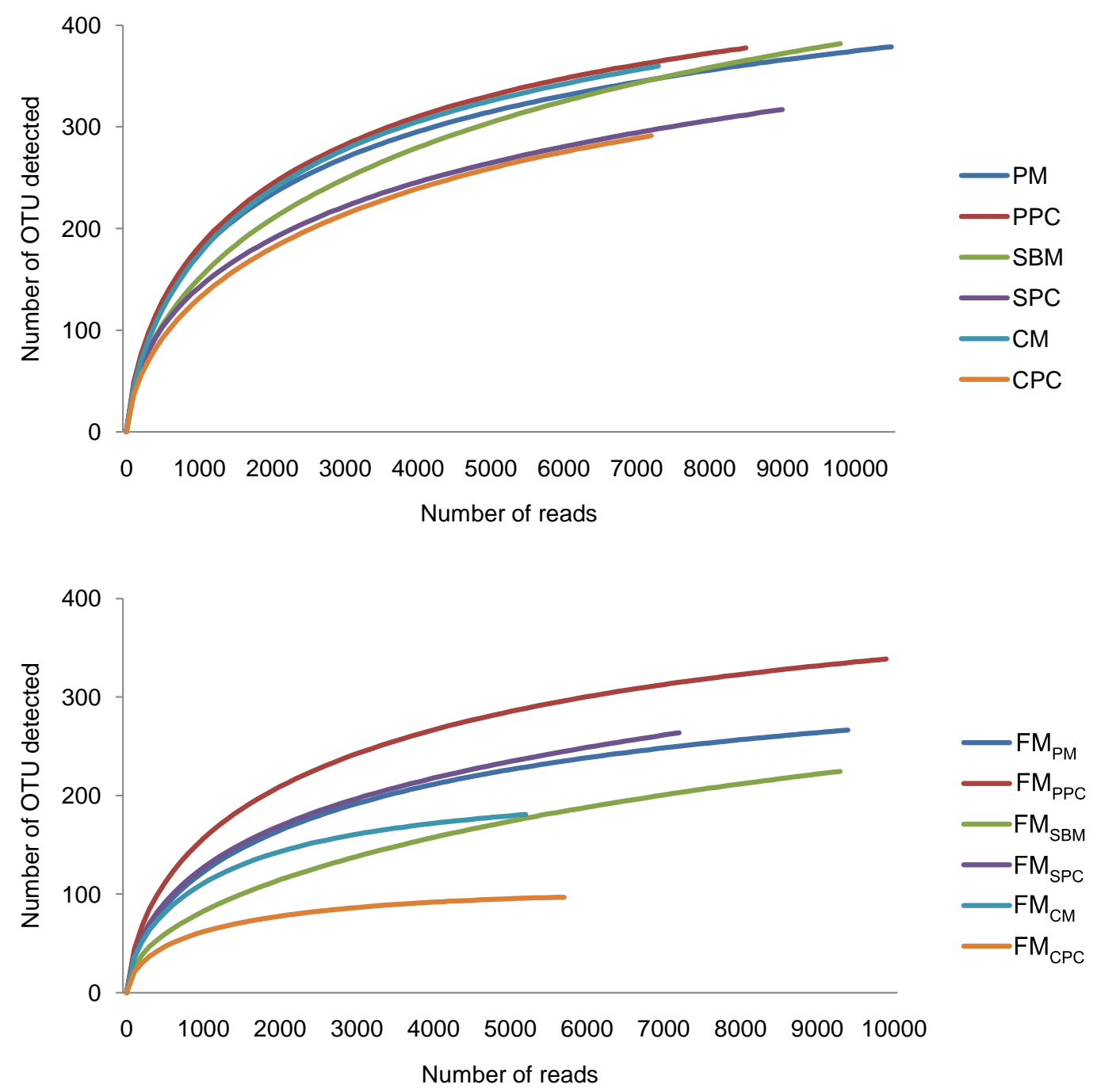

Figure 4.2 Rarefaction curves of cpn60 UT amplicon libraries of intestinal microbiota of fish fed with different plant-based diets (upper panel) and FM control diets (lower panel). 
A.

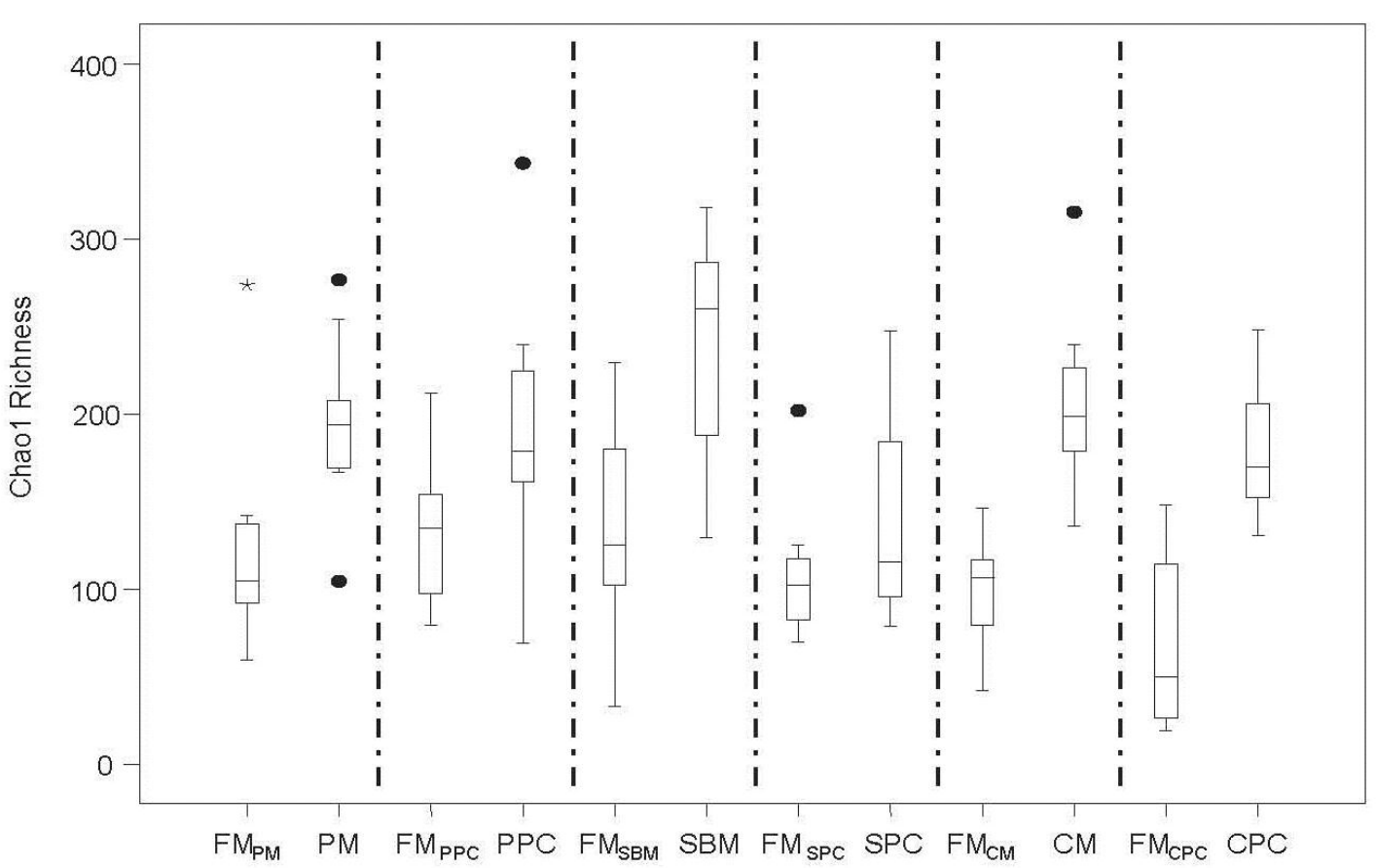

B.

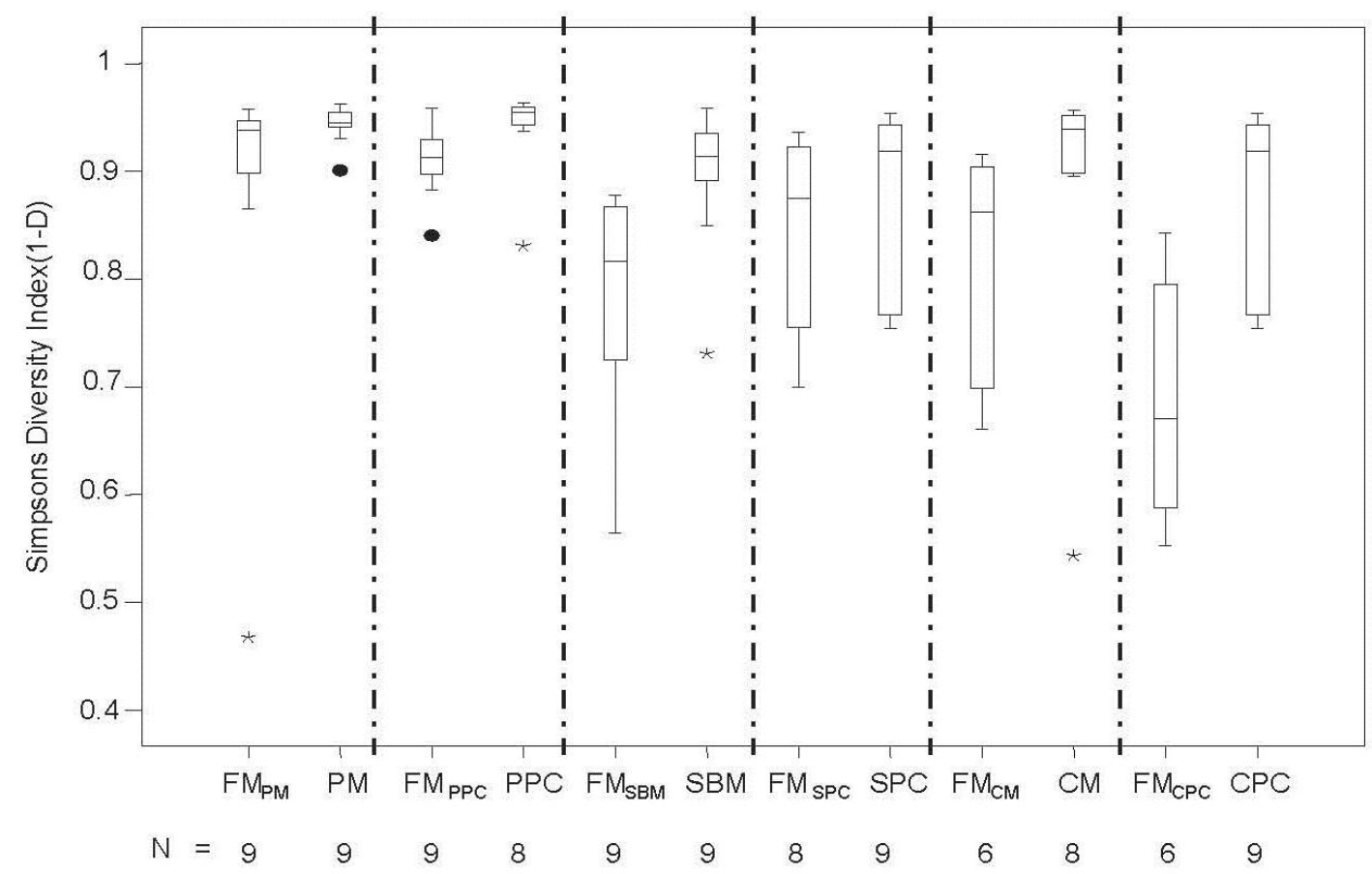

Figure 4.3 Box plots (median, quartiles and range values) of (A) Chao 1 richness and (B) Simpson's diversity index of intestinal microbiota of fish fed with different diets. $\mathrm{N}$ indicates number of fish included in analysis. 

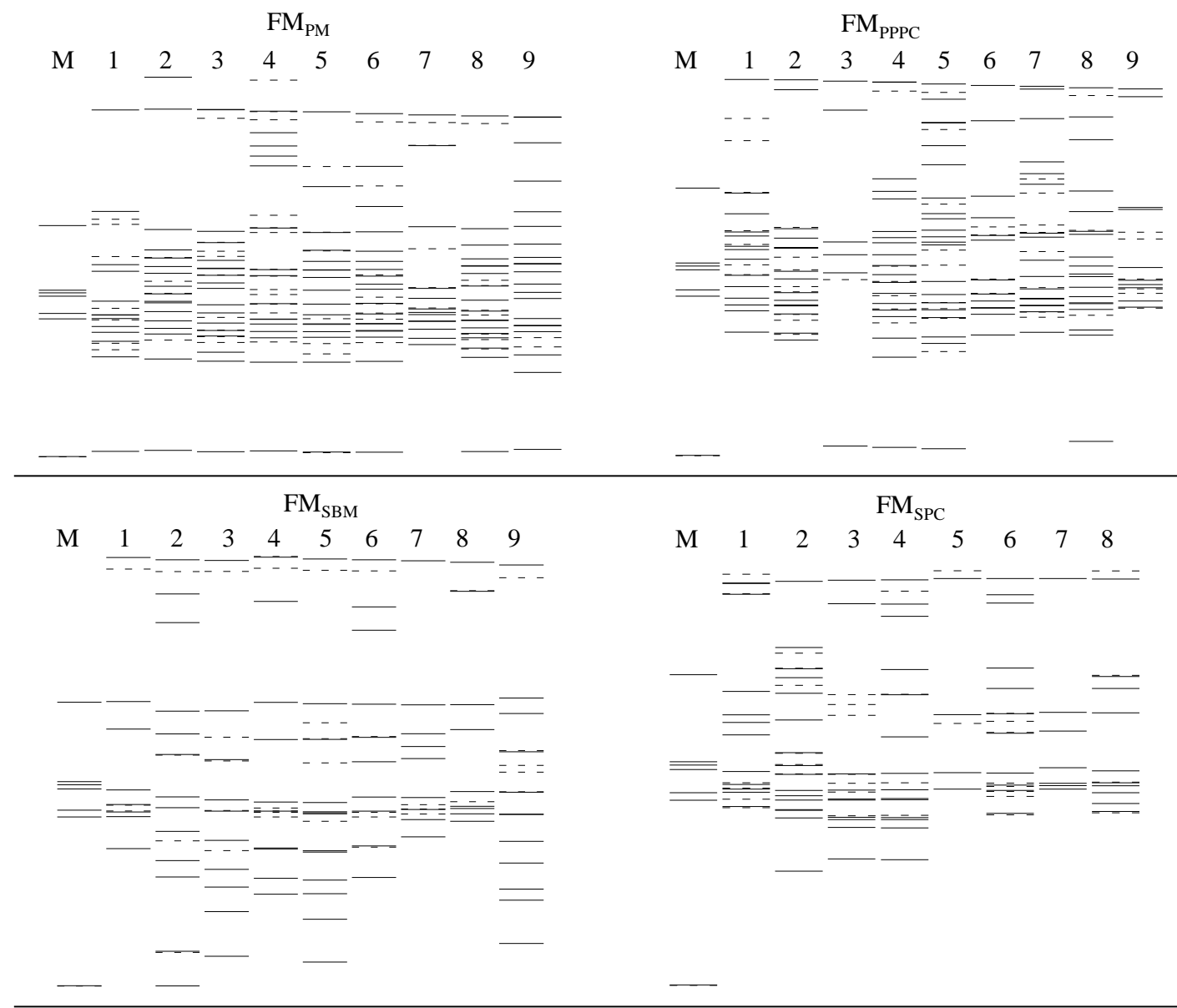

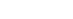

\begin{tabular}{lllllllll}
\multicolumn{1}{c}{$\mathrm{FM}_{\mathrm{SPC}}$} \\
$\mathrm{M}$ & 1 & 2 & 3 & 4 & 5 & 6 & 7 & 8
\end{tabular}

$\mathrm{FM}_{\mathrm{CM}}$

$\mathrm{FM}_{\mathrm{CPC}}$
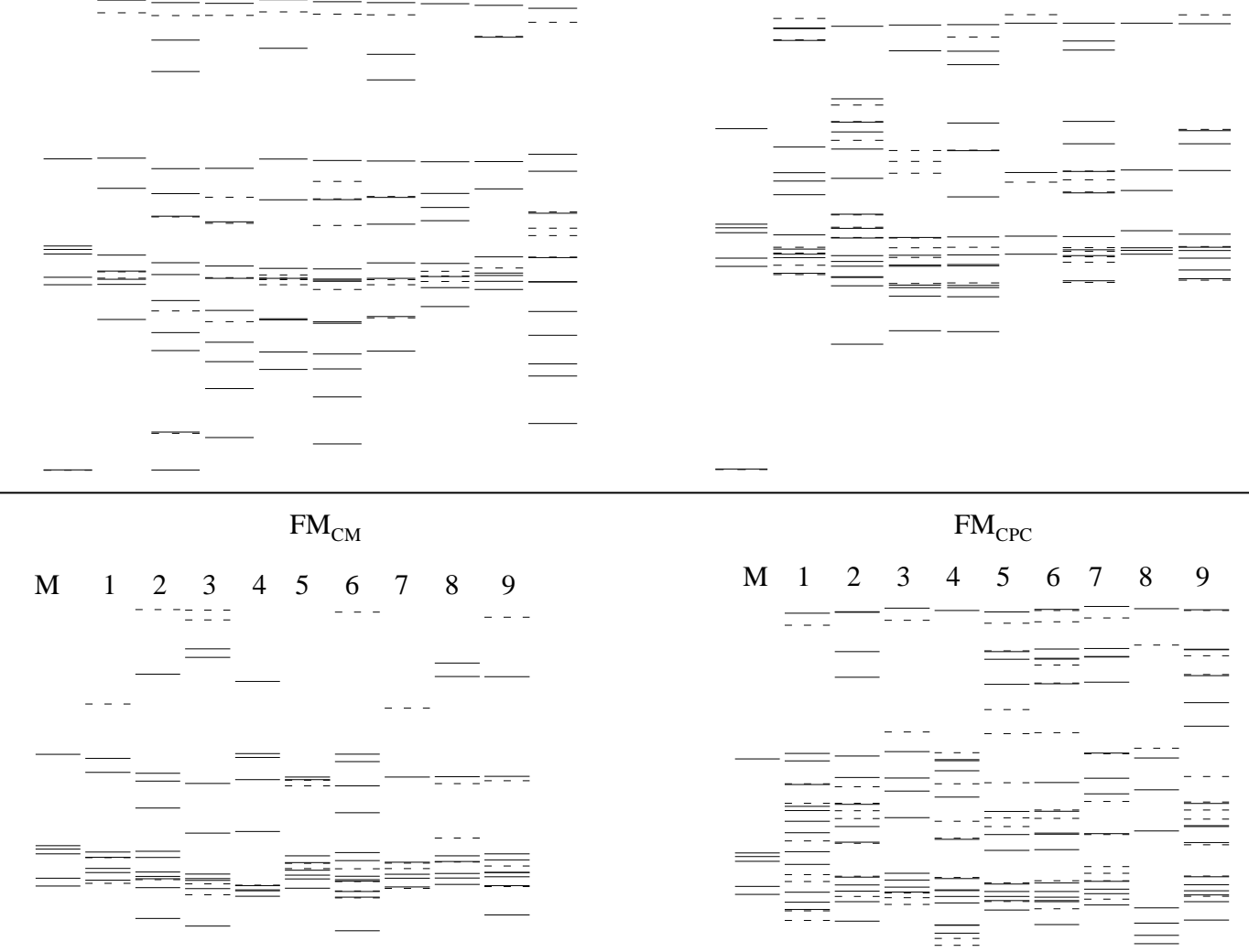

(1)

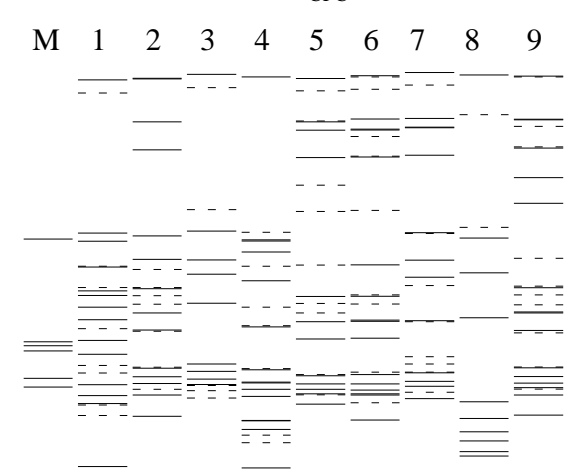

Figure 4.4 DGGE fingerprints of distal gut contents of individual fish fed with the FM control diet during the course of experiment. M indicates marker lane. 
PPC
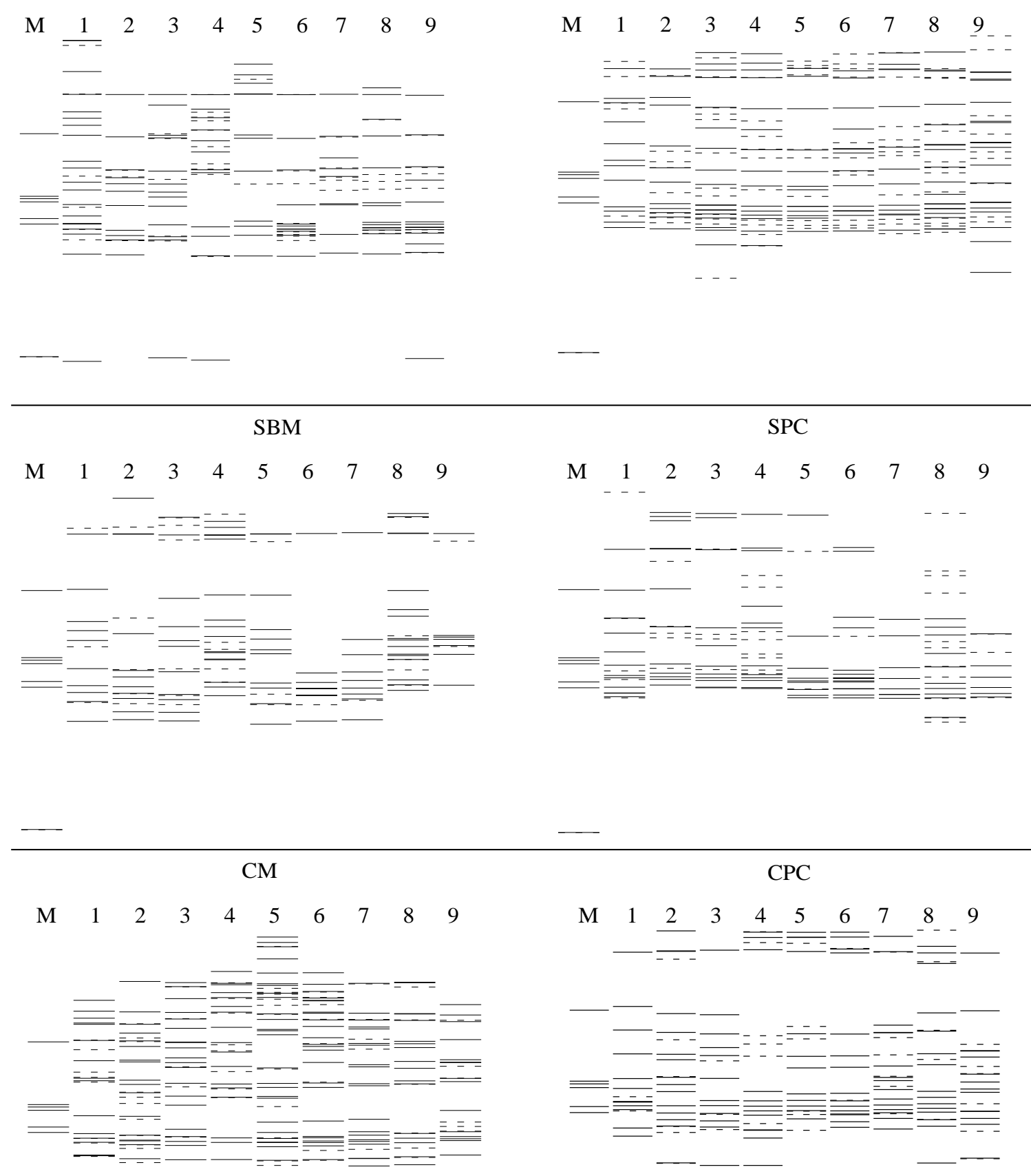

Figure 4.5 DGGE fingerprints of distal gut contents of individual fish fed with experimental diets. $M$ indicates marker lane. 
Six replicates of the FM control diet $\left(n=8\right.$ for $\mathrm{FM}_{\mathrm{SPC}}$ and $\mathrm{n}=9$ for all others) were available for analysis. UPGMA analysis based on the DGGE profiles of fish fed the FM control diet showed clear clustering of individual fish according to the six experimental replicates suggesting the structure of intestinal microbiota associated with the FM control diet changed over the course of the experiment (Figure 4.6).

A similar trend was observed in the pyrosequencing data. Clustering of FM control diet samples and PCA using the unweighted UniFrac distance metric indicated that FM control diet fish associated intestinal microbiota in the $\mathrm{FM}_{\mathrm{SBM}}$, $\mathrm{FM}_{\mathrm{CM}}$ and $\mathrm{FM}_{\mathrm{SPC}}$ experiments were more closely related to each other than they were to fish in the $F M_{C P C}, F M_{P M}$ or $F M_{P P C}$ trials (Figure 4.7). Also, $F M_{P P C}$ and $\mathrm{FM}_{\mathrm{PM}}$ clustered together in both analyses, reflecting the temporal relationships of the experiments (Figure 4.1). 


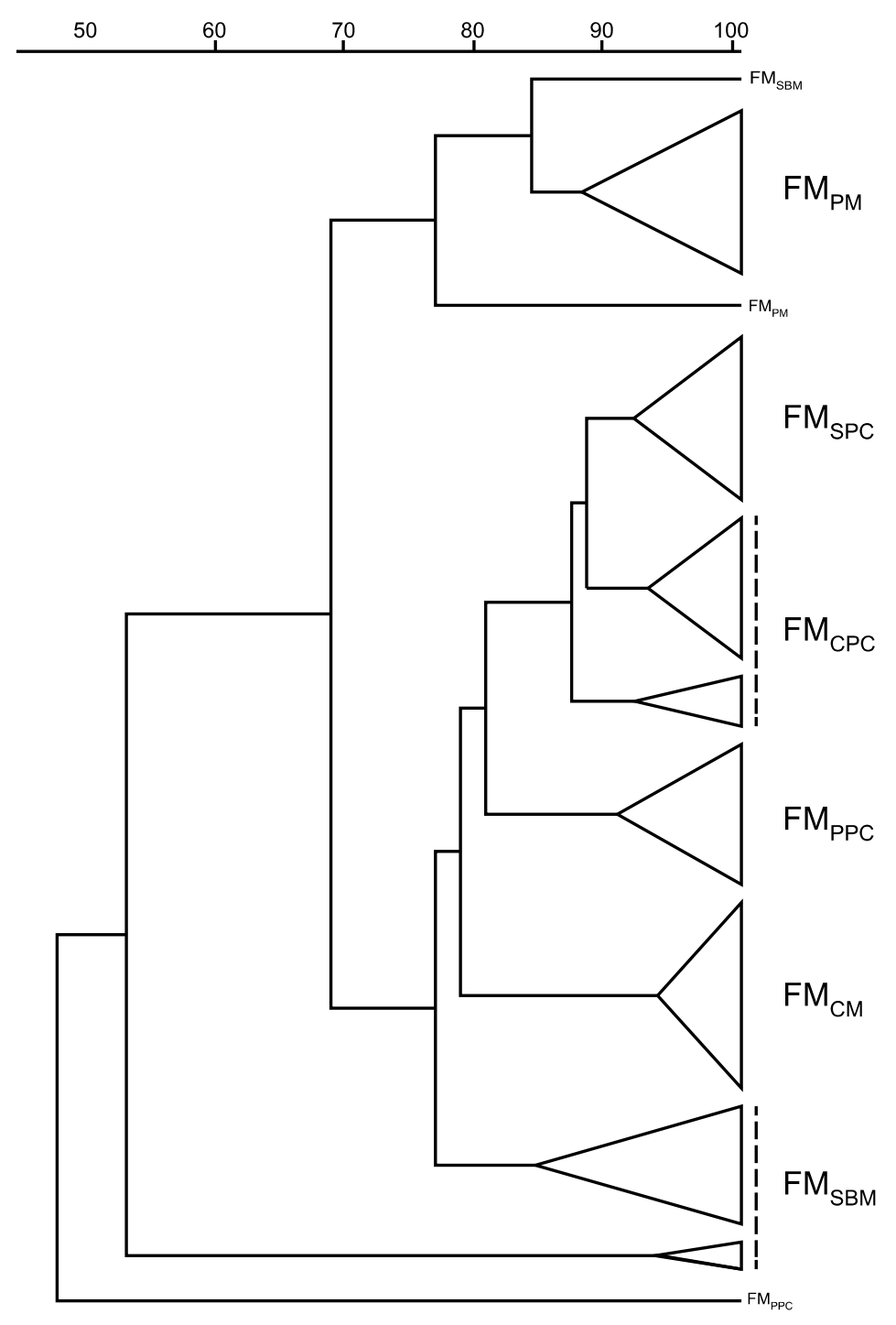

Figure 4.6 16S rRNA gene PCR-DGGE analysis of the distal gut content samples obtained from fish fed with the FM control diet over the course of the experiment. The dendrogram was collapsed at the lowest common node for each FM control diet group. The scale indicates percent similarity based on Pearson product-moment correlation. 
A.

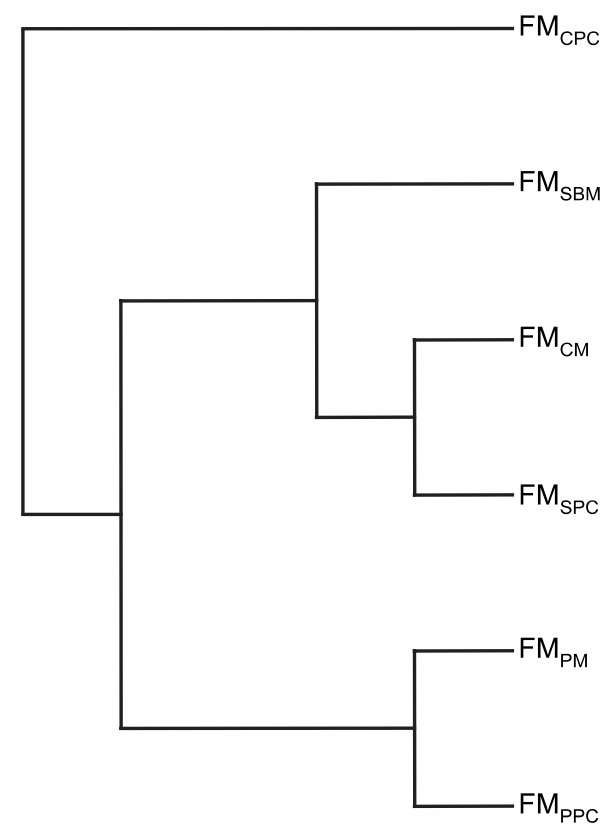

B.

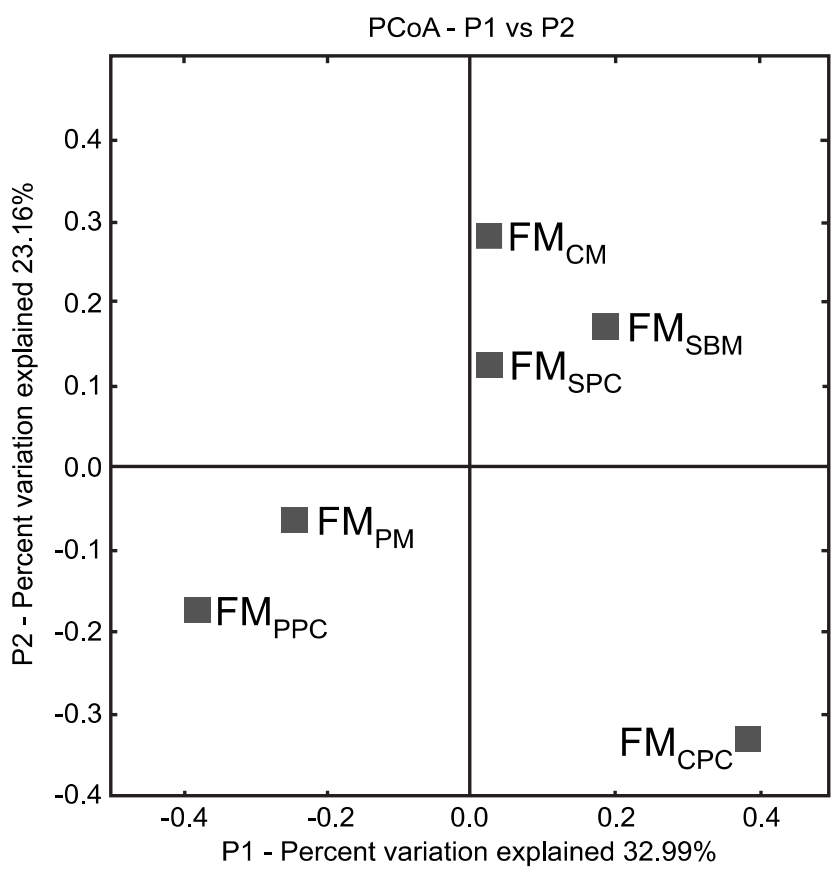

Figure 4.7 Relationships of FM control diet associated profiles determined by cpn60 UT pyrosequencing. (A) UPGMA based cluster analysis and (B) principal coordinates analysis of profiles based on unweighted UniFrac distance. 


\subsubsection{Effects of plant ingredient inclusion on intestinal microbiota}

As anticipated, most of the OTU associated with the intestinal microbiota of rainbow trout were assigned to the Proteobacteria (609 OTU) and Firmicutes (197 OTU). The remaining OTU were largely Actinobacteria (96 OTU) and Bacteroidetes (22 OTU). It was observed that the richness and abundance of OTU varied from diet to diet, but irrespective of the diet Proteobacteria and Firmicutes were most frequently detected in all fish intestinal microbiota samples. The most prevalent (detected in $>70 \%$ of samples) OTU observed in intestinal microbiota associated with the experimental diets included both Firmicutes and Proteobacteria (Supplemental Table 1). Nine of the 11 most prevalent Firmicutes OTU had Weissella paramesenteroides as their nearest neighbour with 82.5-85.3 \% identity. Prevalent Proteobacteria OTU were identified as Acidovorax facilis (97.8\% identity), Citrobacter freundii (99.5\%), Enterobacter amnigenus (99.5\%) and Alicycliphilus dentrificans (90.3\%). Only three OTU were prevalent in more than $70 \%$ of the fish intestinal microbiota samples from the FM control groups, two of which were classified as Firmicutes

(84.2-85.3\% identical to Weissella paramesenteroides) and one as Proteobacteria (100\% identical to rainbow trout intestinal microbiota clone cn_0_10, 89\% identical to Shewanella oneidensis). A positive correlation between the cumulative abundance and the prevalence of the OTU in fish fed both experimental diets $(\rho \mathrm{s}=0.808, \mathrm{n}=965, \mathrm{p}<0.0001)$ and $\mathrm{FM}$ control diets $(\rho \mathrm{s}=0.935, \mathrm{n}=965, \mathrm{p}<0.0001)$ was observed, indicating some consistency in microbiota composition across individual fish (Supplemental Table 1). Total 
bacterial counts for the intestinal contents associated with fish fed with the experimental diets based on a $16 \mathrm{~S}$ rRNA universal target qPCR were $8.2 \times 10^{9} \pm$ $5.7 \times 10^{10}$ copies per gram (data not shown).

When sequence data from individual fish were pooled by diet, microbial profiles associated with the FM control diet replicates showed a trend of increasing proportions of Proteobacteria over the course of experiment, from $65 \%$ of reads in $\mathrm{FM}_{\mathrm{PM}}$ to $92 \%$ of reads in $\mathrm{FM}_{\mathrm{CPC}}$ (Figure 4.8). Decreasing Firmicutes:Proteobacteria ratios were also apparent when individual fish were considered. The median ratio of abundance of Firmicutes:Proteobacteria in FM control diets decreased from 0.4 in $\mathrm{FM}_{\mathrm{PM}}$ to 0.03 in $\mathrm{FM}_{\mathrm{CPC}}$ (Figure 4.9). 


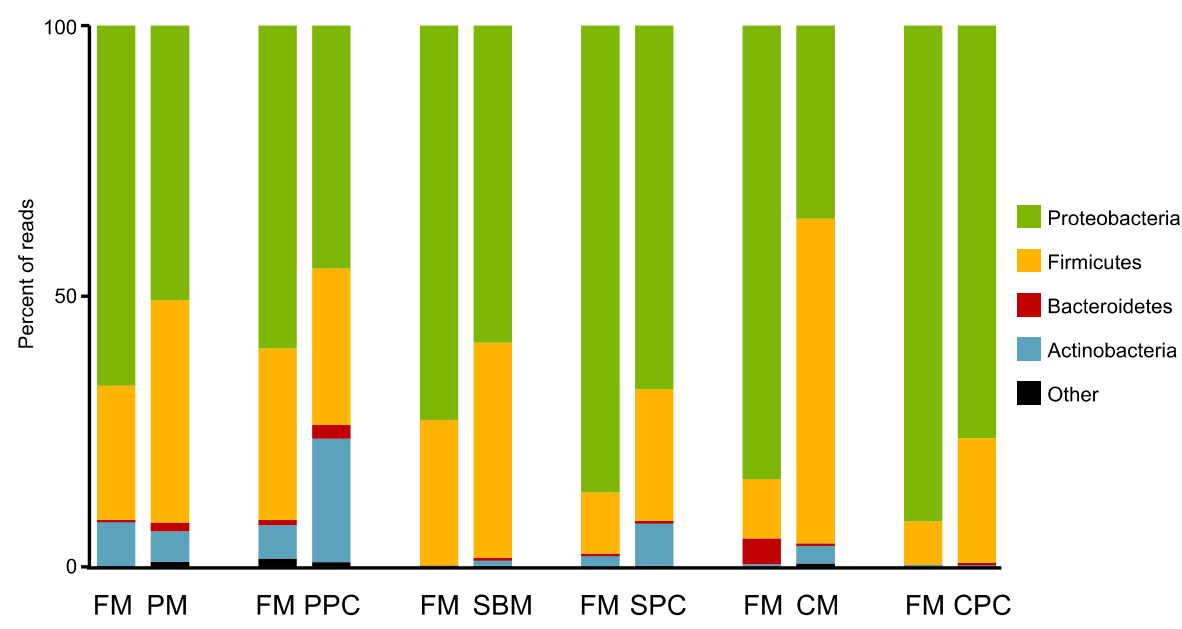

Figure 4.8 Proportional abundance of bacterial phyla represented in cpn60 UT amplicon libraries derived from the intestinal contents of rainbow trout. 


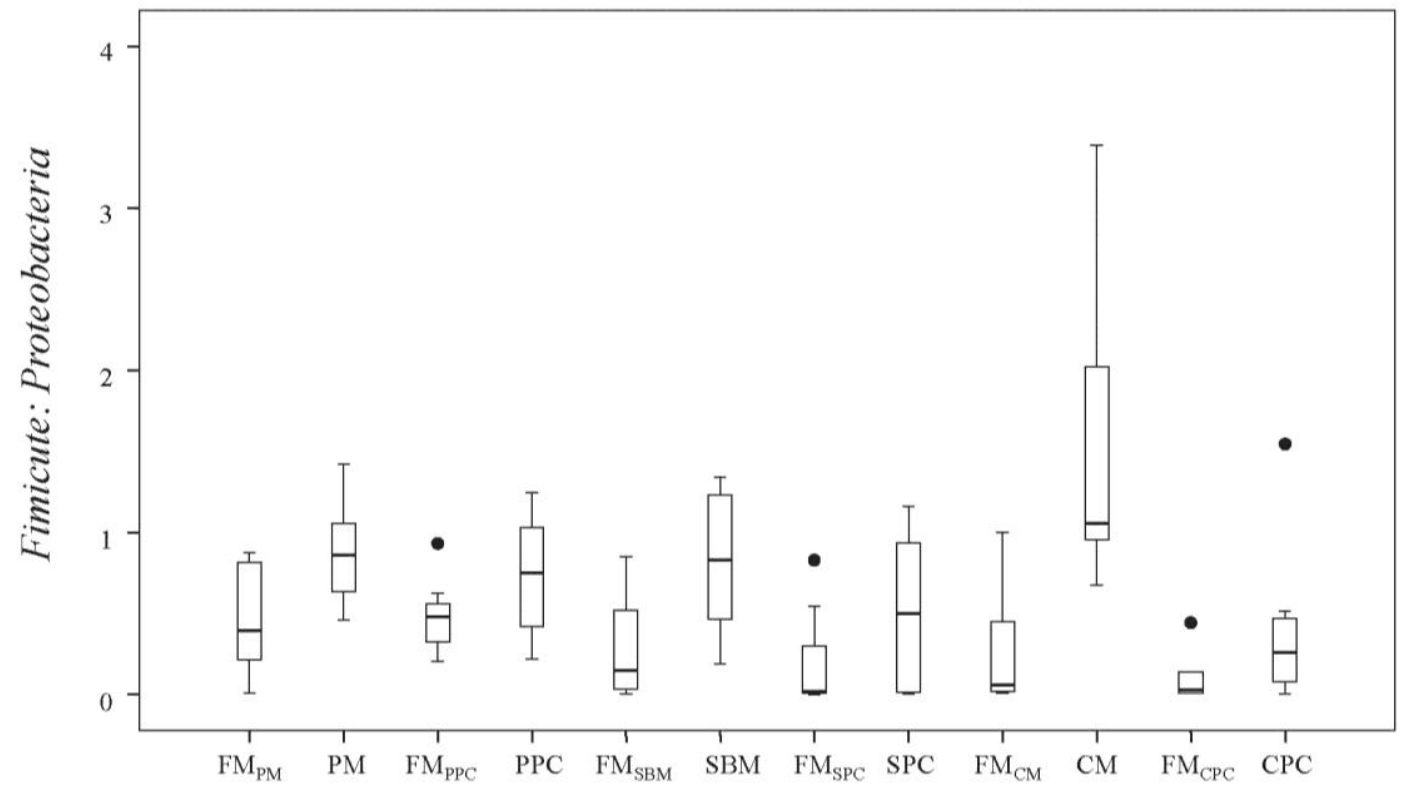

Figure 4.9 Box plots (median, quartiles and range values) of Firmicutes:Proteobacteria ratios for individual fish fed experimental and control diets. In each case, ratios are higher in the experimental (plant ingredient) diet than in the corresponding fish meal control. 

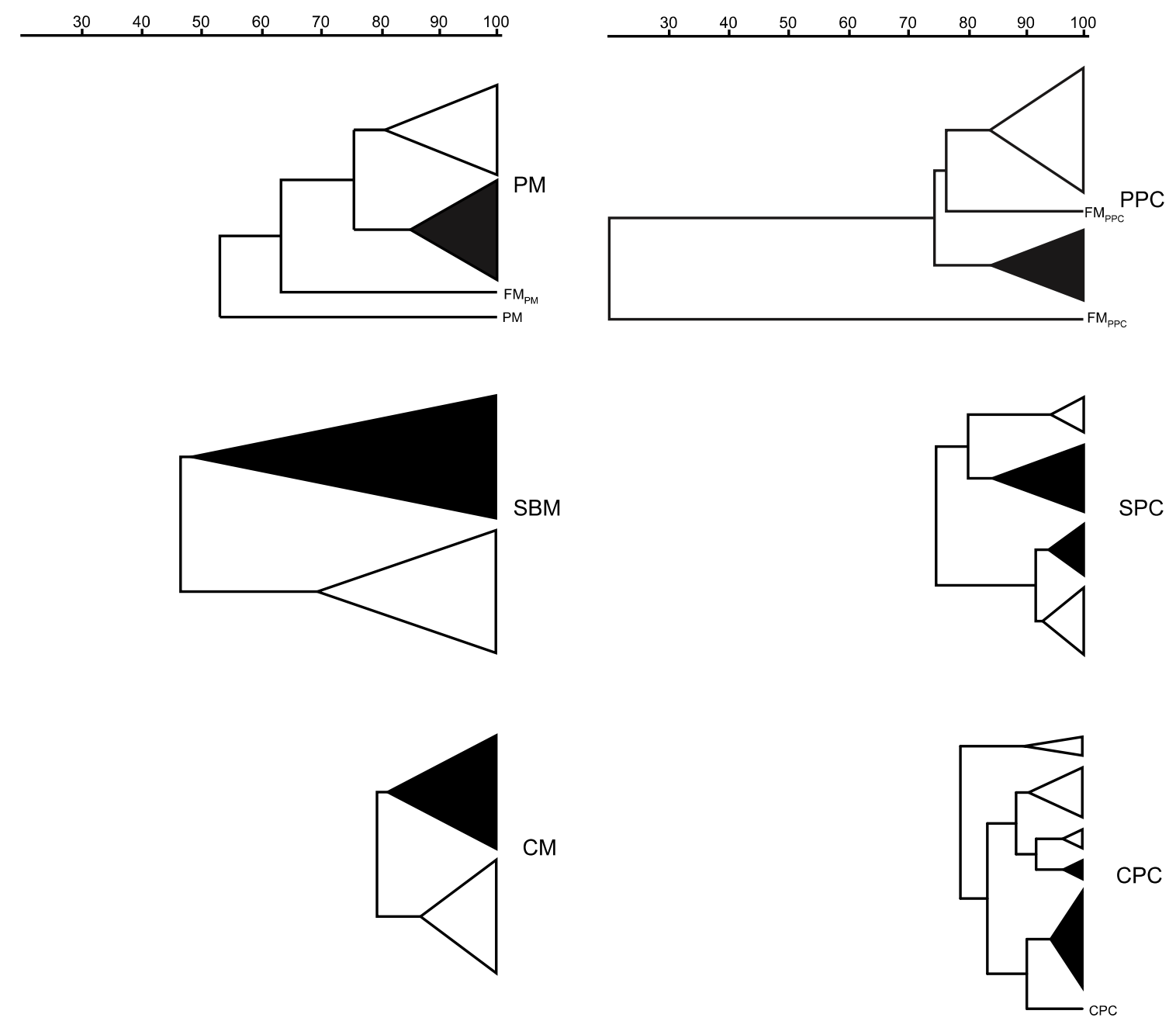

Figure 4.10 Clustering of DGGE fingerprint profiles of intestinal microbiota of rainbow trout associated with experimental diet and concurrent controls. In each dendrogram, the experimental diet profiles are indicated with black triangles, control diet profiles with white triangles. 
Since intestinal microbial profiles associated with the FM control diet replicates changed over the course of the experiment, a direct comparison of the microbial profiles associated with the experimental diets was not reasonable. Instead, we examined the differences between microbial profiles associated with the experimental diets and their concurrent FM control group. This comparison showed a consistent but non-significant trend of higher Firmicutes: Proteobacteria ratios in experimental diet profiles compared to the concurrent FM control groups (Figure 4.8). Box and whisker plots showing the trend when individual fish were considered are shown in Figure 4.9.

Differences between the microbiota associated with each experimental diet and its corresponding FM control were also observed in DGGE profiles (Figure 4.10). Minimum similarity indices between profiles from experimental diets and their concurrent controls ranged from a low of $47 \%$ for SBM to a high of $82 \%$ for CM. For each of the meal-based diets (SBM, PM and CM), the experimental diet-associated microbiota was clearly distinguishable from the FM control microbiota (Figure 4.10). DGGE profiles associated with the protein concentrate diets were generally less distinguishable from their corresponding control diet profiles (Figure 4.10). In cases where the experimental or control diet profiles formed more than one cluster (SPC and CPC, Figure 4.10), the se clusters did not correspond to the tank assignment of the fish. SBM profiles were the most diverse of the experimental diet DGGE profiles.

Weighted UniFrac cluster analysis and PCA of cpn60 based pyrosequencing profiles showed similar relationships to the $16 \mathrm{~S}$ rRNA DGGE 
analysis. Comparison of individual fish microbial profiles associated with meals and the corresponding FM control profiles showed a clustering based on the diet (Figure 4.11). However, the microbiota of fish fed with FM control diet and the concentrate diets were less distinguishable and did not form discrete clusters (Figure 4.11). 


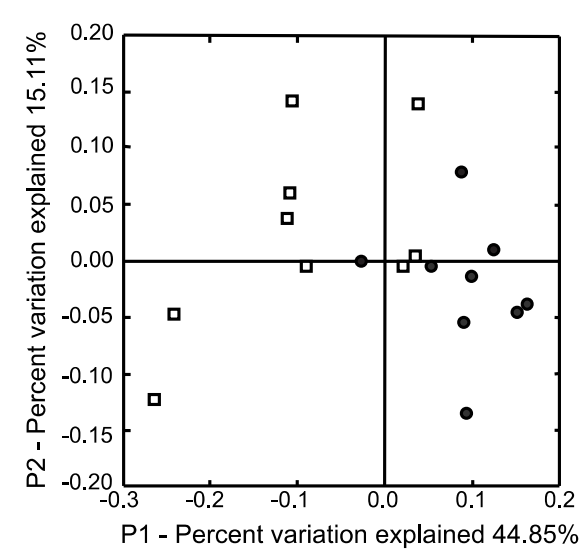

PM

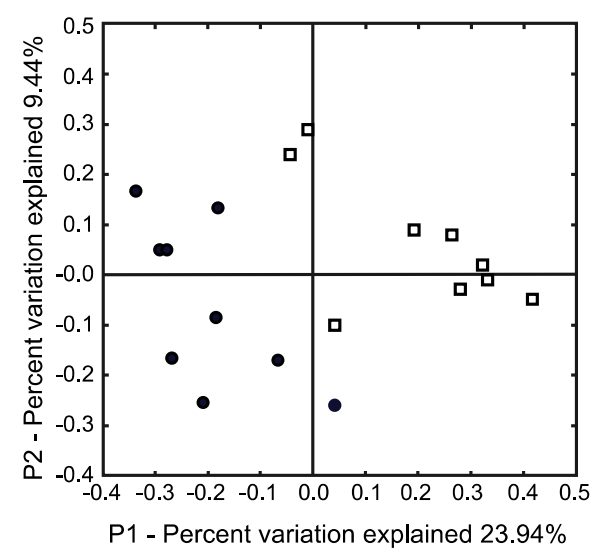

SBM

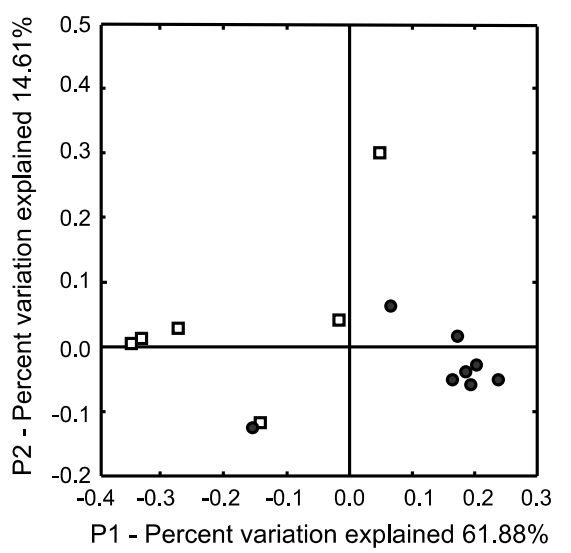

$\mathrm{CM}$

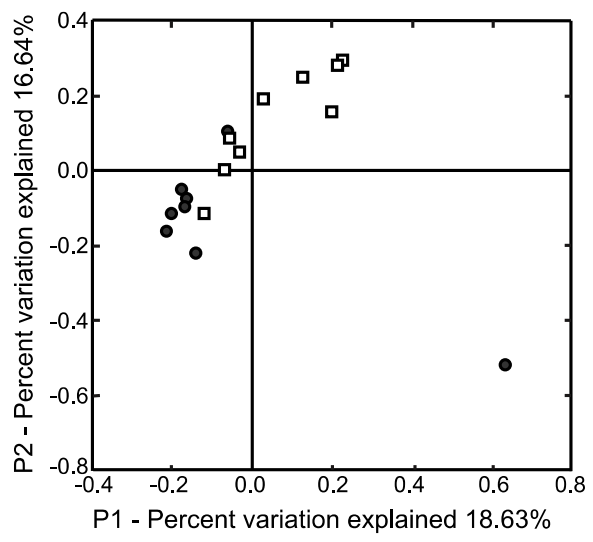

PPC
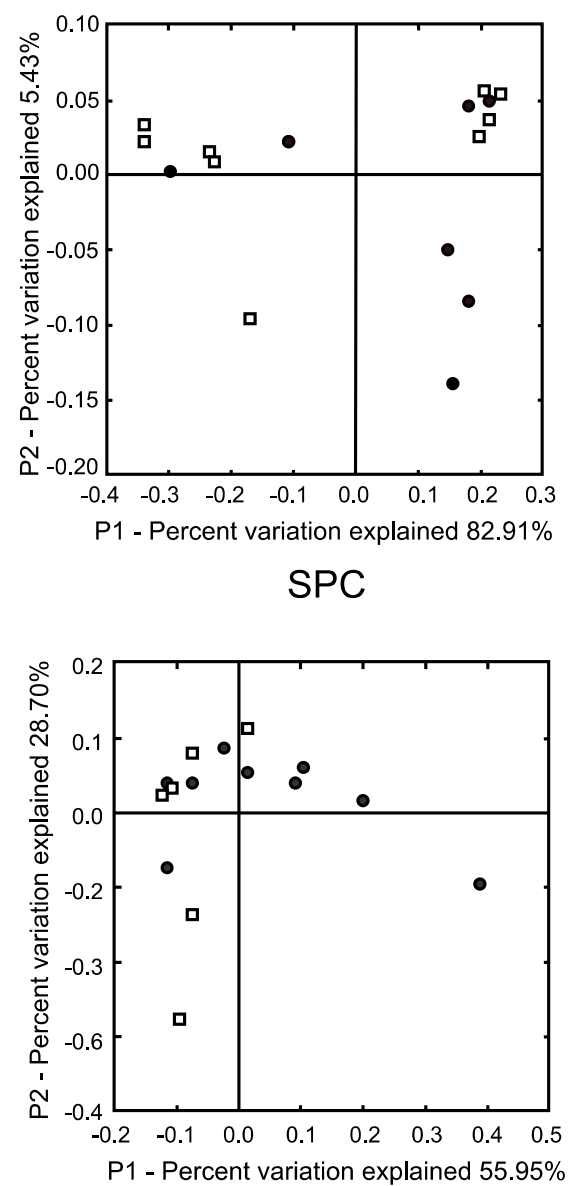

CPC

Figure 4.11 Principal coordinates analysis based on weighted UniFrac distance. White squares indicate fish fed with FM control diet and black circles indicate fish fed the experimental diet.

\subsection{Discussion}


Reliance on FM as the primary protein source for aquafeeds must inevitably decrease, due to decreased global availability of forage fish that are the source for FM production, and a corresponding increase in cost of FM (Tacon, et al. 2008). However, the replacement of fish meal with useful inclusion rates of plant ingredients generally results in decreased growth performance in carnivorous fish. One strategy to increase protein availability and reduce anti-nutritional properties of plant ingredients is to process plant meals to form protein concentrates using fractionation methods such as air classification (PPC) or aqueous extraction of protein (SPC and CPC) (Drew, et al. 2007). These methods may also remove undigestible components (non-starch polysaccharides; NSP) and antinutritional factors (Francis, et al. 2001). Previous studies in humans (Walker, et al. 2011) and pigs (Bindelle, et al. 2011) have reported that the chemical composition of the diet, particularly undigestible diet fractions such as NSP, may significantly affect the intestinal microbiome.

The intestinal microbiota plays critical roles in intestinal development, pathogen and digestion in fish (Gomez, et al. 2008; Merrifield, et al. 2010). Ideally, any manipulation of diets to increase plant ingredient content would cause minimal disruption in the structure of the microbiome or movement away from the ideal microbiome associated with a gold standard, FM diet.

Quantifying the effects of diet change on the intestinal microbiome is complicated by variation in the microbiota of individual animals. Even in animal production environments where genetically similar animals are reared in 
a common environment and consume the same diet, considerable animal-toanimal variation is consistently observed (Hill, et al. 2005b; Heikkinen, et al. 2006; Mansfield, et al. 2010). Historically, for economic and logistical reasons, pooling of samples has been a common approach applied in microbial ecology studies. However, when the abundance of specific targets is determined in the samples comprising these pools, the quantity of target detected in the pool is found to reflect the highest level of target abundance observed in any of the individuals comprising the pool (Hill, et al. 2005b; Desai, et al. 2009; Mansfield, et al. 2010). Even with an "averaging effect', of pooling, the logscale differences in target abundance between individuals lead to the abundant samples masking the contributions of samples with lower abundance. Nextgeneration sequencing methods have largely eliminated this issue, making it possible to examine sufficient numbers of individual samples to draw more robust conclusions about the population. Our study involved a comparison of pyrosequencing of cpn60 UT amplicons to the widely used 16S rRNA DGGE technique for detecting differences in the intestinal microbiome associated with different diet formulations. The resolution and information detail provided by these techniques are radically different, but they both provided a similar assessment of the degree of perturbation of the intestinal microbiome precipitated by meal and protein concentrate ingredients.

Our observation of the dominance of gamma Proteobacteria and Firmicutes (especially lactic acid bacteria) in the trout distal intestine is consistent with previous studies based on both culture-based and culture- 
independent approaches (Spanggaard, et al. 2000; Huber, et al. 2004; Kim, et al. 2007; Navarrete, et al. 2010). Although these phyla were dominant regardless of the diet, consistent, non-significant trends were observed in differences in their proportions in microbial profiles associated with experimental diets and controls. The plant ingredient diets were associated with higher Firmicutes:Proteobacteria ratios and correspondingly higher overall richness and diversity (Figure 4.8 and Figure 4.3). Lactic acid bacteria are prevalent constituents of the intestinal microbiome of many fish species (Cai, et al. 1998; Ringo, et al. 1998; Ringo, et al. 2001; Hagi, et al. 2004) and are generally considered as beneficial organis ms associated with a healthy intestinal epithelium (Dimitroglou, et al. 2009). Lactic acid bacteria have also been shown to increase the digestibility of soybean based diets (Burr, et al. 2008; Salze, et al. 2008) and are probiotic candidates (Cai, et al. 1998). It seems somewhat counterintuitive that an increase in "beneficial" taxa should be observed under conditions thought to be disadvantageous to fish health and growth. However, our view of the organis ms present is restricted to target genes that provide phylogenetic information only, not phenotypic characterization and it is well established that significant phenotypic variation occurs within defined "species" of prokaryotes (Perna, et al. 2001; Vermette, et al. 2010). In a previous study comparing FM and SBM diets, we observed similarly greater abundance of Firmicutes in the SBM fish compared to FM and this was associated with increased detection of inflammatory markers PCNA and IgM (Mansfield, et al. 2010). 
The observation that the meal diets consistently produced a microbial profile more divergent from FM than the corresponding protein concentrate ingredient diets was confirmed by two different target genes (16S rRNA and cpn60) and two different techniques (PCR-DGGE and amplicon pyrosequencing). The phenomenon was most dramatic for the SBM and SPC diets. DGGE profiles for $\mathrm{SBM}$ and $\mathrm{FM}_{\mathrm{SBM}}$ shared a minimum similarity of approximately $40 \%$ whereas the SPC diet produced profiles that were not completely distinguishable from the FM control, and shared a dramatically higher minimum similarity of $>70 \%$ (Figure 4.10). This difference was also obvious in the cpn60 UT sequence based microbial profiles. In the principal coordinates analysis, the fish fed the SBM diet form a broad but distinct cluster separated from the $\mathrm{FM}_{\mathrm{SBM}}$ fish whereas the SPC diet profiles are intermingled with the FM $_{\text {SPC }}$ profiles (Figure 4.11). Soybean meal has been studied extensively as a replacement for fish meal but its use has been limited by associations with intestinal inflammation and epithelial damage in salmonids at high inclusion rates (Krogdahl, et al. 2003; Heikkinen, et al. 2006; Bakke-McKellep, et al. 2007). Processing of SBM by heat treatment, aqueous or solvent extraction and fractionation to produce SPC reduces the antinutritional components of the ingredient and increases the availability of proteins and palatability (Anderson, et al. 1995). Our results suggest that processing of $\mathrm{SBM}$ also minimizes perturbation of the intestinal microbiome. The differences between meal and protein concentrate for pea and canola-based diets were subtler but still consistent, indicating that purified plant proteins can 
be used in fish diets at a $30 \%$ inclusion rate to support an intestinal microbiome less divergent from an ideal FM diet.

Another significant finding in this study was the observed changes in the FM control diet-associated microbiome. FM diet microbial profiles determined by both $16 \mathrm{~S}$ rRNA DGGE (Figure 4.6) and cpn60 amplicon sequencing (Figure 4.7) clearly clustered according to when the diets were fed during the trial (Figure 4.1). Changes that contributed to this clustering included reduced diversity, richness and a gradual decrease in the Firmicutes:Proteobacteria ratio over the 12 month course the trial. There have been few studies of long-term changes in the intestinal microbiome of aquaculture fish (Holben, et al. 2002; Hagi, et al. 2004). A short-term transition in the microbiome structure might be anticipated as a result of transfer of these fish from the lake pen environment where they would have access to a variety of food sources in addition to commercial feed, to the recirculating aquaculture facility. A comparison of "recently caught" and domesticated zebra fish suggests that the differences would largely consist of a change in relative abundances of constituents of a core microbiome for this species (Roeselers, et al. 2011). However, the longer term changes we observed were somewhat surprising and suggest that factors such as stress, age, developmental stage and the selective pressure of the maintenance diet continue to affect the microbiome over the long term. Obviously, this type of long-term microbial succession should be a critical consideration in experimental design when evaluating diets and ingredients. It also has implications for industry strategies such as the shift of carnivorous fish 
to a fish meal diet shortly before harvest in order to improve taste and nutritional quality according to consumer preference (Powell. 2003).

Our observations support the hypothesis that changes to the structure of the intestinal microbiome are at least partially responsible for the negative impacts of plant protein meal ingredients incorporated at high inclusion rates on fish growth and health. Taken together, our data show that the use of the protein concentrate forms of these ingredients reduces the impact of the ingredients on intestinal microbiome structure. The knowledge gathered from this study is not just relevant to salmonids, but will also be useful for other carnivorous fish, helping the aquaculture industry to reduce its dependence on fish meal. 


\section{Chapter 5 Application of quantitative real-time PCR for validation}

of metagenomic sequencing libraries and investigation of the reproducibility of plant ingredient effects on the rainbow trout intestinal microbiome.

Chapter 5 is a part of a multi-disciplinary, collaborative study. Diet formulations described in Table 5.1 were done by Stephanie Collins. All other data presented in this chapter are the work of the thesis author. 


\subsection{Abstract:}

High throughput pyrosequencing of amplicon libraries has become a common technique for characterisation of the intestinal microbiota of animals. However, the degree to which this technique is quantitative has not been widely investigated. This chapter describes the use of quantitative real-time PCR (qRT-PCR) based on the cpn60 gene for validation of pyrosequencing data from the intestinal microbiome of rainbow trout (Onchorhynchus mykiss) fed with different plant ingredient based diets. qRT-PCR and 16S rRNA denaturing gradient gel electrophoresis (DGGE) were also applied in an investigation of the reproducibility of previously observed effects of plant ingredients on the structure of the trout distal gut microbiome. Target copy numbers detected by qRT-PCR were not generally consistent with numbers of sequence reads detected in cpn60 amplicon libraries generated from the same samples. Furthermore, although DGGE analys is of individual fish confirmed that plant ingredients were reproducibly associated with a change in the overall structure of the distal gut microbiome relative to a fish meal diet control, the nature of the change was not consistent in terms of the abundance of individual species targeted by qRT-PCR. The results of this study demonstrate that whole micobiome profiling techniques may be more appropriate for evaluating diet effects on the intestinal microbiome, rather than the targeting of a selection of "indicator species". 


\subsection{Introduction:}

There is an increasing interest in inclusion of plant ingredients to replace fishmeal from aquaculture feeds (Mundheim, et al. 2004; Thiessen, et al. 2004; Heikkinen, et al. 2006; Olsen, et al. 2007; Mansfield, et al. 2010). Soybean, canola and pea are some of the plant ingredients that have been used previously in manufacturing aqua-feeds. Observed effects of feeding these plant-based diets to fish, especially carnivorous fish, include varied levels of inflammation, reduced growth and greater susceptibility to diseases (Krogdahl, et al. 2000; Nordrum, et al. 2000). A potential concern with these conclusions is that they are based on single feeding trials conducted over short periods of time. Variability in experimental parameters from study to study largely prevents direct comparison of results, although there is some evidence from metaanalyses of related studies that some ingredients have consistent effects on growth (S. Collins \& M. Drew, unpublished). However, there are no studies in the literature addressing the reproducibility of the effects of a plant based diet on the structure of the intestinal microbiome. There are few long term studies of the changes taking place in the intestinal microbiota (Holben, et al. 2002; Hagi, et al. 2004), but there is a clear need to evaluate if plant ingredient based diets have a reproducible effects on the structure of intestinal microbiota if the evaluation of the intestinal microbiome is ever going to be useful as a tool for evaluation of diets.

Over the past 30 years the intestinal microbiota of different fish species have been studied using culture based techniques, and the results of these 
studies suggested that the fish intestinal microbiome is not a particularly diverse community, relative to the intestinal microbiomes of terrestrial animals (Cahill. 1990; Ringo, et al. 1995). Recently, culture independent PCR based techniques have been applied to characterise the intestinal microbiota of different fish species, leading to an increased appreciation of the true diversity of this environment (Spanggaard, et al. 2000; Holben, et al. 2002; Huber, et al. 2004; Kim, et al. 2007; Namba, et al. 2007). The advantages of these methods for the characterisation of bacterial communities from soil, water, and aquatic surfaces have been discussed in several recent reviews (Cunliffe, et al. 2011; Erijman, et al. 2011; Maron, et al. 2011).

The cpn60 universal target (UT) has been employed in characterising microbial communities associated with humans and other terrestrial animals (Hill, et al. 2005a; Hill, et al. 2005b; Dumonceaux, et al. 2006a; Desai, et al. 2009), and it has been demonstrated that the cpn60 UT offers greater discriminating power for closely related species compared to the $16 \mathrm{~S}$ rRNA gene (Hill, et al. 2004). Technological advances in pyrosequencing have facilitated rapid characterisation of microbial environments, beyond what is possible with cloning and Sanger sequencing (Sogin, et al. 2006), and these methods have been used in combination with the cpn60 UT to create high resolution profiles of microbial communities (Schellenberg, et al. $2011 \mathrm{a}$; Schellenberg, et al. $2011 b)$.

In a recent study we used cpn60 UT based pyrosequencing to characterise the intestinal microbiome of rainbow trout fed with different plant ingredient 
based diets. The aim of this study was to compare the intestinal microbiome structure associated with plant based diets to that associated with a fish meal control diet (Desai et al. Submitted, Chapter 4). Our results demonstrated that microbiome structures associated with plant ingredients and fish meal were distinguishable and that the difference between them was smaller when the plant ingredients were processed from meal to protein concentrate. Although the results of this work were significant in demonstrating the potential advantages of processing plant ingredients to reduce disturbance of the intestinal microbiome, important questions remain about the reproducibility of the observed plant ingredient effects on the structure of the trout intestinal microbiome, and the extent to which cpn60 amplicon pyrosequencing libraries reflect the actual abundance of bacterial taxa in the intestinal microbiome.

Pyrosequencing of PCR amplicons for microbial community profiling has many potential biases that can affect results interpretation and downstream analysis. Genomic DNA extraction method, PCR conditions, and primer selection can all affect the extent to which the resulting library of sequences reflects the actual composition of the original microbial community sample, both in terms of the species represented and their relative abundance. It has been shown that bacterial sequence frequency can vary over orders of magnitude in a pyrosequencing library even though equal numbers of these bacteria were known to be present in the original sample (Amend, et al. 2010). Despite the wide use of pyrosequencing, studies like this one are rare. If conclusions regarding the biology or ecology of a microbial community or the identification

\section{5}


of diagnostic targets are to be based on pyrosequencing of metagenomic libraries, it is critical to validate the findings with truly quantitative methods such as qRT-PCR.

We predict that the effects of plant ingredient based diets on the intestinal microbiome are reproducible since changes in structure are determined by the nutritional selection imposed on the microbiota by the particular ingredients in each diet. The aim of this study was to identify sequence markers associated with the plant based diets in our original trial (Trial-1) and to compare the abundance of these sequences and the overall intestinal microbiomes of fish from Trial- 1 to those of fish fed with same diets in a second trial (Trial-2). The comparison was based on both the abundance of seven selected bacterial targets in pooled intestinal contents of fish determined by cpn60 based qRT-PCR, and by $16 \mathrm{~S}$ rRNA based DGGE profiling of individual fish. 


\subsection{Material and methods:}

\subsubsection{Fish husbandry}

Trial-1 was conducted at the Prairie Aquaculture Research Centre (University of Saskatchewan, Saskatoon, SK), a biologically filtered semiclosed recirculating aquaculture system. This trial extended over a period of one year and is described in detail in the previous chapter.

Trial- 2 was conducted in the same facility as Trial-1. Triploid female rainbow trout (Oncorhynchus mykiss) were acquired from Wild West Steelhead (Lucky Lake, SK). Fish and diets were randomly assigned to 12 tanks (20 fish / tank; average weight $=270.1 \mathrm{~g}$ ) with three tanks / treatment. Fish were housed in $360 \mathrm{~L}$ tanks with water temperature adjusted at $15 \pm 1{ }^{\circ} \mathrm{C}$ and the photoperiod was $14 \mathrm{~h}$ light: $10 \mathrm{~h}$ dark. Water quality indicators were closely monitored over

the course of the experiment. Fish were acclimatized to the farm environment by housing them for two weeks before trial on a commercial fishmeal based diet. All instructions and guidelines set by the Canadian Council of Animal Care (CCAC, 2005) were followed throughout the experimental trial (University Committee on Animal Care and Supply Protocol 19980142).

\subsubsection{Diets}

All fish reared during Trial-1 and Trial-2 was hand-fed to satiety twice daily with diets formulated using different plant ingredients (soybean, pea and canola) to replace fishmeal. Diet formulation for Trial-1 and Trial-2 are shown 
in Table 5.1 and Table 5.2 respectively. Experimental diets were formulated by replacing $30 \%$ of fishmeal from the control diet with the plant ingredient. All diets were formulated to meet the nutrient requirements of rainbow trout. Two different sources of fishmeal were used during Trial-1 to fulfill the adequate requirements of the trial as indicated in Table 5.1. 
Table 5.1 Ingredient composition of experimental diets.

\begin{tabular}{|c|c|c|c|c|c|c|c|c|}
\hline Ingredient $(\mathrm{g} / \mathrm{kg})$ & $\mathrm{FM}^{*}$ & $\overline{P M}$ & $\mathrm{PPC}$ & $\overline{\text { SBM }}$ & $\mathrm{FM}^{\%}$ & SPC & $\overline{\mathrm{CM}}$ & $\mathrm{CPC}$ \\
\hline Pea meal ${ }^{a}$ & 0.00 & 300.00 & 0.00 & 0.00 & 0.00 & 0.00 & 0.00 & 0.00 \\
\hline $\begin{array}{l}\text { Pea protein concentrate } \\
\text { Soybean } \mathrm{meal}^{\mathrm{b}}\end{array}$ & $\begin{array}{l}0.00 \\
0.00\end{array}$ & $\begin{array}{l}0.00 \\
0.00\end{array}$ & $\begin{array}{l}300.00 \\
0.00\end{array}$ & $\begin{array}{l}0.00 \\
300.00\end{array}$ & $\begin{array}{l}0.00 \\
0.00\end{array}$ & $\begin{array}{l}0.00 \\
0.00\end{array}$ & $\begin{array}{l}0.00 \\
0.00\end{array}$ & $\begin{array}{l}0.00 \\
0.00\end{array}$ \\
\hline Soy protein concentrate ${ }^{d}$ & 0.00 & 0.00 & 0.00 & 0.00 & 0.00 & 300.00 & 0.00 & 0.00 \\
\hline Canola meal ${ }^{\mathrm{e}}$ & 0.00 & 0.00 & 0.00 & 0.00 & 0.00 & 0.00 & 300.00 & 0.00 \\
\hline $\begin{array}{l}\text { Canola } \\
\text { concentrate }\end{array}$ & 0.00 & 0.00 & 0.00 & 0.00 & 0.00 & 0.00 & 0.00 & 300.00 \\
\hline Nova Scotia fish meal ${ }^{g}$ & 390.00 & 387.10 & 275.41 & 299.64 & 0.00 & 0.00 & 0.00 & 0.00 \\
\hline West coast fish meal ${ }^{\mathrm{h}}$ & 0.00 & 0.00 & 0.00 & 0.00 & 400.00 & 217.17 & 452.80 & 407.83 \\
\hline Meat and bone meal ${ }^{1}$ & 224.80 & 0.00 & 56.24 & 45.83 & 304.00 & 89.63 & 0.00 & 0.00 \\
\hline Fish oil ${ }^{\mathrm{J}}$ & 141.34 & 156.31 & 122.03 & 146.54 & 120.50 & 169.86 & 83.60 & 74.28 \\
\hline Alpha-cellulose $\mathrm{k}^{\mathrm{K}}$ & 117.94 & 0.00 & 67.63 & 90.19 & 57.80 & 103.33 & 44.20 & 95.84 \\
\hline Wheat flour ${ }^{1}$ & 100.00 & 47.56 & 100.00 & 100.00 & 100.00 & 100.00 & 100.00 & 100.00 \\
\hline Corn gluten meal & 11.16 & 95.04 & 62.70 & 2.63 & 4.75 & 0.00 & 0.00 & 0.00 \\
\hline Vitamin premix ${ }^{\mathrm{m}}$ & 4.75 & 4.75 & 4.75 & 4.75 & 4.75 & 4.75 & 4.75 & 4.75 \\
\hline Mineral premix ${ }^{n}$ & 4.75 & 4.75 & 4.75 & 4.75 & 4.75 & 4.75 & 4.75 & 4.75 \\
\hline Choline chloride $^{\circ}$ & 4.00 & 4.00 & 4.00 & 4.00 & 4.00 & 4.00 & 4.00 & 4.00 \\
\hline DL-Methionine $\mathrm{e}^{\mathrm{p}}$ & 0.75 & 0.00 & 2.00 & 1.17 & 1.80 & 3.02 & 0.20 & 1.42 \\
\hline$L-L y \sin e^{q}$ & 0.00 & 0.00 & 0.00 & 0.00 & 1.90 & 2.99 & 5.20 & 6.63 \\
\hline Vitamin $\mathrm{C}^{\mathrm{r}}$ & 0.50 & 0.50 & 0.50 & 0.50 & 0.50 & 0.50 & 0.50 & 0.50 \\
\hline
\end{tabular}

${ }^{*}$ FM diet fed as control with PM, PPC and SBM.

${ }^{\%}$ FM diet fed as control with SPC, CM and CPC.

a Yellow field pea, CDC Mozart; Crop Development Centre, Saskatoon, SK, Canada.

${ }^{b}$ Pea protein concentrate, yellow field pea, prestige protein; Parrheim Foods, Saskatoon,

S K, Canada.

${ }^{c}$ Soybean meal; Federated Cooperatives Limited, Saskatoon, SK, Canada.

d Soycomil ${ }^{\circledR}$ K; ADM Special ty Ingredients (Europe) BV, Koog aan de Zaan, The Netherlands.

e Canola Meal-35; Federated Co-Operative Ltd., Saskatoon, SK, Canada.

${ }^{\mathrm{f}}$ Can Pro IP; CanPro Ingredients Ltd., Saskatoon, SK, Canada.

g Nova Scotia herring meal; Shur-Gain Aquaculture, Truro, NS, Canada.

h West Coast Fish Meal; Federated Co-Operative Ltd., Saskatoon, SK, Canada.

${ }^{i}$ Saskatoon Processing Co.; Saskatoon Processing Co., Saskatoon, SK. Canada.

j Danish Fish Oil; FF of Denmark, Skagen, Denmark.

${ }^{k}$ Solka-floc ${ }^{\circledR}, 200$ FCC; International fiber corporation, North Tonawanda, NY, USA.

l Robin Hood All-Purpose Flour; Robin Hood Multifoods Corporation, Markham, ON, Canada.

${ }^{m}$ DL-me thi oni ne, feed grade. Degussa Corporation, Theodore, AL, USA.

n Vitamin premix, commercial (EWOS FISH-STR VIT PX, Surrey, BC; closed formulation), formulated to meet the requirements of juvenile rainbow trout; BASF Canada, Surrey, BC, Canada.

${ }^{0} 60 \%$ Choline Chloride; Chinook Group Limited Partnership, Sombra, ON, Canada.

p DL-methionine, feed grade. Degussa Corporation, Theodore, AL, US A.

${ }^{q}$ L-Lysine, monohydrochloride; Ajinomoto Heartland LLC, Eddyville, IA, USA.

${ }^{\mathrm{r}}$ Ascorbic acid, pharmaceutical grade; NOW Foods, Bloomingdale, IL, USA. 
Table 5.2 Ingredient composition of experimental diets for Trial-2.

\begin{tabular}{|c|c|c|c|c|}
\hline Ingredient $(\mathrm{g} / \mathrm{kg})$ & $0 \mathrm{~g} / \mathrm{kg}$ & $260 \mathrm{~g} / \mathrm{kg} \mathrm{PM}$ & $260 \mathrm{~g} / \mathrm{kg} \mathrm{SBM}$ & $260 \mathrm{~g} / \mathrm{kg} \mathrm{CM}$ \\
\hline Pea meal ${ }^{\mathrm{a}}$ & 0.00 & 260.00 & 0.00 & 0.00 \\
\hline Soybean meal ${ }^{\mathrm{b}}$ & 0.00 & 0.00 & 260.00 & 0.00 \\
\hline Canola meal ${ }^{\mathrm{c}}$ & 0.00 & 0.00 & 0.00 & 260.00 \\
\hline Fish meal ${ }^{d}$ & 614.14 & 466.39 & 395.91 & 458.27 \\
\hline Diatomaceous earth ${ }^{\mathrm{e}}$ & 180.86 & 0.00 & 119.19 & 93.80 \\
\hline Fish oil ${ }^{\mathrm{f}}$ & 119.55 & 144.66 & 136.46 & 101.82 \\
\hline Wheat flour ${ }^{\mathrm{g}}$ & 47.00 & 47.00 & 47.00 & 47.00 \\
\hline Porcine blood plasmah & 20.00 & 63.12 & 20.00 & 20.00 \\
\hline Vitamin premi $x^{i}$ & 4.75 & 4.75 & 4.75 & 4.75 \\
\hline Mineral premi ${ }^{j}$ & 4.75 & 4.75 & 4.75 & 4.75 \\
\hline Choline chloride $\mathrm{e}^{\mathrm{k}}$ & 4.00 & 4.00 & 4.00 & 4.00 \\
\hline L-ly s in $\mathrm{e}^{1}$ & 3.23 & 2.13 & 4.07 & 4.51 \\
\hline L-Methionine ${ }^{\mathrm{m}}$ & 1.22 & 2.71 & 2.36 & 0.61 \\
\hline Vitamin $C^{\mathrm{n}}$ & 0.50 & 0.50 & 0.50 & 0.50 \\
\hline
\end{tabular}

\footnotetext{
${ }^{a}$ Yellow field pea, CDC Mozart; Crop Development Centre, Saskatoon, SK, Canada.

${ }^{b}$ Soybean meal; Federated Cooperatives Limited, Saskatoon, SK, Canada.

e Canola Meal-35; Federated Co-Operative Ltd., Saskatoon, SK, Canada.

${ }^{d}$ West Coast Fish Meal; Federated Co-Operative Ltd., Saskatoon, SK, Canada.

e Celite 545, <125 $\mu \mathrm{m}$; Celite Corporation, World Minerals Co., Lompoc, CA, USA.

${ }^{\mathrm{f}}$ Mixed Species Fish Oil; Bioriginal Food and Science Corp., Saskatoon, SK, Canada.

g Robin Hood All-Purpose Flour; Robin Hood Multifoods Corporation, Markham, ON, Canada.

${ }^{\text {h }}$ Saskatoon Processing Co.; Saskatoon Processing Co., Saskatoon, SK. Canada.

i Vitamin premix, commercial (EWOS fish feed vitamin premix; closed formulation), formulated to meet the requirements of juvenile rainbow trout; BASF Canada, Surrey, BC, Canada.

j Mineral premix, commercial (EWOS fish feed mineral premix; closed formulation), formulated to meet the requirements of juvenile rainbow trout; BASF Canada, Surrey, BC, Canada.

${ }^{k} 60 \%$ Choline Chloride; Chinook Group Limited Partnership, Sombra, ON, Canada.

${ }^{1}$ L-lysine HCL $788 \mathrm{~g} / \mathrm{kg}$; Archer Daniels Midland Company, Decatur, IL, USA.

${ }^{m}$ DL-me thi onine, feed grade. Degussa Corporation, Theodore, AL, USA.

${ }^{\mathrm{n}}$ Ascorbic acid, pharmaceutical grade; NOW Foods, Bloomingdale, IL, USA.
} 


\subsubsection{Sample collection and DNA extraction}

At the end of the feeding period of 56 days for Trial-1 and 39 days for Trial-2, nine fish from the experimental diet group and nine fish from the control group were selected randomly and euthanized (three fish from each of three tanks for each diet group). Distal gut contents were collected as described earlier (Mansfield, et al. 2010). Briefly, the gastrointestinal tract was dissected and the distal gut contents were squeezed out into a sterile tube and held on ice prior to storage at $-80{ }^{\circ} \mathrm{C}$ until DNA extraction. Total genomic DNA was extracted from the distal gut contents of individual fish using the QIAamp ${ }^{\circledR}$ DNA Stool Mini Kit (Qiagen Inc., Mississauga, ON).

\subsubsection{Quantitative real-time PCR}

Targets for qRT-PCR were selected based on the basis of an observed significant difference in the proportional sequence abundance between the experimental diet fish or their corresponding FM-control fish. Bacterial sequence frequencies were combined based on their nearest neighbour in cpnDB to form operational taxonomic units (OTU). Significant differences in OTU abundance where detected using Levene's test to determine the equality of variance followed by Mann-Whitney $U$ test (for unequal variance) using SPSS software (SPSS Inc. Chicago, IL, USA). Cut-off for statistical significance was $p<0.05$. Specific qRT-PCR primers based on cpn60 UT sequences for seven bacterial targets were designed using Primer3Plus software (Untergasser, et al. 2007).

\section{1}


Specificity of the primers was evaluated initially by comparing the primer sequences to the Genbank sequence database and cpnDB (Hill, et al. 2004). Primer sequences, annealing temperatures, product sizes are shown along with target descriptions in Table 5.3. Generation of PCR product for cloning to create positive control plasmids was performed on pooled genomic DNA from all fish in a diet group from Trial-1 where the target of interest was detected in relative abundance by pyrosequencing (i.e. a template pool most likely to yield product for cloning). PCR products were amplified using primers specific for each target as shown in Table 5.3. PCR reactions consisted of $1 \times$ PCR reaction buffer (20mM Tris- $\mathrm{HCl}(\mathrm{pH} 8.4), 50 \mathrm{mM} \mathrm{KCl}), 2.5 \mathrm{mM} \mathrm{MgCl} \mathrm{m}_{2}, 200 \mu \mathrm{M}$ dNTPs, $400 \mathrm{nM}$ of forward and reverse primer, 2.5 U Platinum Taq DNA polymerase (Invitrogen, Burlington, ON, Canada) and $2 \mu 1$ template DNA, in a final volume of $50 \mu 1$. Twelve PCR reactions were run for each target using a BioRad MyiQ thermocycler. Cycling parameters were set at $94{ }^{\circ} \mathrm{C}$ for $3 \mathrm{~min}$, followed by 40 cycles of $30 \mathrm{sec}$ at $94{ }^{\circ} \mathrm{C}, 1 \mathrm{~min} 55-67{ }^{\circ} \mathrm{C}$ annealing gradient and $30 \mathrm{sec}$ at $72{ }^{\circ} \mathrm{C}$, followed by final extension for $10 \mathrm{~min}$. PCR products were pooled together and gel purified. PCR products were ligated into cloning vector pGEM T Easy (Promega Co., Madison, WI) and inserts were sequenced for confirmation of the correct insert. Optimal annealing temperatures for all the qRT-PCR assays were determined by running an annealing temperature gradient PCR on the generated plasmid standards representing each target. All PCR were performed on MyiQ thermocycler (BioRad) as described above. All the targets were subjected to a dissociation curve analysis after the PCR run. Quantification

\section{2}


of the targets in individual pooled samples was accomplished through the use of a standard curve derived from a ten fold dilution series of plasmid DNA containing cpn60 sequence from each target $\left(10^{1}-10^{8}\right.$ copies per reaction)(Dumonceaux, et al. 2006b) 


\section{Table 5.3 qRT-PCR assays for quantification of bacteria.}

\begin{tabular}{|c|c|c|c|c|}
\hline Target & Nearest neighbour in cpnDB & Primer sequence $\left(5^{\prime}-3^{\prime}\right)$ & Annealing temp $\left({ }^{\circ} \mathrm{C}\right)$ & Amplicon size (bp) \\
\hline FIR-1 & Hafnia alvae & $\begin{array}{l}\text { GT T CGAT CGT GGCT ACCT GT } \\
\text { AGT GGT T T GCCT GCT T T AGCA }\end{array}$ & 67 & 158 \\
\hline FIR-2 & Weissella paramesenteroides & $\begin{array}{l}\text { GT GT GCGT CGT GGT AT CGAAT T } \\
\text { CAACT CACCAACT T CT GGGT T A }\end{array}$ & 64 & 137 \\
\hline FIR-3 & Lactococcus garvieae & $\begin{array}{l}\text { AAAGAGT T GT CT AT T CCT GT AT } \\
\text { AT T GCAT ACCT T CCACAACT TC }\end{array}$ & 61 & 187 \\
\hline PR0 - 1 & Raoultella terrigena & $\begin{array}{l}\text { GT T GCT GCT GT T GAAGAGCT } \\
\text { T CACGCCT T CT T TACCGACT }\end{array}$ & 67 & 153 \\
\hline PRO-2 & Acidovorax facilis & $\begin{array}{l}\text { CCAACT CT GACGA AACCAT CGG } \\
\text { AAGT A GGGCGACA GGT A GCC }\end{array}$ & 67 & 156 \\
\hline PRO-3 & Shewanella oneidensis & $\begin{array}{l}\text { AGAAACT GGCAGCGT T GAAT } \\
\text { T AAT GGCT T ACCGGT T T T A GCC }\end{array}$ & 55 & 120 \\
\hline PRO-4 & Enterobacter amnigenus & $\begin{array}{l}\text { AAGGT CT GAAA GCGGT CGCT GC } \\
\text { CGGT ACGGACA GGGT T T T CAGC }\end{array}$ & 67 & 120 \\
\hline
\end{tabular}


Equal volumes of genomic DNA extracts from the intestinal contents of fish fed with the same diet were pooled to form one template DNA pool, resulting in twelve diet group templates from Trial-1 (PM, FM $\mathrm{PM}_{\mathrm{P}}$, PPC, FM $\mathrm{MPC}_{\mathrm{PPC}}$ $\mathrm{SBM}, \mathrm{FM}_{\mathrm{SBM}}, \mathrm{SPC}, \mathrm{FM}_{\mathrm{SPC}}, \mathrm{CM}, \mathrm{FM}_{\mathrm{CM}}, \mathrm{CPC}, \mathrm{FM}_{\mathrm{CPC}}$ ), and four template pools from Trial-2, three representing experimental diets and one representing the FM-control group.

Quantitative PCR reactions for detection of specific targets included $1 \times$ SYBR Green Supermix (Biorad Laboratories, Mississauga, ON), $400 \mathrm{nM}$ of each primer (Table 5.3), and $2 \mu 1$ of template DNA extract in a final volume of $25 \mu 1$. Cycling parameters were set at $95{ }^{\circ} \mathrm{C}$ for $3 \mathrm{~min}$, followed by 40 cycles of $10 \mathrm{sec}$ at $95{ }^{\circ} \mathrm{C}, 30 \mathrm{sec}$ at the appropriate annealing temperature (Table 5.3), and $30 \mathrm{sec}$ at $72{ }^{\circ} \mathrm{C}$, followed by a dissociation curve stepping from $65{ }^{\circ} \mathrm{C}$ to $95{ }^{\circ} \mathrm{C}$ in $0.5{ }^{\circ} \mathrm{C}$ increments. Reactions were run in duplicate on a Biorad MyiQ thermocycler (Biorad Laboratories, Mississauga, ON). Negative (no template) controls were included with each assay. Determination of target copy number was based on a standard curve generated from a tenfold dilution series of the appropriate target sequence plasmid. In cases where both duplicates gave a consistent $\mathrm{C}_{t}$ greater than the lowest standard and product was confirmed by appearance of the appropriate peak in the dissociation curve, the sample was declared to be detectable, but not quantifiable (DNQ).

\section{5}




\subsubsection{S rRNA PCR-DGGE and cluster analysis}

PCR was performed with universal primers F-968-GC and R-1401 flanking the V6-V8 region of the 16S rRNA gene (Nubel, et al. 1996) as described previously (Konstantinov, et al. 2003). PCR amplicon separation was done on an $8 \%$ polyacrylamide gel with a gradient of $45-60 \%$ urea as described previously (Janczyk, et al. 2007). Marker lanes containing size standards were run in the outermost lanes of each gel. DNA size standards were composed of $16 \mathrm{~S}$ rRNA V6-V8 PCR products of bacterial isolates and clones from the distal gut of rainbow trout. Gels were stained with SYBR Gold Nucleic Acid Gel Stain (Invitrogen Canada, Burlington, ON) and visualized and photographed under ultraviolet light.

DGGE fingerprints were normalized and analyzed using GelCompar II V6.1 software (Applied Maths Inc., Austin, TX). Percent similarity indices of the fingerprints were calculated using the Pearson product-moment correlation. Dendrograms of the fingerprints were built using the calculated Pearson correlation un-weighted pair group method algorithm (UPGMA). 


\subsection{Results:}

\subsubsection{Validating pyrosequencing findings using $q R T-P C R$ :}

Seven target specific qRT-PCR assays were applied to distal gut DNA extract pools from Trial-1 (12 template DNA pools) to validate the findings from pyrosequencing. All targets were selected on the basis of their abundances in experimental diet and their corresponding controls. Consequently we compared the trend of copy number for individual targets from Trial-1 and Trial-2 (Figures 5.1 and 5.2). The detection limit for all qRT-PCR assays was determined to be $5 \times 10^{2}$ target copies $\mathrm{g}^{-1}$ of intestinal contents. Target copy numbers detected in Trial-1 samples ranged from undetectable to $2 \times 10^{9}$ copies $\mathrm{g}^{-1}$.

Comparison of the pyrosequencing frequency data and qRT-PCR copy numbers for FIR-1, FIR-2 and FIR-3 (Figure 5.1) showed that results from both techniques did not always agree. Frequency data generated using pyrosequencing showed that FIR-1 sequences were significantly more abundant in $\mathrm{CM}$ as compared to its FM control diet (Figure 5.1A). A similar trend was seen when copy numbers for the se targets were generated for CM associated fish. A significant difference for FIR-1 frequency was also seen in PPC and its corresponding control but similar trend was not seen by qRT-PCR as the target was detected but not quantifiable (Figure 5.1A). On the other hand both FIR-1 $\left(4.46 \times 10^{7}\right.$ target copies $\left.\mathrm{g}^{-1}\right)$ and FIR-3 (4.53 $\times 10^{6}$ target copies $\left.\mathrm{g}^{-1}\right)$ were detected in PM associated fish by qRT-PCR but the frequency numbers by 
pyrosequencing did not show a similar trend. Target FIR-2 was one of the most inconsistent targets. It was detectable but not quantifiable in CM, CPC and their corresponding FM control associated fish by qRT-PCR but sequences corresponding to this target were detected in abundance in the corresponding pyrosequencing libraries (Figure 5.1B). FIR-2 sequence frequency and qRT-PCR data (more than $10^{6}$ target copies $\mathrm{g}^{-1}$ ) for fish associated with PM, PPC and SBM were in agreement, where the frequency of FIR-2 was always higher than 1600 reads (Table 5.4). However, while FIR-2 sequence frequency in fish associated with the corresponding control diets was also higher than 1000, it was detected at very low levels (DNQ) by qRT-PCR. 


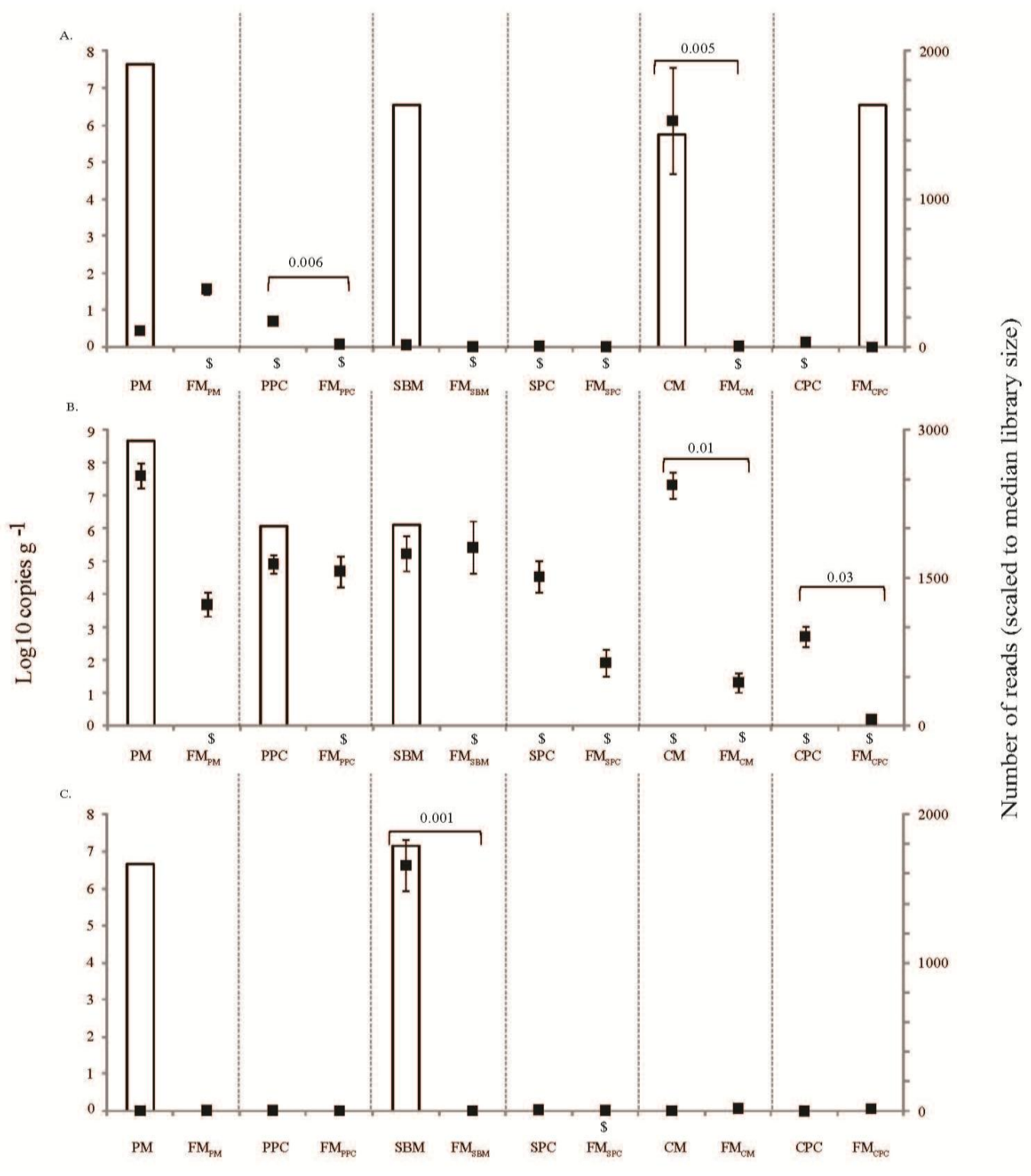

Figure 5.1 Comparison of qRT-PCR copy numbers (bars, left ordinate) and target sequence frequency (squares, right ordinate) of (A) FIR-1, (B) FIR-2 and (C) FIR-3 from Trial-1. Sequence abundance data is plotted as the mean of the scaled sequence abundance of all fish in each diet group $(n=9$, except for PPC,CM, FM $\left.\mathrm{MPC}_{\mathrm{SPC}}, \mathrm{FM}_{\mathrm{CM}}, \mathrm{FM}_{\mathrm{CPC}}\right)$. Error bars indicate standard deviation. Significant differences $(\mathrm{p}<0.05)$ in sequence abundance between experimental and control diet pairs are indicated by $p$ value. qRT-PCR copy numbers were generated for pooled genomic DNA from intestinal contents of all fish in each diet group. \$ represents detected but not quantifiable.

Results for targets PRO-1, PRO-2, PRO-3 and PRO-4 were also inconsistent (Figure 5.2). Sequences corresponding to targets PRO-1, PRO-2 and 
PRO-4 were significantly more abundant in fish fed with PM than the corresponding FM control and this trend was also seen by qRT-PCR (Figure 5.2 A, B and D). In the other diets, PRO-1 (Figure 5.2A) results were consistent in that the sequence frequencies for all the diets were fewer than 32 and the target was either undetected or detectable but not quantifiable by qRT-PCR. PRO-2 (Figure 5.2B) sequences were significantly more abundant in all experimental diet fish compared to their control diet counterparts, but it was detected by qRT-PCR at quantifiable levels only in only fish fed the PM diet (2 X $10^{7}$ target copies $\mathrm{g}^{-1}$ ). PRO-3 (Figure 5.2C) showed mixed results where findings by both methods agreed in the cases of CM and SBM fish but did not agree in the other diet groups. PRO-3 was detected by qRT-PCR at a range from undetectable to $2 \times 10^{8}$ target copies $\mathrm{g}^{-1}$. PRO-4 (Figure 5.2D) showed similar trends by both qRT-PCR and pyrosequencing in PM and PPC fish, where a significant difference was seen in sequence frequency between experimental diets and controls. For SPC fish a significant difference in frequency was seen

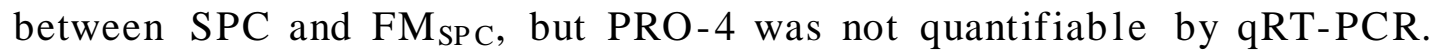




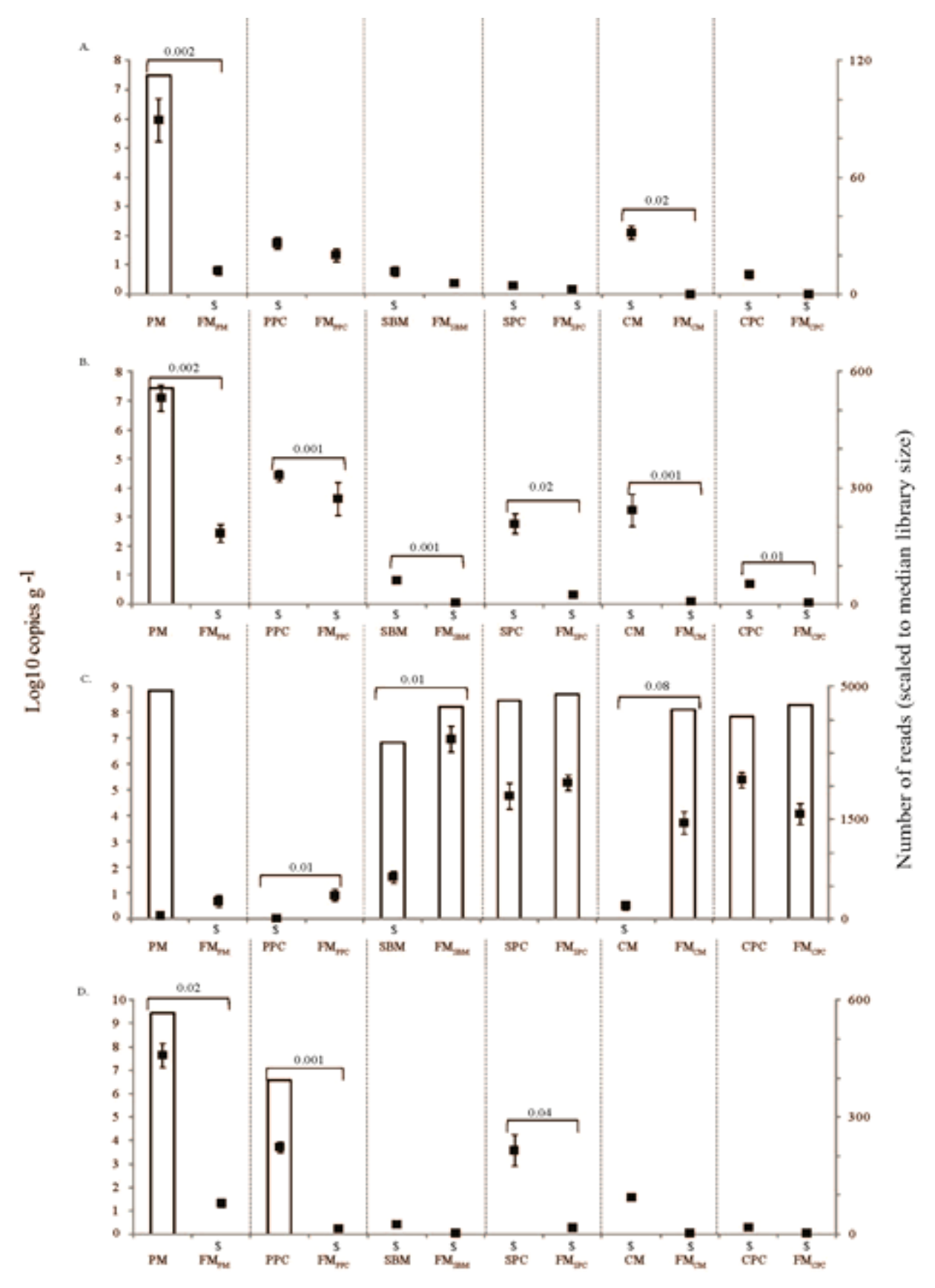

Figure 5.2 Comparison of qRT-PCR copy numbers (bars, left ordinate) and target sequence frequency (squares, right ordinate) of (A) PRO-1, (B) PRO-2 (C) FIR 3 and (D) PRO-4 from Trial-1. Sequence abundance data is plotted as the mean of all fish in each diet group ( $\mathrm{n}=9$, except for PPC,CM, FM $\mathrm{SPC}_{\mathrm{SP}}, \mathrm{FM}_{\mathrm{CM}}$, $\left.F_{C P C}\right)$. Error bars indicate standard deviation. Significant differences $(p<0.05)$ in sequence abundance between experimental and control diet pairs are indicated by $\mathrm{p}$ value. qRT-PCR copy numbers were generated for pooled genomic DNA from intestinal contents of all fish in each diet group. \$ represents detected but not quantifiable. 


\subsection{2 qRT-PCR based comparison of target abundance in Trial-1 and Trial-2.}

A second goal of the study was to determine if diet associated changes in microbiome structure were reproducible by comparing diet associated shifts in abundance of bacterial species targets in fish from Trial-1 and Trial-2 using qRT-PCR on pooled samples from each diet group. Comparison of copy numbers of targets in Trial-1 and Trial-2 were generally inconsistent. For example FIR-1 was detected in PM fish from both trials at approximately $10^{7}$ target copies $\mathrm{g}^{-1}$, but was only detected at quantifiable levels in FM control fish from Trial-2 at $1.12 \times 10^{7}$ target copies $\mathrm{g}^{-1}$ (Figure 5.3A). This inconsistent trend was true in case of all the targets (Figure 5.3). When targets were detected in fish fed experimental diets in Trial- 2 they were also detected in fish fed experimental diets in Trial-1 (except for FIR-3 and PRO-3 in case of CM), while on the other hand when the target was detected in Trial-1 fish it was not always detected in fish fed with experimental diets during Trial-2. Targets PRO-1 and PRO-2 were consistently undetected or were detected at levels below the quantification limit in the CM and SBM groups. Similarly, FIR-3 was not detected in any SBM fish or their corresponding controls. 

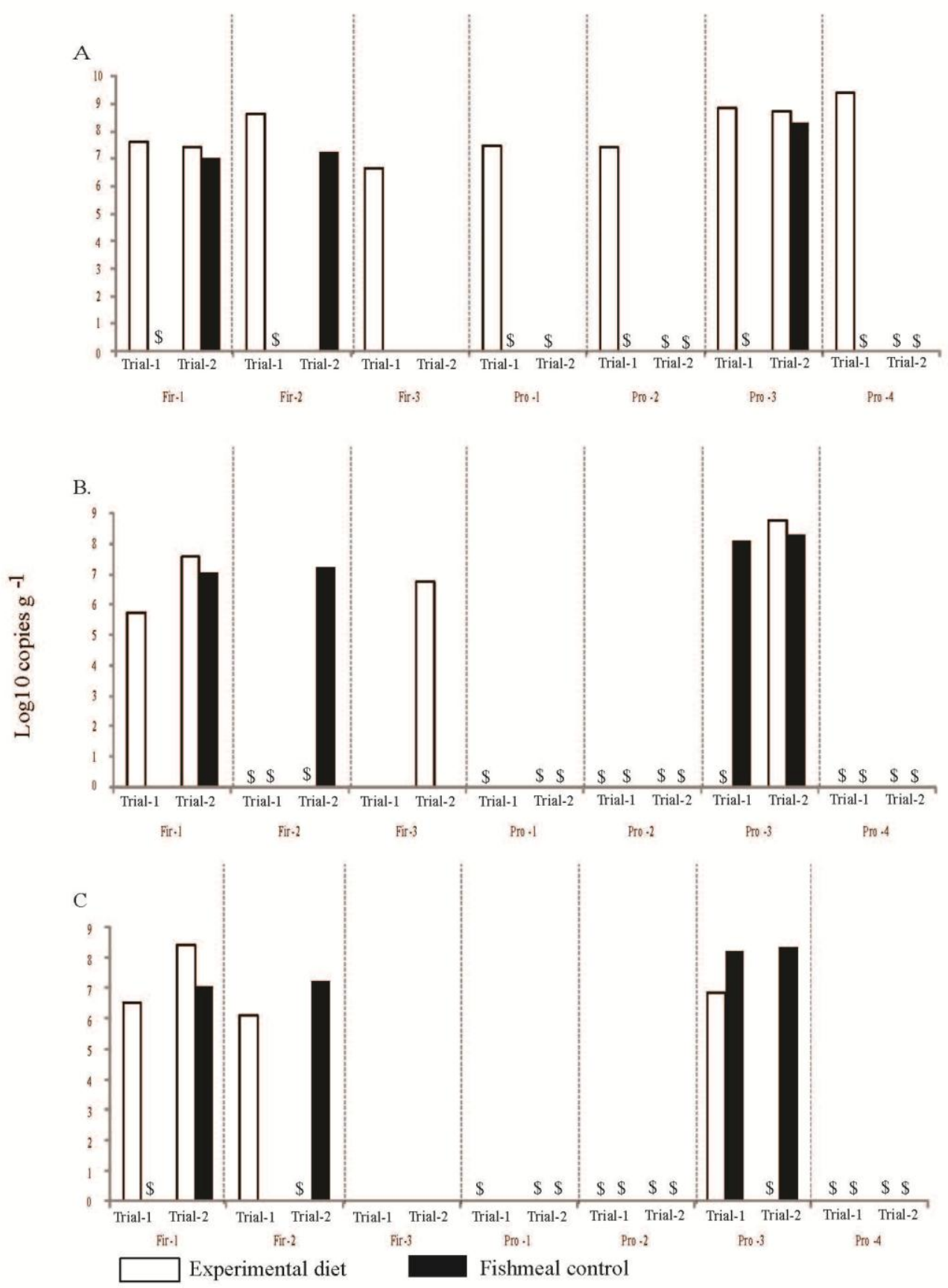

Figure 5.3 Comparison of the copy numbers of the seven bacterial targets in pooled intestinal contents of fish fed with experimental diets (A) PM, (B) CM and (C) SBM in Trial-1 and Trial-2, determined by qRT-PCR. \$ represents detected but not quantifiable. 
5.4.3 Evaluating effects of plant ingredient based diets on intestinal microbiota using $16 S$ rRNA based DGGE:

DGGE intestinal microbial profiles of fish fed with plant based ingredients in Trial-1 were compared to DGGE profiles of fish fed with same diets in Trial-2. DGGE analys is was performed on intestinal contents of individual fish fed with four diets from Trial-2 $\left(\mathrm{FM}_{\mathrm{T} 2}, \mathrm{PM}_{\mathrm{T} 2}, \mathrm{CM}_{\mathrm{T} 2}\right.$ and $\left.\mathrm{SBM}_{\mathrm{T} 2}\right)$ and compared to DGGE profiles generated in Trial-1 (PM, CM and SBM, and their corresponding $\mathrm{FM}$ control diets $\mathrm{PM}_{\mathrm{FM}}, \mathrm{CM}_{\mathrm{FM}}$ and $\mathrm{SBM}_{\mathrm{FM}}$ ) (Chapter 4).

Results based on cluster analysis of DGGE profiles from fish fed the experimental and control diets during Trial- 2 showed that the experimental diet fish always clustered separately from fish fed with fishmeal control diets (Figure 5.4). Minimum percent similarities between experimental and control diet DGGE profiles were $<60 \%$ for all groups.

Comparison of DGGE profiles of fish from Trial-1 and Trial-2 showed that although DGGE profiles of fish fed with plant ingredients always clustered separately from those fed the FM control diet, there was consistently separate clustering of fish reared during Trial-1 and Trial- 2 (Figure 5.5). 

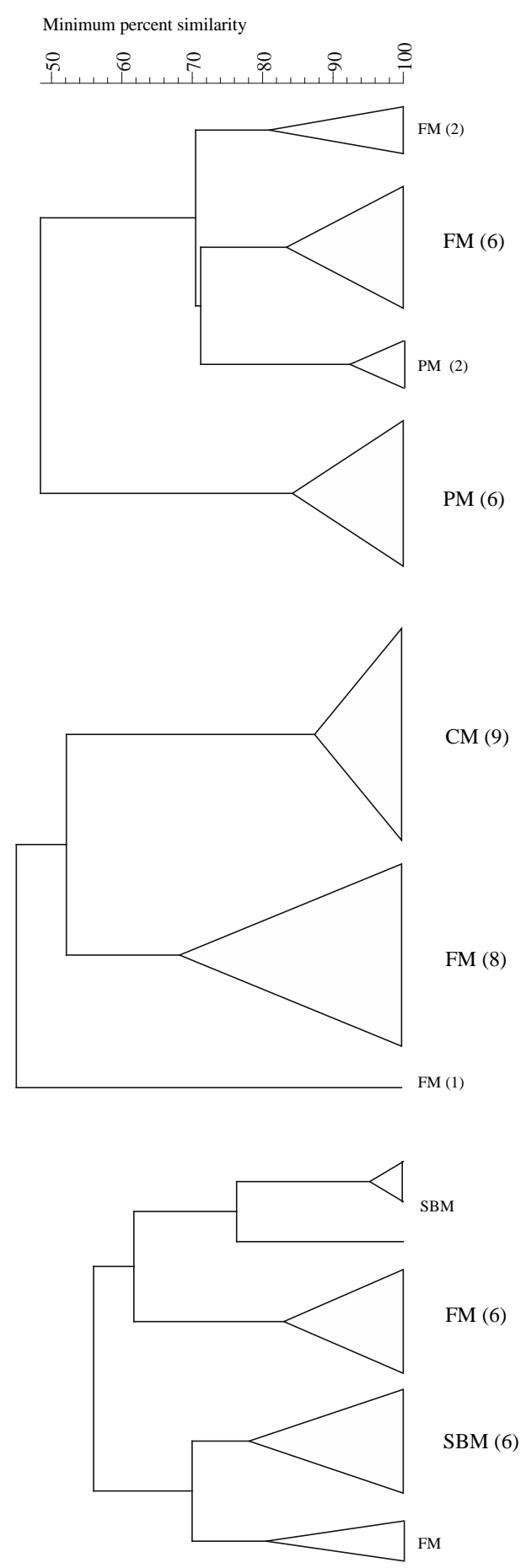

Figure 5.4 Cluster analysis of 16S rRNA DGGE profiles from individual fish fed with PM (top), CM (middle) or SBM (bottom) during Trial-2. 

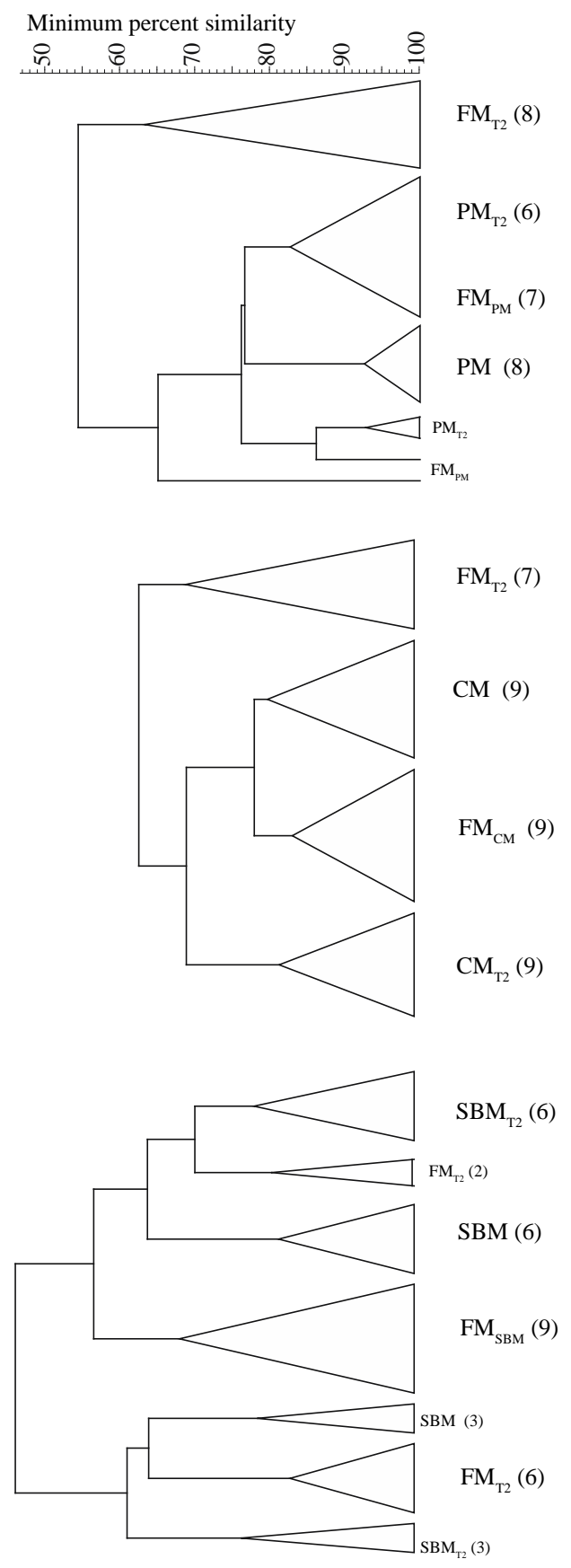

Figure 5.5 Clustering of 16S rRNA DGGE profiles of individual rainbow trout fed PM (top), CM (middle) or SBM (bottom) in Trial-1 and Trial-2. Trees are collapsed at the lowest common node for each diet group. Numbers in parentheses indicate number of DGGE fingerprints assessed. 


\subsection{Discussion:}

Pyrosequencing is a fast and cost effective technique compared to traditional Sanger sequencing, which has enabled high throughput sequencing of different microbial communities (Hugenholtz, et al. 2008). Pyrosequencing allows sequencing of multiple samples in parallel, facilitating comparison of sequence libraries from different samples. Even though pyrosequencing is a useful technique for characterisation of microbial communities, it necessarily results in proportional data, which does not provide absolute quantitative information. Furthermore, since the detection of any sequence in a library of DNA templates is dependent on the composition of that sample, the representation of any individual microbial community member is not assured, even if it is abundant. Unfortunately, since the advent of metagenomic pyrosequencing there has been little effort to validate the results of these sequencing studies using truly quantitative techniques. qRT-PCR is a highly sensitive and specific state of the art technique which has been used to quantify different bacterial targets within bacterial communities (Suzuki, et al. 2000; Yu, et al. 2005; Desai, et al. 2009; Mansfield, et al. 2010). The current study was designed to apply qRT-PCR to validate cpn60 based metagenomic sequence libraries from the intestinal microbiomes of fish fed with different plant based diets, and to apply the species-specific qRT-PCR tools to determine if the changes in intestinal microbiome structure associated with plant ingredient based diets are reproducible. 
In our previous study rainbow trout were fed with different experimental diets in which pea, soy bean and canola were used to replace up to $30 \%$ of fishmeal. Pyrosequencing of cpn60 amplicons was used to define the structure of the intestinal microbiome and describe differences in the intestinal microbiota of these fish fed with different diets. To validate our findings from the pyrosequencing study, seven target specific qRT-PCR assays were developed. These targets were chosen from a group of 22 OTU found to differ significantly in abundance between fish fed with experimental diets or the FM control diet (Table 5.1 and 5.2). Comparing OTU sequence abundance and copy numbers from qRT-PCR analysis showed that neither the absolute numbers nor the trends agreed in all the cases. In some cases, such as FIR-1 abundance in CM and FIR3 abundance in SBM fish, pyrosequencing and qRT-PCR results were in agreement both, although this trend was not consistent in all case of all targets.

Errors such as the misinterpretation of homopolymeric sequence regions are inherent to the 454 pyrosequencing technology and can result in artefactual diversity (Quince, et al. 2009). Other sources of artefact include Taq DNA polymerase infidelity during amplification (Eckert, et al. 1991). Accumulation of these artefacts can result in an overestimation of sequence diversity. On the other hand we have seen earlier that there can be a species and a strain level diversity in any microbial environment (Mansfield, et al. 2010; Vermette, et al. 2010). To simplify the design of qRT-PCR assays and account for some level of diversity overestimation, we chose to create OTUs by pooling the assembled sequences (isotigs) according to their nearest neighbour in the cpnDB reference

\section{8}


database. As shown in Table 5.4, some of these OTU included a large number of is otig sequences (up to 101 for PRO-3). In most cases, the combined sequences were very similar to each other, showing a narrow range of sequence identity values to their nearest neighbour. However, in other cases, such as FIR-2, FIR3 and PRO-3, there was more intra-OTU diversity, raising the possibility that the qRT-PCR primers for that target actually amplify a relatively small proportion of the sequences considered in the sequence abundance comparison. To minimize this effect, qRT-PCR assays developed in these studies were designed in such a way that they detected most of the isotig sequences that were pooled into formation of individual OTU, with primers designed to anneal to conserved sequences within the OTU, avoiding variable regions.

The inconsistency seen while comparing the findings in two techniques may also be due to the fact that the techniques are designed to answer different questions. qRT-PCR is used to target and quantify specific targets which are part of a microbial community, independently of the other community members. Metagenomic libraries are created to characterise the whole microbial community by exploiting "universal" PCR primers, and thus the detection of individual members of the community are not independent. Rare species in the community may be over-represented or abundant species under-represented depending upon the efficiency of their amplication in the universal primer PCR. For example, bifidobacteria and mollicutes are routinely under-represented in 16S rRNA based metagenomic libraries (Hill, et al. 2010; Hummelen, et al. 2011).

\section{9}


Another important factor in our experimental design that may have contributed to the lack of agreement between metagenomic libraries and qRTPCR results is that the cpn60 metagenomic libraries were created from intestinal contents of individual fish, whereas qRT-PCR was performed on pooled genomic DNA from all fish on from each diet group. Inconsistent trends seen in the comparison of the two techniques can be a result of this pooling effect. It has been shown earlier that although it is convenient and economical, pooling samples can result into over or under representation of targets in the community (Desai, et al. 2009).

The observation that plant ingredients result in an intestinal microbiome distinct in structure from that of fish fed the FM control diet was confirmed by DGGE fingerprinting of individual fish from Trial-2 (Figure 5.4). This observation is in agreement with the results we have seen earlier (Chapter 4) where the intestinal microbiota of fish fed with the experimental diet was always distinguishable from the FM control group.

The qRT-PCR assays developed for validation of the metagenomic libraries were also applied to samples from fish in Trial-1 and Trial-2 to determine if differences in abundance of individual species targeted between the control and experimental diets were reproducible. Examination of the data summarized in Figure 5.3 shows that out of 21 cases ( 7 targets for three ingredients), the same trend in relative abundance (increase or decrease regardless of absolute value) between experimental diet and FM control was seen in Trial-1 and Trial-2 in 11 cases. However, when absolute abundance is

\section{0}


considered, the results are even less consistent, with the only true replicates being cases where overall abundance is in the DNQ range (for example, PRO-2 in $\mathrm{CM}$ and $\mathrm{SBM}$ ).

The best explanation for our observation that the apparent abundance of the qRT-PCR targets in the trials were different (Figure 5.3) is found in the combined results of $16 \mathrm{~S}$ rRNA DGGE analys is from the two tria ls (Figure 5.5). The DGGE profiles clustered by trial first, and then by diet within each trial, suggesting that the intestinal microbiomes of the two cohorts of fish were different from each other even before the feeding trials began. Even if the selective pressures from the diet composition were equivalent in the two trials, the effects on the overall structure of the microbiome would be influenced by the composition of the starting population upon which the selection acts. The developmental stage of fish can also determine the structure of the intestinal microbiota (Holben, et al. 2002; Hagi, et al. 2004). Since the fish used in Trial1 and Trial-2 were from different batches and we did not control for the difference in the age, the findings in the study may also have been affected by these factors.

Part of the motivation for this study was to determine if we could identify "indicator species" that could be targeted as a proxy for overall changes in the intestinal microbiome, with the goal that such targets would be diagnostically informative in evaluating the effects of diet and ingredients on the intestinal microbiome. Measuring the abundance of a few selected targets in pooled samples would be simpler and less expensive than generating detailed microbial

\section{1}


sequence profiles for samples from individual fish. However, our results suggest that a species-specific approach may be too specific, and not allow for strain variation within species. Also, the success of this approach assumes that the composition of the trout intestinal microbiome is composed of a consistent repetoire of species, which our results do not support.

The goal of evaluating the intestinal microbiome of aquaculture fish fed different diets is to identify plant ingredient based diet formulations that result in an intestinal microbiome similar in structure to that observed in healthy fish with ideal growth characteristics, which for carnivorous fish would be the microbiome resulting from a diet based on fish meal. Our results suggest that this might best be evalutated by comparing overall population structure using a profiling technique such as DGGE or metagenomic sequencing and looking for a minimal shift away from the FM profile. Rapidly evolving technology and decreasing costs will make sequencing technologies even more accessible in the future. Alternatively, further study may reveal other informative patterns such as ratios of higher taxonomic levels (e.g. Firmicutes:Proteobacteria ratio), microbial gene expression patterns or functional characteristics of the microbiome that are more diagnostically informative than phylogenetic profiling based on targets such as $16 \mathrm{~S}$ rRNA or cpn60. 
Chapter 6 Discrimination of Carnobacterium maltaromaticum strains isolated from rainbow trout (Onchorhynchus mykiss) intestinal contents fed with different plant based diets. 


\subsection{Abstract:}

Many studies have shown that diet plays an important role in the structure of the intestinal microbiota. In addition to diet there are other factors which may affect the intestinal microbiota such as fish age, and environmental factors such as water temperature and salinity. As part of an ongoing series of feeding trials to explore the effects of diet on the microbiome, fish were reared in an indoor recirculating facility. The desgin of the facility results in the fish sharing a common environment that might facilitate the sharing of a common population of intestinal bacteria among fish, reducing the degree of any diet effects observed. We have used Carnobacterium maltaromaticum as a target species to determine the extent to which different strains of $C$. maltaromaticum were shared among fish in the same environment, regardless of their diet. $C$. maltaromaticum is known to be part of the intestinal microbiota of rainbow trout (Onchorhynchus mykiss) and is recognized as both a beneficial and a pathogen. Here we have used $\mathrm{GTG}_{5}$ rep PCR to discriminate between $C$. maltaromaticum strains isolated from the intestinal microbiota of rainbow trout fed with different plant based diets. Results from this study suggest that the intestinal microbial profile of individual fish is unique at the strain level. Findings from this study will help us in understanding effects of shared environments on the intestinal microbiota of rainbow trout.

\section{4}




\subsection{Introduction:}

The intestinal microbiota of fish is influenced by many factors such as seasons, environment variations, age, and diet (Ringo, et al. 1995; Hovda, et al. 2011). Previous characterisations of intestinal microbiota relied heavily on culture based techniques hence limiting our knowledge to bacterial species which are readily cultivable. This restricted our understanding of the dynamics of the intestinal microbiota and how it reacts to different factors. Recent developments in culture independent, molecular based techniques have helped us in better understanding intestinal microbiology and reducing the biases associated with culture dependent techniques. Techniques such as denaturing gradient gel electrophoresis (DGGE), fluorescence in situ hybridisation (FISH), and metagenomic methods based on phylogenetic targets such as 16S rRNA and cpn60 have been employed to characterise the structure of rainbow trout intestinal microbiota (S panggaard, et al. 2000; Huber, et al. 2004; Mansfield, et al. 2010; Navarrete, et al. 2010). These techniques have given valuable insight into the structure of intestinal microbiota but at the same time they do not yield strain level resolution.

Many studies have shown that diet can alter the structure and composition of the intestinal microbiota. It has been suggested that dietary manipulations can be used in a controlled manner to cause changes in the intestinal microbiota in a way that can be beneficial for fish health (Spanggaard, et al. 2001; Aas, et al. 2006; Heikkinen, et al. 2006; Kim, et al. 2006). These approached include the use of dietary additives and antimicrobials (Glencross, et al. 2004; Bakke- 
McKellep, et al. 2007; Dimitroglou, et al. 2009). Correspondingly fewer studies have concentrated on better understanding the effects of seasonal variation (Spanggaard, et al. 2000; Huber, et al. 2004) or the surrounding environment (Hansen, et al. 1999) on the intestinal microbiota of rainbow trout. However, it has been observed that the intestinal microbiota of fish as well as higher vertebrates can be influenced by a shared environment (Roeselers, et al. 2011; Spor, et al. 2011). It is important to study the effect of factors such as shared environment on the creation of a "pan-microbiome" within an experimental system because these factors can influence the interpretation of results of studies designed to determine the effects of diet or environment manipulation on the intestinal microbiome, possibly introducing a normalizing effect that decreases the apparent effect of experimental manipulations.

The objective of this study was to evaluate the extent of sharing of strains of bacteria in the intestinal microbiota of individual fish housed in a recirculating facility over a period of time. We have selected C. maltaromaticum as our target as it is a known constituent of intestinal microbiota of salmonid fish (Ringo, et al. 1995). In our previous study (Mansfield, et al. 2010) based on cpn60 clone library analysis we saw that the most frequently observed sequence was identical to $C$. maltaromaticum and that these sequences were the only sequences which were common to all fish in the study, regardless of diet. We have used a rep-PCR based on GTG $_{5}$ rep PCR primers to discriminate between different strains of C. maltaromaticum. $\mathrm{GTG}_{5}$ rep PCR is a fast and reliable technique which has been used to identify, classify and discriminate between

\section{6}


different bacterial species as well as the strains of the same bacterial species (Gevers, et al. 2001; Svec, et al. 2005; Svec, et al. 2005; Lee, et al. 2011). To our knowledge this is the first study aimed at evaluating the extent to which rainbow trout housed in a shared facility share different strains of the same bacterial species. 


\subsection{Materials and methods:}

\subsubsection{Fish husbandry, diets and sample collection:}

The trials were conducted at the Prairie Aquaculture Research Centre (University of Saskatchewan, Saskatoon, SK), a biologically filtered semiclosed recirculating aquaculture system. Triploid female rainbow trout (Oncorhynchus mykiss), were used for the trials and were acquired from Wild West Steelhead (Lucky Lake, SK). Fish were housed in $360 \mathrm{~L}$ tanks with water temperature adjusted at $15 \pm 1{ }^{\circ} \mathrm{C}$ and the photoperiod was $14 \mathrm{~h}$ light: $10 \mathrm{~h}$ dark. Water quality indicators were closely monitored over the course of the experiment. Fish were acclimatized to the farm environment by housing them for two weeks before the trial on a commercial fishmeal based diet. All instructions and guidelines set by the Canadian Council of Animal Care (CCAC, 2005) were followed throughout the experimental trial (University Committee on Animal Care and Supply Protocol 19980142). All fish were hand-fed to satiety twice daily with diets formulated using different plant ingredients, namely pea meal $(\mathrm{PM})$, soy protein concentrate (SPC), canola meal (CM) and canola protein concentrate (CPC) at different inclusion rates $(0,7.5,15,22.5$ and $30 \%$ of fish meal replaced with the plant ingredient). All diets were fed for a period of 56 days. Experimental diet trials were not conducted simultaneously, but were performed in series as described previously (Chapter 4). At the end of each feeding trial random fish were selected, euthanized and the distal gut contents were collected aseptically.

\section{8}


6.3.2 C. maltaromaticum isolation:

Distal gut contents from individual fish were serially diluted and spread on cresol red thallium acetate sucrose inulin (CTSI) agar (Wasney, et al. 2001). Plates were incubated at $25^{\circ} \mathrm{C}$ for two days and at $8{ }^{\circ} \mathrm{C}$ for an additional two days. Isolated C. maltaromaticum colonies used to inoculate MRS broth and incubated overnight at $35^{\circ} \mathrm{C}$. Glycerol stocks $(15 \% \mathrm{v} / \mathrm{v})$ of C. maltaromaticum were prepared and stored at $-80^{\circ} \mathrm{C}$ until further use. The number of isolates from individual fish and diets fed is shown in Table 6.1. 
Table 6.1 Number of Carnobacterium maltaromaticum strains isolated from distal intestinal contents of rainbow trout fed with different diets.

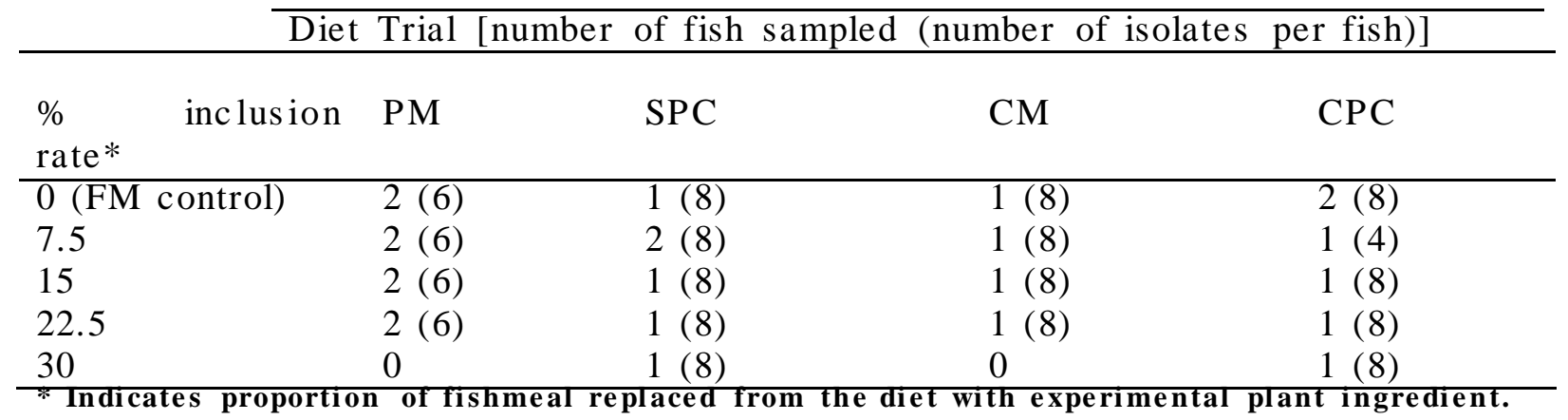




\subsection{3 cpn60 PCR and sequencing:}

Species-specific PCR based on the cpn60 universal target was used to confirm the identity of isolates (Mansfield, et al. 2010). PCR amplifications were performed in a $50 \mu \mathrm{L}$ reaction consisting $5 \mu \mathrm{L}$ of $10 \times$ PCR reaction buffer, $2 \mathrm{mM} \mathrm{MgCl} l_{2}, 0.2 \mathrm{mM}$ each of a dNTP mixture, $0.4 \mu \mathrm{M}$ of each primer (forward: 5'-GCA ATT TGA CCG TGG TTA CC-3' and reverse: 5'-TTA ATA ATG GAC CTT GTT GG-3') and $0.5 \mathrm{U}$ of Platinum Taq DNA polymerase (Invitrogen, Burlington, ON, Canada). Thermocycling parameters were 3 min at $95{ }^{\circ} \mathrm{C}$ followed by 40 cycles of $95{ }^{\circ} \mathrm{C}$ for $30 \mathrm{~s}, 64{ }^{\circ} \mathrm{C}$ for $30 \mathrm{~s}$ and $72{ }^{\circ} \mathrm{C}$ for $30 \mathrm{~s}$ followed by 5 mins of final extension at $72{ }^{\circ} \mathrm{C}$. Amplified PCR product was expected to be $167 \mathrm{bp}$ in length. PCR products were purified and sequenced directly at National Research Council Plant Biotechnology Institute, Saskatoon, SK.

\subsubsection{DNA extraction and $G T G_{5}$ rep PCR:}

Genomic DNA was extracted from overnight broth cultures of $C$. maltaromaticum isolates using a modified salting out procedure (Martin-Platero, et al. 2007). Genomic DNA from a total of 172 C. maltaromaticum strains isolated from different fish fed with different diets was extracted (Table 1). $\mathrm{GTG}_{5}$ rep PCR was performed as described earlier (Versalovic, et al. 1994). PCR was performed in a final volume of $25 \mu \mathrm{L}$ with $10 \%$ (v/v) DMSO. PCR conditions were set as 3 min at $95^{\circ} \mathrm{C}$ as initial denaturation followed by 35 cycles of $30 \mathrm{sec}$ at $95^{\circ} \mathrm{C}$ of denaturation, $1 \mathrm{~min}$ at $40{ }^{\circ} \mathrm{C}$ and $8 \mathrm{mins}$ at $65{ }^{\circ} \mathrm{C}$,

\section{1}


and ended with a final extension of 10 mins at $65{ }^{\circ} \mathrm{C}$. PCR products were visualized on a $1.5 \%$ agarose gel, run for 5 hours at $100 \mathrm{~V}$. Three lanes in each gel (one on each end and one in the center) were assigned for a $1 \mathrm{~kb}$ molecular marker. Gels were stained in TBE buffer containing $10 \mathrm{mg} \mathrm{ml}^{-1}$ ethidium bromide solution and photographed under UV light.

\subsubsection{Cluster analysis:}

$\mathrm{GTG}_{5}$ rep PCR fingerprints were normalized and analyzed using GelCompar II V6.1 software (Applied Maths Inc., Austin, TX). Percent similarity indices of the fingerprints representing individual $C$. maltaromaticum strains were calculated using the Dice coefficient. Dendrograms of the fingerprints were built using the calculated Dice coefficient un-weighted pair group method algorithm (UPGMA). 


\subsection{Results:}

The $\mathrm{GTG}_{5}$ rep PCR fingerprints of 172 C. maltaromaticum isolates from 24 individual fish were generated (4 to 8 isolates per fish)(Table 6.1). GTG 5 rep PCR fingerprints contained from 6 to 23 bands, ranging in size from 500 to 5000 bp.

Cluster analysis of these isolates based on Dice coefficient showed that the minimum percent similarity of the profiles for isolates from individual fish ranged from 68 to $100 \%$ for PM fed fish, 56 to $100 \%$ for SPC, 57 to $100 \%$ for $\mathrm{CM}$ and 79 to $100 \%$ for CPC fed fish (Figure 6.1 to 6.4 respectively). Few pairs or groups of isolates with identical fingerprints were observed: two pairs of identical isolates each for $\mathrm{FM}_{\mathrm{PM}^{-}} 109$ and $\mathrm{PM}-127$, one pair of identical isolates for $\mathrm{FM}_{\mathrm{SPC}}-244$, and one pair of identical isolates each for $\mathrm{FM}_{\mathrm{CM}^{-}} 288$ and $\mathrm{CM}$ 297. Only three groups of more than two identical fingerprints were observed, including 4 identical isolates from PM-128, 7 identical isolates from $\mathrm{FM}_{\mathrm{CPC}}-334$, 4 identical isolates from $\mathrm{FM}_{\mathrm{CPC}^{-}} 336$. 


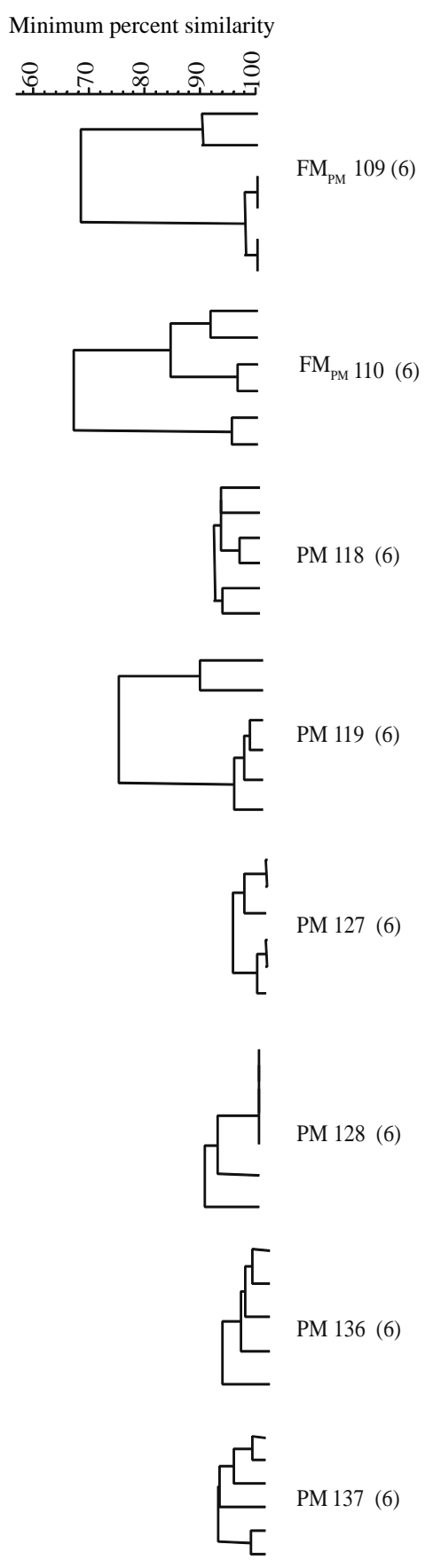

Figure 6.1 Dendograms derived from $\mathrm{GTG}_{5}$ rep PCR fingerprints of $C$. maltaromaticum isolates from distal gut contents of individual fish reared during the PM diet trial. Labels indicate fish number. Numbers in parentheses indicate the number of strains isolated from that particular fish. 


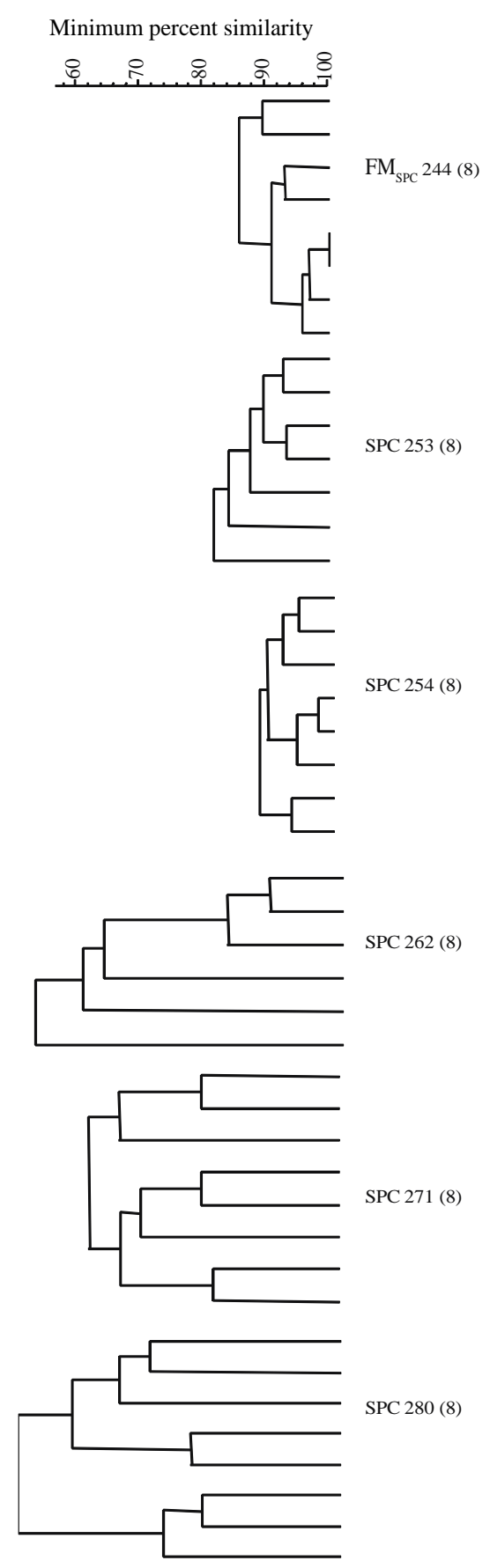

Figure 6.2 Dendograms derived from $\mathrm{GTG}_{5}$ rep PCR fingerprints of $C$. maltaromaticum isolates from distal gut content of individual fish reared during the SPC diet trial. Labels indicate fish number. Numbers in parentheses indicate the number of strains isolated from that particular fish.

\section{5}




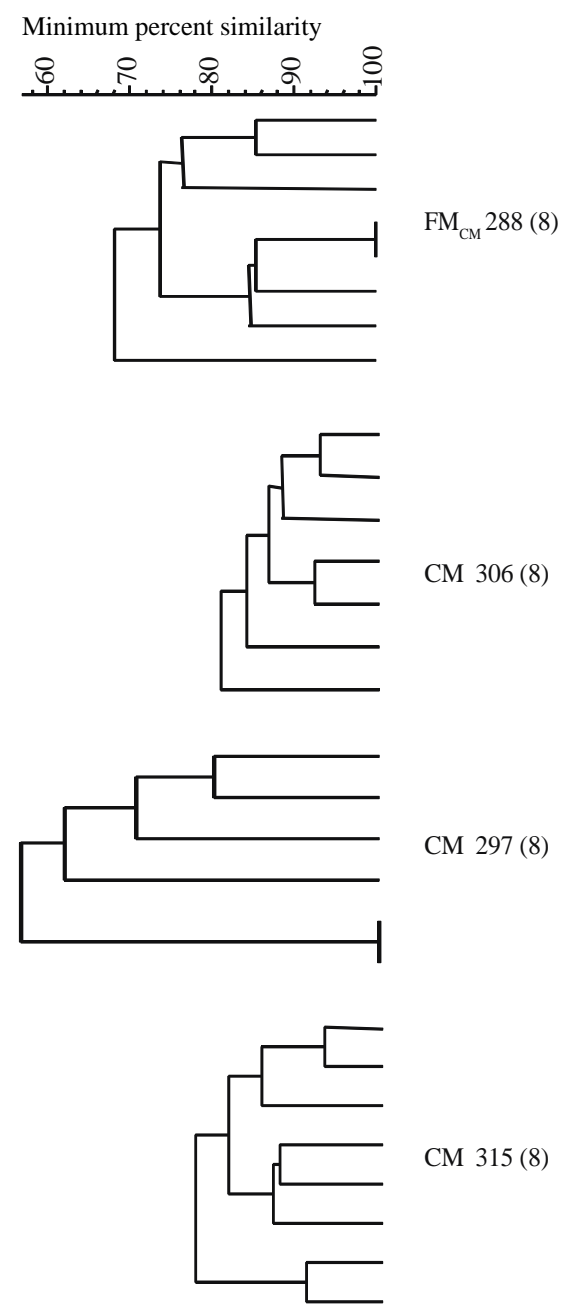

Figure 6.3 Dendograms derived from $\mathrm{GTG}_{5}$ rep PCR fingerprints of $C$. maltaromaticum isolates from distal gut content of individual fish reared during the CM diet trial. Labels indicate fish number. Numbers in parentheses indicate the number of strains isolated from that particular fish. 


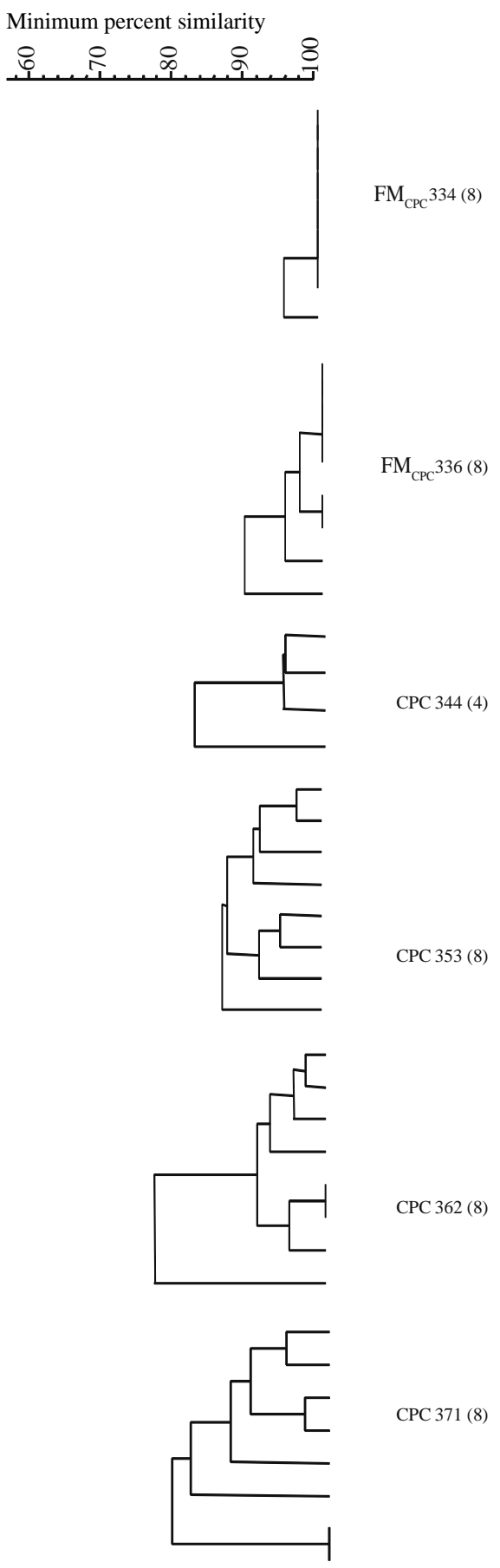

Figure 6.4 Dendograms derived from $\mathrm{GTG}_{5}$ rep PCR fingerprints of $C$. maltaromaticum isolates from distal gut content of individual fish reared during the CPC diet trial. Labels indicate fish number. Numbers in parentheses indicate the number of strains isolated from that particular fish. 
The observed strain level variation was unexpectedly high, so 12 isolates across a range of minimum similarity values were subjected to cpn60 based sequence analysis using species specific PCR assays developed for $C$. maltaromaticum to confirm their identity (Mansfield, et al. 2010). cpn60 sequences from all 12 isolates were at least $96 \%$ identical in nucleotide sequence to the $C$. maltaromaticum ATCC 27865 type strain reference sequence in cpnDB.

Cluster analys is using the Dice coefficient was also conducted to understand genetic variation among $C$. maltaromaticum isolates collected during the same time period (i.e. same diet trial). GTG $_{5}$ rep PCR profiles of all $C$. maltaromaticum strains isolated from fish fed during each experimental diet trial were clustered together to determine if they clustered based on individual fish of origin (Figure 6.5). A lower overall minimum percent similarity was observed among isolates from the SPC (45\%) and CM (30\%) trials relative to either PM $(69 \%)$ or CPC $(75 \%)$.

In all trial periods, for the majority of fish sampled, most of the isolates cluster together, closer to each other than to isolates from other fish (Figure 6.5). The least fish-based clustering was observed in the PM trial where for only half (4/8) of the fish, the majority (3/6 or 4/6) isolates cluster together (Figure 6.5A). Isolates from the SPC trial were the most clustered based on individual fish of origin, with the majority of isolates (4/8 to 8/8) clustering together for all (6/6) fish sampled (Figure 6.4B). Isolates from $3 / 4$ and $4 / 6$ 
individual fish clustered together in the CM and CPC trials respectively (Figure 6.5C and 6.5D). 


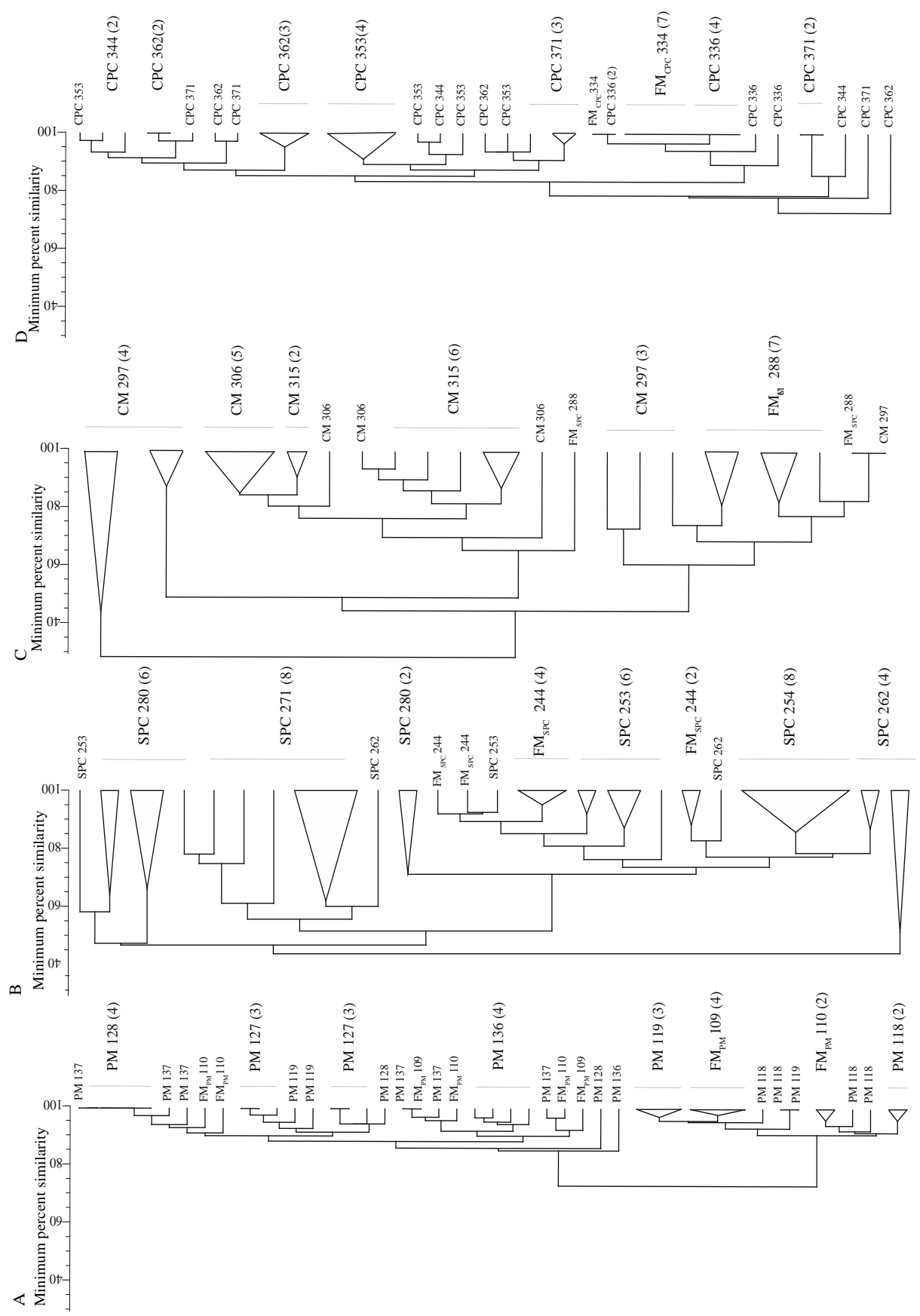

Figure 6.5 Dendograms derived from $\mathrm{GTG}_{5}$ rep PCR fingerprints of $C$. maltaromaticum isolates from distal gut contents of all fish reared during (A) PM, (B) SPC, (C) CM, and (D) CPC diet trials. Dendograms were derived using Dice coefficient and the UPGMA clustering method. Labels indicate fish number. Numbers in parentheses indicate the number of strains isolated from that particular fish 


\subsection{Discussion}

Results presented in this study were a part of a trial designed to evaluate the effects of replacing fishmeal in traditional aquaculture feed with plant ingredients on the intestinal microbiota of rainbow trout. This study occurred over the course of one year during which fish were maintained in a biologically filtered indoor recirculating aquaculture facility. Previous studies have shown that the surrounding environment can influence the composition of intestinal microbiota (Hansen, et al. 1999; Gomez, et al. 2008; Perez, et al. 2010). Since the facility was well monitored for temperature fluctuations, $\mathrm{pH}$ of the water, and conditions were maintained to keep the fish at minimal stress levels, it is unlikely that differences observed in the intestinal microbial composition of fish on different diets were influenced by these factors. It has also been reported that establishment of normal intestinal microbiota of fish reared in an aquaculture facility for an extended period of time can be influenced by environmental bacteria (Sugita, et al. 1988; Cahill. 1990). Given the recirculation of water among tanks in the facility, each tank housing fish on different diets, we sought to investigate the extent to which a "pan-microbiome" might be shared among fish, regardless of their diet, since this could have an influence on the degree of diet effects that could be detected with this type of experimental design. If a pan-microbiome was present and the intestinal and fecal microbiomes of fish were influencing fish downstream in the recircultating facility, we would expect to see some identical strains isolated from different individuals, and a random

\section{1}


clustering of isolates from different fish, consistent with a lack of fish-based clustering of isolates.

$\mathrm{GTG}_{5}$ rep PCR has been widely used for identification and discrimination of different beneficial as well as pathogenic bacteria (Sechi, et al. 1999; Svec, et al. 2005; Mohapatra, et al. 2008; Braem, et al. 2011; Svec, et al. 2011). GTG5 rep PCR is based on using an oligonucleotide primer which simultaneously amplifies repetative DNA elements in the bacterial genome (Versalovic, et al. 1994). GTG 5 rep PCR has been used previously to identify different lactic acid bacteria (Vasilopoulos, et al. 2010), but strain level differentiation of $C$. maltaromaticum using $\mathrm{GTG}_{5}$ rep PCR has not been reported to the authors' knowledge.

Cluster analysis of $\mathrm{GTG}_{5}$ rep PCR profiles of C. maltaromaticum strains isolated from the intestinal contents of individual fish demonstrated a considerable level of genome structure diversity, with minimum similarities between profiles ranging from 56-100\% (Figure 6.1-4). The amount of diversity we observed was consistent with previous studies evaluating diversity of $C$. maltaromaticum strains associated with food spoilage (Vasilopoulos, et al. 2010). This approach revealed diversity in the microbiome at a strain level, not detected by techniques employed previously in this thesis.

Clustering of all C. maltaromaticum strains isolated from the same time period (same diet trial) showed that strains frequently clustered by the fish of origin, suggesting each individual fish has its own microbiome that is evolving on its own, independent of other fish in the same environment. These results 
support the suggestion that there is little strain sharing among fish housed in the recirculating facility at the same time. The feeding trial was extended over a period of 12 months starting with the PM diet trial followed with SPC and CM diets and ending with CPC diet. The least fish-based clustering of $C$. maltaromaticum strains was observed in the PM trial (Figure 6.5), with more prominent clustering by fish for isolates collected in the later feeding trials. This trend suggests that as time passes, individual fish develop their own microbiomes which become progressively more distinct from other fish reared in the same environment.

The intestinal microbiomes of rainbow trout have been shown to share common bacterial species regardless of diet, although their proportional abundances may be affected by diet (Huber, et al. 2004; Dimitroglou, et al. 2009; Mansfield, et al. 2010). A core microbiome was also described for zebra fish in a recent study looking at intestinal microbiota of farmed and wild zebra fish from different geographic locations (Roeselers, et al. 2011). Characterisation of the intestinal microbiota of individual fish from this trial showed similar results in that $C$. maltaromaticum was isolated from all fish sampled and thus comprises part of a core microbiome for these rainbow trout.

Characterisation of fish intestinal microbiota by culture dependent and independent methods has shown that fish intestinal microbiota is composed of certain bacterial classes, particularly the gamma subclass of Proteobacteria and Firmicutes (Huber, et al. 2004; Merrifield, et al. 2010; Navarrete, et al. 2010). This suggests that the fish intestinal environment is better suited to only few 
bacterial classes, C. maltaromaticum been one of them. Based on cases where isolates from multiple fish in the same diet group were examined (PM trial, 2 fish per inclusion rate) it was apparent that those fish did not share identical strains, indicating that the core microbiome does not exist beyond the species level. These clustering patterns suggest that the intestinal microbiome of a fish, and the bacteria shed in its feces, do not "contaminate" the microbiome fish downstream in the recirculating facility.

To summarize our results, we found $C$. maltaromaticum as a constituent of the intestinal microbiota of all fish fed with different plant based ingredients. $\mathrm{GTG}_{5}$ rep PCR resolved intraspecies diversity within C. maltaromaticum and as the trial progressed, fish to fish variation was more evident with individual fish harbouring strains unique to that individual fish and more closely related to each other than to isolates from other fish. This is encouraging for future diet trials exploiting recirculating aquaculture facilities since it suggests that th is housing design does not lead to diet-independent development of intestinal microbiota and a corresponding reduction in our ability to detect diet-associated changes in the microbiome. 


\section{Chapter 7 Summary and conclusions}

\subsection{Summary:}

Plant ingredients are used to formulate aqua-feeds with the goal of reducing reliance of the aquaculture industry on FM as the primary protein source, as production of FM has decreased globally making it an expensive and non-sustainable resource (Tacon, et al. 2008). Replacement of FM with plant ingredients has been reported to result in decreased growth performance and intestinal inflammation in carnivorous fish and these negative effects on the health of fish have been associated with the anti-nutritional factors present in the plant ingredients (Drew, et al. 2007). The critical role of intestinal microbiota in intestinal development, protection from pathogens and digestion in fish suggests that the negative effects of plant ingredients on intestinal physiology of salmonid fish are likely mediated at least partly through alteration in the structure of intestinal microbiota (Gomez, et al. 2008; Merrifield, et al. 2010). A critical first step in validating this hypothesis is to demonstrate that plant ingredient based diets are associated with distinct intestinal microbial profiles compared to a "gold standard" FM diet. The studies described in this thesis were conducted to use a combination of culture-dependent and cultureindependent methods to create a comprehensive inventory of the rainbow trout intestinal microbiome, to determine the structure of the intestinal microbiome of trout fed with different diets formulated using plant ingredients (soybean, canola and peas) at two different processing levels, and to investigate fish to fish variation in intestinal microbiome structure. 
In the first trial (Chapter 3) we established a comprehensive sequence database which gave an overall description of the rainbow trout intestinal microbiome using cpn60 clone library analysis, and qRT-PCR which evaluated abundance of specific bacterial targets in individual fish. In addition to supporting the hypothesis that diet effects on the fish intestine include indirect effects mediated through modifications of the intestinal microbiota, the results also indicated the importantance of characterising the intestinal microbiome of individual fish rather than pooled samples to improve the identification of significant differences in a complex environment with substantial individual to individual variation.

One of the main aims of this project was to characterise and define a "healthy intestinal microbiome", providing a reference point for future investigations of new ingredients as suitable and sustainable sources of protein. It was speculated that it might be possible to identify a subset of informative, indicator species, whose abundance is representative of the healthy microbiome. In this study we used different culture and culture independent tools resulting in descriptions of the trout intestinal microbiome over a wide spectrum of resolution, from the overall microbiome fingerprint to strain level resolution of cultured bacteria. Irrespective of the diets fed and the approach to characterize the intestinal microbiota, it was observed that gamma Proteobacteria and Firmicutes were the dominant phyla of the trout distal intestinal microbiome, in accordance with previous studies (Spanggaard, et al. 2000; Huber, et al. 2004; Kim, et al. 2007; Navarrete, et al. 2009; Navarrete, et al. 2010). There was

\section{6}


considerable diversity at the strain level between inidividual animals and substantial variation in the abundance of bacterial species between individual fish. These results are in accordance with previous studies showing differences in the relative abundance of bacterial phylotypes (Benson, et al. 2010) in animals with similar host genotype (Ley, et al. 2005; Wen, et al. 2008) and shared environment, and indicate that information generated at such high resolution is not useful for diagnostic purposes.

More consistency was observed at higher taxonomic levels. Data generated using clone library analysis and pyrosequencing suggested that the intestinal microbiomes of fish fed with diets based on plant ingredients had a higher Firmicutes:Proteobacteria ratios and showed greater overall richness and diversity than microbiomes of fish fed FM diets. These consistent results, supplemented with DGGE analysis that showed consistent differences between microbial profiles of fish fed plant ingredients and the FM control diet, indicate that these techniques, which describe the intestinal microbiome at a lower level of resolution, could be very useful for detecting diet associated disturbance of this microbial community.

It has been shown in previous studies that processing of plant meals to protein concentrates results in superior growth performance (Borgeson, et al. 2006; Drew, et al. 2007). Our observations suggest a consistent difference between the structures of intestinal microbiota of fish associated with the FM control and experimental diets. These differences are smaller when fish are fed with protein concentrate diets based on the same plant ingredients. This strategy 
to reduce the antinutritional factors from the meals by processing them further and making the protein in the diets more accessible to the fish should be exploited by the industry to reduce its use of FM and boost sustainability and growth of this industry, although additional work may be required to make the production of protein concentrates economically viable. Growth analysis of fish showed that $\mathrm{SBM}$ and $\mathrm{CM}$ based diets had a negative effect, whereas diets based on the concentrate version of the plant ingredients had no effect on the growth. (Collins, et al. 2011). Furthermore, the SBM diet was associated with an inflammatory response in intestinal tissue of the fish. CM and all other diets did not show any correlation with inflammation (Mansfield, G.S. et al., unpublished data). Association of negative growth and inflammation with meal based diets in rainbow trout may be partially be an effect of direct as sociation of the diet or an indirect effect of alteration of the gut microbiota. Comparing and relating findings from this thesis to the host reponse and growth outcome data suggest that replacement of up to $30 \%$ of FM from a aquafeed with a plant protein concentrate ingredient can be achieved with no negative effects on growth. Use of locally grown and processed plant ingredients in aqua feed formulation such as canola protein concentrate, will provide Saskatchewan farmers with an alternative market.

Space constraints at the Prairie Aquaculture Research Centre necessitated an experimental design in which the major growth trial described in Chapter 4 was conducted over a year, rather than testing all diets simultaneously. An unexpected outcome of this was the observation of changes in the FM diet 
associated microbiome over this time period. This result draws attention to the fact that fish age and length of time in the farm environment are potentially significant experimental variables. To better understand the effects of diets on the intestinal microbiota and permit direct comparison of microbial profiles associated with different ingredients, it would be desirable to characterise the intestinal microbiota of fish at different ages and stages of maturity. The intestinal microbiota of individual fish would ideally be profiled at the beginning of the trial, and at multiple time points during the trial. This would help in understanding the succession and dynamics of the intestinal microbiome in response to the experimental diet.

Finally, it is imperative that the findings are correlated to the growth and the health of the fish. Results from studies aimed at characterising the intestinal microbiome are most informative only when they are related to the physiological outcomes of the experimental diets. Fortunately, the opportunity to accomplish this is at hand. The work described in this thesis is part of a larger, interdisciplinary research program and the results described here will be combined with additional growth, health and nutritional parameters measured by collaborating researchers during the described feeding trials in an effort to more fully describe the host-diet-microbiome interactions that determine the utility of any novel diet formulation. 


\subsection{Conclusions:}

1. Firmicutes, especially lactic acid bacteria, and gamma Proteobacteria dominate the intestinal microbiome of farmed rainbow trout. The abundance ratio of Firmicutes to Proteobacteria was consistently higher in fish fed a plant ingredient diet compared to those fed the FM control diet.

2. The intestinal microbiota of fish fed with plant ingredient based diets is structurally different from that of fish fed an FM based diet. However, this difference was less evident for plant protein concentrate diets compared to meal based diets.

3. Substantial fish to fish variation in the abundance of individual bacterial species was observed, and plant ingredient associated microbial profiles were not generally reproducible at the level of individual bacterial species in replicate feeding trials.

4. Individual fish host unique collections of strains of C. maltaromaticum, more closely related to each other than to isolates from other fish, suggesting that although the fish share a core microbiome at the species level, each animal's microbiome is unique at the subspecies level. 


\section{Chapter 8: References}

Aas TS, Hatlen B, Grisdale-Helland B, Terjesen BF, Bakke-McKellep AM, Helland SJ (2006). Effects of diets containing a bacterial protein meal on growth and feed utilisation in rainbow trout (Oncorhynchus mykiss). Aquacul 261:357-368.

Aksnes A, Hope B, Hostmark O, Albrektsen S (2006). Inclusion of size fractionated fish hydrolysate in high plant protein diets for Atlantic cod, Gadus morhua. Aquacul 261:1102-1110.

Al-Maghazachi SJ, Ray G (1984). The developmental stages of larval turbot, Scophthalmus maximus (L.). J Exp Mar Biol Ecol 82:35-51.

Altschul SF, Gish W, Miller W, Myers EW, Lipman DJ (1990). Basic local alignment search tool. J Mol Biol 215:403-410.

Amend AS, Seifert KA, Bruns TD (2010). Quantifying microbial communities with 454 pyrosequencing: does read abundance count? Mol Ecol 19:5555-5565.

Anderson RL, Wolf WJ (1995). Compositional changes in trypsin inhibitors, phytic acid, saponins and isoflavones related to soybean processing. J Nutr 125:581-588.

Atalah E, Cruz CMH, Izquierdo MS, Rosenlund G, Caballero MJ, Valencia A, et al. (2007). Two microalgae Crypthecodinium cohnii and Phaeodactylum 
tricornutum as alternative source of essential fatty acids in starter feeds for seabream (Sparus aurata). Aquacul 270:178-185.

Austin B, Al-Zahrani AMJ (1988). The effect of antimicrobial compounds on the gastrointestinal microflora of rainbow trout, Salmo gairdneri Richardson. J Fish Biol 33:1-14.

Baeverfjord G, Krogdahl A (1996). Development and regression of soybean meal induced enteritis in Atlantic salmon, Salmo salar L., distal intestine: a comparison with the intestines of fasted fish. J Fish Dis 19:375-387.

Bairagi A, Ghosh KS, Sen SK, Ray AK (2002). Enzyme producing bacterial flora isolated from fish digestive tracts. Aquacul Int 10:109-121.

Bakke-McKellep AM, Penn MH, Salas PM, Refstie S, Sperstad S, Landsverk T, et al. (2007). Effects of dietary soyabean meal, inulin and oxytetracycline on intestinal microbiota and epithelial cell stress, apoptosis and proliferation in the teleost Atlantic salmon (Salmo salar L.). Br J Nutr 97:699-713.

Bakke-McKellep AM, Press CM, Baeverfjord G, Krogdahl A, Landsverk T (2000). Changes in immune and enzyme histochemical phenotypes of cells in the intestinal mucosa of Atlantic salmon, Salmo salar L., with soybean mealinduced enteritis. J Fish Dis 23:115-127. 
Balcázar JL, Vendrell D, De Blas I, Ruiz-Zarzuela I, Muzquiz JL, Girones O (2008). Characterization of probiotic properties of lactic acid bacteria isolated from intestinal microbiota of fish. Aquacul 278:188-191.

Benson AK, Kelly SA, Legge R, Ma F, Low SJ, Kim J, et al. (2010). Individuality in gut microbiota composition is a complex polygenic trait shaped by multiple environmental and host genetic factors. Proc Natl Acad Sci U S A 107:18933-18938.

Bent SJ, Forney LJ (2008). The tragedy of the uncommon: understanding limitations in the analysis of microbial diversity. ISME $J$ 2:689-695.

Bindelle J, Pieper R, Montoya CA, Van Kessel AG, Leterme P (2011). Nonstarch polysaccharide-degrading enzymes alter the microbial community and the fermentation patterns of barley cultivars and wheat products in an in vitro model of the porcine gastrointestinal tract. FEMS Microbiol Ecol 76:553-563.

Blaut M (2011). Ecology and Physiology of the Intestinal Tract. Curr Top Microbiol Immunol Epub.

Borgeson TL, Racz VJ, Wilkie DC, White LJ, Drew MD (2006). Effect of replacing fishmeal and oil with simple or complex mixtures of vegetable ingredients in diets fed to Nile tilapia (Oreochromis niloticus). Aquacult Nut 12:141-149. 
Braem G, De Vliegher S, Supre K, Haesebrouck F, Leroy F, De Vuyst L (2011). (GTG)5-PCR fingerprinting for the classification and identification of coagulase-negative Staphylococcus species from bovine milk and teat apices: a comparison of type strains and field isolates. Vet Microbiol 147:67-74.

Brosius J, Palmer ML, Kennedy PJ, Noller HF (1978). Complete nucleotide sequence of a $16 \mathrm{~S}$ ribosomal RNA gene from Escherichia coli. Proc Natl Acad Sci U S A 75:4801-4805.

Brugman S, Liu KY, Lindenbergh-Kortleve D, Samsom JN, Furuta GT, Renshaw SA, et al. (2009). Oxazolone-induced enterocolitis in zebrafish depends on the composition of the intestinal microbiota. Gastroenterology 137:1757-1767.

Brunvold L, Sandaa R, Mikkelsen H, Welde E, Bleie H, Bergh O (2007). Characterisation of bacterial communities associated with early stages of intensively reared cod (Gadus morhua) using Denaturing Gradient Gel Electrophoresis (DGGE). Aquacul 272:319-327.

Burr G, Hume M, Neill WH, Gatlin III DM (2008). Effects of prebiotics on nutrient digestibility of a soybean-meal-based diet by red drum Sciaenops ocellatus (Linnaeus). Aquacult Res 39:1680-1686.

Cahill MM (1990). Bacterial flora of fishes: A review. Microb Ecol 19:21-41. 
Cai Y, Benno Y, Nakase T, Oh TK (1998). Specific probiotic characterization of Weissella hellenica DS-12 isolated from flounder intestine. J Gen Appl Microbiol 44:311-316.

Caton IR, Schneegurt MA (2011). Culture-independent analysis of the soil bacterial assemblage at the Great Salt Plains of Oklahoma. J Basic Microbiol Epub.

Chaban B, Links MG, Hill JE (2012). A Molecular Enrichment Strategy Based on cpn60 for Detection of Epsilon-Proteobacteria in the Dog Fecal Microbiome. Microb Ecol 63:348-357.

Cho CY, Cowey CB, Watanabe $\mathrm{T}$ (1985). Finfish Nutrition in Asia: Methodological Approaches to Research and Development. International Development Research Centre, Ottawa, Canada. Nature.

Collins SA, Desai AR, Mansfield GS, Hill JE, Van Kessel AG, Drew MD (2011). The effect of increasing inclusion rates of soybean, pea and canola meals and their protein concentrates on the growth of rainbow trout: Concepts in diet formulation and experimental design for ingredient evaluation. Aquacul Epub.

Cunliffe M, Upstill-Goddard RC, Murrell JC (2011). Microbiology of aquatic surface microlayers. FEMS Microbiol Rev 35:233-246. 
Cury P, Bakun A, Crawfors RJM, Jarre A, Quiñones RA, Shannon LJ, et al. (2000). Small pelagics in upwelling systems: Patterns of interaction and structural changes in "wasp-waist" ecosystems. ICES J Mar Sci 57:603-618.

Dabrowski K, Poczyczynski P, Köck G, Berger B (1989). Effect of partially or totally replacing fish meal protein by soybean meal protein on growth, food utilization and proteolytic enzyme activities in rainbow trout (Salmo gairdneri). New in vivo test for exocrine pancreatic secretion. Aquacul 77:29-49.

Denev S, Staykov Y, Moutafchieva R, Beev G (2009). Microbial ecology of the gastrointestinal tract of fish and the potential application of probiotics and prebiotics in finfish aquaculture. Int Aqua Res 1:1-29.

Desai AR, Musil KM, Carr AP, Hill JE (2009). Characterization and quantification of feline fecal microbiota using cpn60 sequence-based methods and investigation of animal-to-animal variation in microbial population structure. Vet Microbiol 137:120-128.

Dimitroglou A, Merrifield DL, Moate R, Davies SJ, Spring P, Sweetman J, et al. (2009). Dietary mannan oligosaccharide supplementation modulates intestinal microbial ecology and improves gut morphology of rainbow trout, Oncorhynchus mykiss (Walbaum). J Anim Sci 87:3226-3234.

Drew MD, Borgeson TL, Thiessen DL (2007). A review of processing of feed ingredients to enhance diet digestibility in finfish. Anim Feed Sci Technol 138:118-136. 
Dumonceaux TJ, Hill JE, Hemmingsen SM, Van Kessel AG (2006a). Characterization of intestinal microbiota and response to dietary virginiamycin supplementation in the broiler chicken. Appl Environ Microbiol 72:2815-2823.

Dumonceaux TJ, Hill JE, Briggs SA, Amoako KK, Hemmingsen SM, Van Kessel AG (2006b). Enumeration of specific bacterial populations in complex intestinal communities using quantitative PCR based on the chaperonin-60 target. $J$ Microbiol Methods 64:46-62.

Dumonceaux TJ, Hill JE, Briggs SA, Amoako KK, Hemmingsen SM, Van Kessel AG (2006c). Enumeration of specific bacterial populations in complex intestinal communities using quantitative PCR based on the chaperonin-60 target. Journal of Microbiological Methods 64:46-62.

Eckert KA, Kunkel TA (1991). DNA polymerase fidelity and the polymerase chain reaction. PCR Methods Appl 1:17-24.

Egidius E (1987). Vibriosis: Pathogenicity and pathology. A review. Aquacul 67:15-28.

Elson CO, Cong Y, McCracken VJ, Dimmitt RA, Lorenz RG, Weaver CT (2005). Experimental models of inflammatory bowel disease reveal innate, adaptive, and regulatory mechanisms of host dialogue with the microbiota. Immunol Rev 206:260-276. 
Erijman L, Figuerola EL, Guerrero LD, Ayarza JM (2011). Impact of the recent advances in the analysis of microbial communities on the control of the wastewater treatment process. Rev Argent Microbiol 43:127-135.

Espe M, Lemme A, Petri A, El-Mowafi A (2006). Can Atlantic salmon (Salmo salar) grow on diets devoid of fish meal? Aquacul 255:255-262.

FAO I (2010). The State of World Fisheries and Aquaculture. 2010 (SOFIA).

FAO I (2000). The State of World Fisheries and Aquaculture - 2000 (SOFIA).

Fischer SG, Lerman LS (1983). DNA fragments differing by single base-pair substitutions are separated in denaturing gradient gels: correspondence with melting theory. Proc Natl Acad Sci U S A 80:1579-1583.

Fjellheim AJ, Playfoot KJ, Skjermo J, Vadstein O (2011). Inter-individual variation in the dominant intestinal microbiota of reared Atlantic cod (Gadus morhua L.) larvae. Aquacult Res Epub.

Forster I (1999). A note on the method of calculating digestibility coefficients of nutrients provided by single ingredients to feeds of aquatic animals. Aquacult Nutr 5:143-145.

Francis G, Makkar HPS, Becker K (2001). Antinutritional factors present in plant-derived alternate fish feed ingredients and their effects in fish. Aquacul 199:197-227. 
Gatlin DM, Barrows FT, Brown P, Dabrowski K, Gaylord TG, Hardy RW, et al. (2007). Expanding the utilization of sustainable plant products in aquafeeds: a review. Aquacult Res 38:551-670.

Gevers D, Huys G, Swings J (2001). Applicability of rep-PCR fingerprinting for identification of Lactobacillus species. FEMS Microbiol Lett 205:31-36.

Glencross BD, Carter CG, Duijster N, Evans DR, Dods K, McCafferty P, et al. (2004). A comparison of the digestibility of a range of lupin and soybean protein products when fed to either Atlantic salmon (Salmo salar) or rainbow trout (Oncorhynchus mykiss). Aquacul 237:333-346.

Gomes EF, Rema P, Kaushik SJ (1995). Replacement of fish meal by plant proteins in the diet of rainbow trout (Oncorhynchus mykiss): digestibility and growth performance. Aquacul 130:177-186.

Gomez GD, Balcazar JL (2008). A review on the interactions between gut microbiota and innate immunity of fish. FEMS Immunol Med Microbiol 52:145154.

Hagi T, Tanaka D, Iwamura Y, Hoshino T (2004). Diversity and seasonal changes in lactic acid bacteria in the intestinal tract of cultured freshwater fish. Aquacul 234:335-346. 
Hajen WE, Higgs DA, Beames RM, Dosanjh BS (1993). Digestibility of various feedstuffs by post-juvenile chinook salmon (Oncorhynchus tshawytscha) in sea water. 2. Measurement of digestibility. Aquaculture 112:333-348.

Hamady M, Lozupone C, Knight R (2010). Fast UniFrac: facilitating highthroughput phylogenetic analyses of microbial communities including analys is of pyrosequencing and PhyloChip data. ISME J 4:17-27.

Hanauer SB (2004). Update on the etiology, pathogenesis and diagnosis of ulcerative colitis. Nat Clin Pract Gastroenterol Hepatol 1:26-31.

Hansen AC, Karlsen O, Rosenlund G, Rimbach M, Hmere GI (2007). Dietary plant protein utilization in Atlantic cod, Gadus morhua L. Aquacult Nutr 13:200-215.

Hansen GH, Olafsen JA (1999). Bacterial Interactions in Early Life Stages of Marine Cold Water Fish. Microb Ecol 38:1-26.

Hardy WR (2010). Utilization of plant proteins in fish diets: effects of global demand and supplies of fishmeal. Aquacult Res 41:770-776.

Heikkinen J, Vielma J, Kemiläinen O, Tiirola M, Eskelinen P, Kiuru T, et al. (2006). Effects of soybean meal based diet on growth performance, gut histopathology and intestinal microbiota of juvenile rainbow trout (Oncorhynchus mykiss). Aquacul 261:259-268. 
Hendricks JD (2002). Adventious toxins. In: Halver, J. E. (ed). Fish Nutrition 3rd editions. In:, pp 601-649.

Hill JE, Fernando WM, Zello GA, Tyler RT, Dahl WJ, Van Kessel AG (2010). Improvement of the representation of bifidobacteria in fecal microbiota metagenomic libraries by application of the cpn60 universal primer cocktail. Appl Environ Microbiol 76:4550-4552.

Hill JE, Town JR, Hemmingsen SM (2006). Improved template representation in cpn60 polymerase chain reaction (PCR) product libraries generated from complex templates by application of a specific mixture of PCR primers. Environ Microbiol 8:741-746.

Hill JE, Goh SH, Money DM, Doyle M, Li A, Crosby WL, et al. (2005a). Characterization of vaginal microflora of healthy, nonpregnant women by chaperonin-60 sequence-based methods. Am J Obstet Gynecol 193:682-692.

Hill JE, Hemmingsen SM, Goldade BG, Dumonceaux TJ, Klassen J, Zijlstra RT, et al. (2005b). Comparison of ileum microflora of pigs fed corn-, wheat-, or barley-based diets by chaperonin-60 sequencing and quantitative PCR. Appl Environ Microbiol 71:867-875.

Hill JE, Penny SL, Crowell KG, Goh SH, Hemmingsen SM (2004). cpnDB: a chaperonin sequence database. Genome Res 14:1669-1675. 
Holben WE, Williams P, Gilbert MA, Saarinen M, Särkilahti LK, Apajalahti JH (2002). Phylogenetic analysis of intestinal microflora indicates a novel Mycoplasma phylotype in farmed and wild salmon. Microb Ecol 44:175-185.

Hooper LV, Gordon JI (2001). Commensal host-bacterial relationships in the gut. Science 292:1115-1118.

Hovda MB, Fontanillas R, Mcurk C, Obach A, Rosnes JT (2011). Seasonal variations in the intestinal microbiota of farmed Atlantic salmon (Salmo salar L.). Aquacult Res Epub.

Hovda MB, Lunestad BT, Fontanillas R, Rosnes JT (2007). Molecular characterisation of the intestinal microbiota of farmed Atlantic salmon (Salmo salar L.). Aquaculture, 272:581-588.

Huber I, Spanggaard B, Appel KF, Rossen L, Nielsen T, Gram L (2004). Phylogenetic analysis and in situ identification of the intestinal microbial community of rainbow trout (Oncorhynchus mykiss, Walbaum). J Appl Microbiol 96:117-132.

Hugenholtz P, Tyson GW (2008). Microbiology: metage nomics. Nature 455:481483.

Hummelen R, Macklaim JM, Bisanz JE, Hammond JA, McMillan A, Vongsa R, et al. (2011). Vaginal microbiome and epithelial gene array in post-menopausal women with moderate to severe dryness. PLoS One 6:Epub. 
Janczyk P, Pieper R, Smidt H, Souffrant WB (2007). Changes in the diversity of pig ileal lactobacilli around weaning determined by means of $16 \mathrm{~S}$ rRNA gene amplification and denaturing gradient gel electrophoresis. FEMS Microbiol Ecol 61:132-140.

Juul-Madsen HR, Glamann J, Madsen HO, Simonsen M (1992). MHC class II beta-chain expression in the rainbow trout. Scand J Immunol 35:687-694.

Kapetanovic D, Kurtovic B, Teskeredzic E (2005). Differences in Bacterial Population in Rainbow Trout (Oncorhynchus mykiss Walbum) Fry after Transfer from Incubator to Pools. Food Technol Biotechnol 43:189-193.

Kaushik S (1998). Application of the recommendations on vitamin requirements of finfish by NRC (1993) to salmonids and sea bass using practical and purified diets. Aquacul 161:463-474.

Kaushik SJ, Covès D, Dutto G, Blanc D (2004). Almost total replacement of fish meal by plant protein sources in the diet of a marine teleost, the European seabass, Dicentrarchus labrax. Aquacul 230:391-404.

Kim DH, Brunt J, Austin B (2007). Microbial diversity of intestinal contents and mucus in rainbow trout (Oncorhynchus mykiss). J Appl Microbiol 102:16541664. 
Kim DH, Austin B (2006). Cytokine expression in leucocytes and gut cells of rainbow trout, Oncorhynchus mykiss Walbaum, induced by probiotics. Vet Immunol Immunopathol 114:297-304.

Kim KI, Grimshaw TW, Kayes TB, Amundson CH (1992a). Effect of fasting or feeding diets containing different levels of protein or amino acids on the activities of the liver amino acid-degrading enzymes and amino acid oxidation in rainbow trout. Aquacul 107:89-105.

Kim KI, Kayes TB, Amundson CH (1992b). Requirements for lysine and arginine by rainbow trout (Oncorhynchus mykiss). Aquacul 106:333-344.

Kim KI, Kayes TB, Amundson CH (1991). Purified diet development and reevaluation of the dietary protein requirement of fingerling rainbow trout (Oncorhynchus mykiss). Aquacul 96:57-67.

Konstantinov SR, Zhu WY, Williams BA, Tamminga S, Vos WM, Akkermans AD (2003). Effect of fermentable carbohydrates on piglet faecal bacterial communities as revealed by denaturing gradient gel electrophoresis analys is of 16S ribosomal DNA. FEMS Microbiol Ecol 43:225-235.

Kopczynski ED, Bateson MM, Ward DM (1994). Recognition of chimeric smallsubunit ribosomal DNAs composed of genes from uncultivated microorganisms. Appl Environ Microbiol 60:746-748. 
Krogdahl A, Bakke-Mckellep A, Roed K, Baeverfjord G (2000). Feeding Atlantic salmon Salmo salar L. soybean products: effects on disease resistance (furunculosis), and lysozyme and $\operatorname{IgM}$ levels in the intestinal mucosa. Aquaculture Nutrition 6:77-84.

Krogdahl A, Penn M, Thorsen J, Refstie S, Bakke-McKellep AM (2010). Important antinutrients in plant feedstuffs for aquaculture: an update on recent findings regarding responses in salmonids. Aquacult Res 41:333-344.

Krogdahl A, Bakke-McKellep AM, Baeverfjord G (2003). Effects of graded levels of standard soybean meal on intestinal structure, mucosal enzyme activities, and pancreatic response in Atlantic salmon (Salmo salar L.). Aquacult Nutr 9:361-371.

Krogdahl A, Lea TB, Olli JJ (1994). Soybean proteinase inhibitors affect intestinal trypsin activities and amino acid digestibilities in rainbow trout (Oncorhynchus mykiss). Comp Biochem Physiol A 107:215-219.

Lee CM, Sieo CC, Cheah YK, Abdullah N, Ho YW (2011). Discrimination of probiotic Lactobacillus strains for poultry by repetitive sequenced-based PCR fingerprinting. J Sci Food Agric .

Ley RE, Backhed F, Turnbaugh P, Lozupone CA, Knight RD, Gordon JI (2005). Obesity alters gut microbial ecology. Proc Natl Acad Sci U S A 102:1107011075. 
Liesack W, Weyland H, Stackerbrandt E (1991). Potential risks of gene amplification by PCR as determined by $16 \mathrm{~S}$ rDNA analys is of a mixed-culture of strict barophilic bacteria. Microb Ecol 21:191-198.

Lukwinski AT, Hill JE, Khachatourians GG, Hemmingsen SM, Hegedus DD (2006). Biochemical and taxonomic characterization of bacteria associated with the crucifer root maggot (Delia radicum). Can J Microbiol 52:197-208.

MacFarlane RD, McLaughlin JJ, Bullock GL (1986). Quantitative and qualitative studies of gut flora in striped bass from estuarine and coastal marine environments. J Wildl Dis 22:344-348.

Malathi V, Devegowda G (2001). In vitro evaluation of nonstarch polysaccharide digestibility of feed ingredients by enzymes. Poult Sci 80:302305.

Mambrini M, Roem AJ, Carvedi JP, Lalles JP, Kaushik SJ (1999). Effects of replacing fish meal with soy protein concentrate and of DL-methionine supplementation in high-energy, extruded diets on the growth and nutrient utilization of rainbow trout, Oncorhynchus mykiss. J Anim Sci 77:2990-2999.

Mansfield GS, Desai AR, Nilson SA, Van Kessel AG, Drew MD, Hill JE (2010). Characterization of rainbow trout (Oncorhynchus mykiss) intestinal microbiota and inflammatory marker gene expression in a recirculating aquaculture system. Aquacul 307:95-104. 
Margulies M, Egholm M, Altman WE, Attiya S, Bader JS, Bemben LA, et al. (2005). Genome sequencing in microfabricated high-density picolitre reactors. Nature 437:376-380.

Mark IA, Leddy MB, Bold RM, Graves AK (2011). Bacterial community composition in low-flowing river water with different sources of pollutants. FEMS Microbiol Ecol Epub:155-156.

Maron PA, Mougel C, Ranjard L (2011). Soil microbial diversity: Methodological strategy, spatial overview and functional interest. $C R$ Biol 334:403-411.

Martin-Platero AM, Valdivia E, Maqueda M, Martinez-Bueno M (2007). Fast, convenient, and economical method for isolating genomic DNA from lactic acid bacteria using a modification of the protein "salting-out" procedure. Anal Biochem 366:102-104.

McGoogan BB, Reigh RC (1996). Apparent digestibility of selected ingredients in red drum (Sciaenops ocellatus) diets. Aquacul 141:233-244.

Merrifield DL, Dimitroglou A, Foey A, Davies SJ, Baker RTM, Bogwald J, et al. (2010). The current status and future focus of probiotic and prebiotic applications for salmonids. Aquacul 302:1-18.

Millward DJ (1989). The nutritional regulation of muscle growth and protein turnover. Aquacul 79:1-28. 
Mohapatra BR, Broersma K, Mazumder A (2008). Differentiation of fecal Escherichia coli from poultry and free-living birds by (GTG)5-PCR genomic fingerprinting. Int J Med Microbiol 298:245-252.

Morris PC, Gallimore P, Handley J, Hide G, Haughton P, Black A (2005). Fullfat soya for rainbow trout (Oncorhynchus mykiss) in freshwater: Effects on performance, composition and flesh fatty acid profile in absence of hind-gut enteritis. Aquaculture 248:147-161.

Mundheim H, Aksnes A, Hope B (2004). Growth, feed efficiency and digestibility in salmon (Salmo salar L.) fed different dietary proportions of vegetable protein sources in combination with two fish meal qualities. Aquacul 237:315-331.

Namba A, Mano N, Hirose H (2007). Phylogenetic analys is of intestinal bacteria and their adhesive capability in relation to the intestinal mucus of carp. J Appl Microbiol 102:1307-1317.

Nath S, Kales S, Fujiki K, Dixon B (2006). Major histocompatibility class II genes in rainbow trout (Oncorhynchus mykiss) exhibit temperature dependent downregulation. Immunogenetics 58:443-453.

Navarrete P, Magne F, Mardones P, Riveros M, Opazo R, Suau A, et al. (2010). Molecular analysis of intestinal microbiota of rainbow trout (Oncorhynchus mykiss). FEMS Microbiol Ecol 71:148-156. 
Navarrete P, Espejo RT, Romero J (2009). Microb Ecol 57:550-561.

Naylor RL, Hardy RW, Bureau DP, Chiu A, Elliott M, Farrell AP, et al. (2009). Feeding aquaculture in an era of finite resources. Proc Natl Acad Sci U SA 106:15103-15110.

Naylor RL, Goldburg RJ, Primavera JH, Kautsky N, Beveridge MC, Clay J, et al. (2000). Effect of aquaculture on world fish supplies. Nature 405:1017-1024.

Neish AS (2009). Microbes in gastrointestinal health and disease. Gastroenterology 136:65-80.

Nordrum S, Bakke-McKellep AM, Krogdahl Å, Buddington RK (2000). Effects of soybean meal and salinity on intestinal transport of nutrients in Atlantic salmon (Salmo salar L.) and rainbow trout (Oncorhynchus mykiss). Comparative Biochemistry and Physiology Part B: Biochemistry and Molecular Biology 125:317-335.

NRC (National Research Council) (1993). Nutrient Requirements of Fish. National Academy Press: Washington, D.C.

Nubel U, Engelen B, Felske A, Snaidr J, Wieshuber A, Amann RI, et al. (1996). Sequence heterogeneities of genes encoding 16S rRNAs in Paenibacillus polymyxa detected by temperature gradient gel electrophoresis. J Bacteriol 178:5636-5643. 
Olsen RE, Hansen A, Rosenlund G, Hemre G, Mayhew TM, Knudsen DL, et al. (2007). Total replacement of fish meal with plant proteins in diets for Atlantic cod (Gadus morhua L.) II - Health aspects. Aquacul 272:612-624.

Olsen RE, Suontama J, Langmyhr E, Mundheim H, Ring Ø E, Melle W, et al. (2006). The replacement of fish meal with Antarctic krill, Euphausia superba in diets for Atlantic salmon, Salmo salar. Aquacult Nut 12:280-290.

Olsen RE, Myklebust R, Kryvi H, Mayhew TM, Ringø E (2001). Damaging effect of dietary inulin on intestinal enterocytes in Arctic charr (Salvelinus alpinus L.). Aquacult Res 32:931-934.

Ostaszewska T, Dabrowski K, Palacios ME, Olejniczak M, Wieczorek M (2005). Growth and morphological changes in the digestive tract of rainbow trout (Oncorhynchus mykiss) and pacu (Piaractus mesopotamicus) due to casein replacement with soybean proteins. Aquacul 245:273-286.

Page RD (1996). TreeView: an application to display phylogenetic trees on personal computers. Comput Appl Biosci 12:357-358.

Pearson WR, Lipman DJ (1988). Improved tools for biological sequence comparison. Proc Natl Acad Sci U S A 85:2444-2448.

Perez T, Balcazar JL, Ruiz-Zarzuela I, Halaihel N, Vendrell D, de Blas I, et al. (2010). Host-microbiota interactions within the fish intestinal ecosystem. Mucosal Immunol 3:355-360. 
Perna NT, Plunkett G,3rd, Burland V, Mau B, Glasner JD, Rose DJ, et al. (2001). Genome sequence of enterohaemorrhagic Escherichia coli O157:H7. Nature 409:529-533.

Pond MJ, Stone DM, Alderman DJ (2006). Comparison of conventional and molecular techniques to investigate the intestinal microflora of rainbow trout (Oncorhynchus mykiss). Aquacul 261:194-203.

Powell K (2003). Fish farming: eat your veg. Nature 426:378-379.

Quince C, Lanzen A, Curtis TP, Davenport RJ, Hall N, Head IM, et al. (2009). Accurate determination of microbial diversity from 454 pyrosequencing data. Nat Methods 6:639-641.

Ramirez RF, Dixon BA (2003). Enzyme production by obligate intestinal anaerobic bacteria isolated from oscars (Astronotus ocellatus), angelfish (Pterophyllum scalare) and southern flounder (Paralichthys lethostigma). Aquacul 227:417-426.

Rawls JF, Samuel BS, Gordon JI (2004). Gnotobiotic zebrafish reveal evolutionarily conserved responses to the gut microbiota. Proc Natl Acad Sci U $S$ A 101:4596-4601.

Refstie S, Storebakken T, Baeverfjord G, Roem AJ (2001). Long-term protein and lipid growth of Atlantic salmon (Salmo salar) fed diets with partial 
replacement of fish meal by soy protein products at medium or high lipid level. Aquacul 193:91-106.

Refstie S, Storebakken T, Roem AJ (1998). Feed consumption and conversion in Atlantic salmon (Salmo salar) fed diets with fish meal, extracted soybean meal or soybean meal with reduced content of oligosaccharides, trypsin inhibitors, lectins and soya antigens. Aquacul 162:301-312.

Regost C, Arzel J, Robin J, Rosenlund G, Kaushik SJ (2003). Total replacement of fish oil by soybean or linseed oil with a return to fish oil in turbot (P setta maxima): 1. Growth performance, flesh fatty acid profile, and lipid metabolism. Aquacul 217:465-482.

Ringo E (1994). The effect of chromic oxide (Cr-20-3) on faecal lipid and intestinal microflora of sea water-reared Arctic charr, Salvelinus alpinus (L.). Aquacult Fish Manage 25:341-344.

Ringo E, Sperstad S, Myklebust R., Mayhew MT, Olsen ER (2006a). The effect of dietary inulin on aerobic bacteria associated with hindgut of Arctic charr (Salvelinus alpinus L.). Aquacult Res 37:891-897.

Ringo E, Sperstad S, Myklebust R, Mayhew T, Mjelde A, Melle W, et al. (2006b). The effect of dietary krill supplementation on epithelium-associated bacteria in the hindgut of Atlantic salmon (Salmo salar L.): a microbial and electron microscopical study. Aquacult Res 37:1644-1653. 
Ringo E, Sperstad S, Myklebust R, Refstie S, Krogdahl A (2006c). Characterisation of the microbiota associated with intestine of Atlantic cod (Gadus morhua L.): The effect of fish meal, standard soybean meal and a bioprocessed soybean meal. Aquacul 261:829-841.

Ringo E, Holzapfel W (2001). Identification and characterization of Carnobacteria associated with the gills of Atlantic salmon (Salmo salar L.). Syst Appl Microbiol 23:523-527.

Ringo E, Olsen RE (1999). The effect of diet on aerobic bacterial flora associated with intestine of Arctic charr (Salvelinus alpinus L.). J Appl Microbiol 86:22-28.

Ringo E, Gatesoupe F (1998). Lactic acid bacteria in fish: a review. Aquacul 160:177-203.

Ringo E, Strom E, Tabachek J (1995). Intestinal microflora of salmonids: a review. Aquacult Res 26:773-789.

Ringo E, Strom E (1994). Microflora of Arctic charr Salvelinus alpinus: gastrointestinal microflora of free-living fish and effect of diet and salinity on intestinal microflora. Aquacult Fish Manage 25:623-629.

Robertson PAW, O'Dowd C, Burrells C, Williams P, Austin B (2000). Use of Carnobacterium sp. as a probiotic for Atlantic salmon (Salmo salar L.) and rainbow trout (Oncorhynchus mykiss, Walbaum). Aquacul 185:235-243.

\section{3}


Robinson EH, Wilson RP, Poe WE (1981). Arginine requirement and apparent absence of a lysine-arginine antagonist in fingerling channel catfish. $J$ Nutr 111:46-52.

Roeselers G, Mittge EK, Stephens WZ, Parichy DM, Cavanaugh CM, Guillemin $\mathrm{K}$, et al. (2011). Evidence for a core gut microbiota in the zebrafish. ISME J .

Romarheim OH, Skrede A, Gao Y, Krogdahl A, Denstadli V, Lilleeng E, et al. (2006). Comparison of white flakes and toasted soybean meal partly replacing fish meal as protein source in extruded feed for rainbow trout (Oncorhynchus mykiss). Aquacul 256:354-364.

Ronaghi M, Uhlen M, Nyren P (1998). A sequencing method based on real-time pyrophosphate. Science 281:363, 365.

Saha S, Roy RN, Sen KS, Ray AK (2006). Characterization of celluloseproducing bacteria from the digestive tract of tilapia, Oreochromis mossambica (Peters) and grass carp, Ctenopharyngodon idella (Valenciennes). Aquacult Res 37:380-388.

Sakata T, Sugita H, Mitsuoka T, Kakimoto D, Kadota H (1980). Isolation and distribution of obligate anaerobic bacteria from the intestines of freshwater fish. Bull Jpn Soc Sci Fish 46:1249-1255. 
Salze G, McLean E, Schwarz MH, Craig SR (2008). Dietary mannan oligosaccharide enhances salinity tolerance and gut development of larval cobia. Aquacul 274:148-152.

Sanden M, Berntssen MHG, Krogdahl A, Hemre G, Bakke-McKellep A (2005). An examination of the intestinal tract of Atlantic salmon, Salmo salar L., parr fed different varieties of soy and maize. J Fish Dis 28:317-330.

Schellenberg JJ, Links MG, Hill JE, Dumonceaux TJ, Kimani J, Jaoko W, et al. (2011a). Molecular definition of vaginal microbiota in East African commercial sex workers. Appl Environ Microbiol 77:4066-4074.

Schellenberg JJ, Links MG, Hill JE, Hemmingsen SM, Peters GA, Dumonceaux TJ (2011b). Pyrosequencing of chaperonin-60 (cpn60) amplicons as a means of determining microbial community composition. Methods Mol Biol 733:143-158.

Schellenberg JJ, Links MG, Hill JE, Dumonceaux TJ, Peters GA, Tyler S, et al. (2009). Pyrosequencing of the chaperonin-60 universal target as a tool for determining microbial community composition. Appl Environ Microbiol 75:2889-2898 .

Schloss PD, Westcott SL, Ryabin T, Hall JR, Hartmann M, Hollister EB, et al. (2009). Introducing mothur: open-source, platform-independent, communitysupported software for describing and comparing microbial communities. Appl Environ Microbiol 75:7537-7541. 
Sechi LA, Leori G, Lollai SA, Dupre I, Molicotti P, Fadda G, et al. (1999). Different strategies for molecular differentiation of Mycobacterium bovis strains isolated in Sardinia, Italy. Appl Environ Microbiol 65:1781-1785.

Seki H (1969). Marine microorganisms associated with the food of young salmon. Appl Microbiol 17:252-255.

Sheffield VC, Cox DR, Lerman LS, Myers RM (1989). Attachment of a 40-basepair G + C-rich sequence (GC-clamp) to genomic DNA fragments by the polymerase chain reaction results in improved detection of single-base changes. Proc Natl Acad Sci U S A 86:232-236.

Silva FC, Nicoli JR, Zambonino-Infante JL, Kaushik S, Gatesoupe FJ (2011). Influence of the diet on the microbial diversity of faecal and gastrointestinal contents in gilthead sea bream (Sparus aurata) and intestinal contents in goldfish (Carassius auratus). FEMS Microbiol Ecol 78:285-296.

Skrondenyte AV, Sruoga A, Butkauskas D (2006). Assessment of microbial diversity in the river trout Salmo trutta fario L. intestinal tract identified by partial 16S rRNA gene sequence analysis. Fisheries Sci 72:597-602.

Sogin ML, Morrison HG, Huber JA, Mark Welch D, Huse SM, Neal PR, et al. (2006). Microbial diversity in the deep sea and the underexplored "rare biosphere". Proc Natl Acad Sci U S A 103:12115-12120. 
Spanggaard B, Huber I, Nielsen J, Sick EB, Pipper CB, Martinussen T, et al. (2001). The probiotic potential against vibriosis of the indigenous microflora of rainbow trout. Environ Microbiol 3:755-765.

Spanggaard B, Huber I, Nielsen J, Nielsen T, Appel KF, Gram L (2000). The microflora of rainbow trout intestine: a comparison of traditional and molecular identification. Aquacul 182:1-15.

Spor A, Koren O, Ley R (2011). Unravelling the effects of the environment and host genotype on the gut microbiome. Nat Rev Microbiol 9:279-290.

Subcommittee on Fish Nutrition, National Research Council (1993). Nutrient Requirements of Fish.

Sugita H, Ito Y (2006). Identification of intestinal bacteria from Japanese flounder (Paralichthys olivaceus) and their ability to digest chitin. Lett Appl Microbiol 43:336-342.

Sugita H, Tsunohara M, Ohkoshi T, Deguchi Y (1988). The establishment of an intestinal microflora in developing goldfish (Carassius auratus) of culture ponds. Microb Ecol :333-344.

Sugita H, Tokuyama K, Deguchi Y (1985). The intestinal microflora of carp Cyprinus carpio, grass carp Ctenopharyngodon idella and tilapia Sarotherodon niloticus. Bull Jpn Soc Sci Fish 51:1325-1329. 
Suzuki MT, Taylor LT, DeLong EF (2000). Quantitative analysis of smallsubunit rRNA genes in mixed microbial populations via 5'-nuclease assays. Appl Environ Microbiol 66:4605-4614.

Svec P, Sedlacek I, Chrapava M, Vandamme P (2011). (GTG)(5)-PCR fingerprinting of lactobacilli isolated from cervix of healthy women. Folia Microbiol (Praha) 56:80-83.

Svec P, Vancanneyt M, Seman M, Snauwaert C, Lefebvre K, Sedlacek I, et al. (2005). Evaluation of (GTG)5-PCR for identification of Enterococcus spp. FEMS Microbiol Lett 247:59-63.

Tacon AGJ, Metian M (2008). Global overview on the use of fish meal and fish oil in industrially compounded aquafeeds: Trends and future prospects. Aquacul 285:146-158.

Thiessen DL, Maenz DD, Newkirk RW, Classen HL, Drew MD (2004). Replacement of fishmeal by canola protein concentrate in diets fed to rainbow trout (Oncorhynchus mykiss). Aquacult Nut 10:379-388.

Thiessen DL, Campbell GL, Adelizi PD (2003). Digestibility and growth performance of juvenile rainbow trout (Oncorhynchus mykiss) fed with pea and canola products. Aqucult Nut 9:65-75. 
Tinh NT, Dierckens K, Sorgeloos P, Bossier P (2008). A review of the functionality of probiotics in the larviculture food chain. Mar Biotechnol (NY) 10:1-12.

Trust TJ, Bull LM, Currie BR, Buckley JT (1979). Obligate anaerobic bacteria in the gastrointestinal microflora of the grass carp (Ctenopharyngodon idella), goldfish (Carassius auratus), and rainbow trout (Salmo gairdneri). J Fish Res Board Can 36:1174-1179.

Trust TJ, Sparrow RA (1974). The bacterial flora in the alimentary tract of freshwater salmonid fishes. Can J Microbiol 20:1219-1228.

Trust $\mathrm{T}$ (1974). The bacterial flora in the alimentary tract of freshwater salmonid fishes. Canadian journal of microbiology 20:1219.

Tuominen, T. R. \& Esmark, M. (2003). Food for Thought: The use of Marine Resources in Fish Feed. Report Number 02/03. WWF, Norway.

Untergasser A, Nijveen H, Rao X, Bisseling T, Geurts R, Leunissen JA (2007). Primer3Plus, an enhanced web interface to Primer3. Nucleic Acids Res 35:W714.

Vasilopoulos C, De Mey E, Dewulf L, Paelinck H, De Smedt A, Vandendriessche F, et al. (2010). Interactions between bacterial isolates from modified-atmosphere-packaged artisan-type cooked ham in view of the development of a bioprotective culture. Food Microbiol 27:1086-1094. 
Vermette CJ, Russell AH, Desai AR, Hill JE (2010). Resolution of phenotypically distinct strains of Enterococcus spp. in a complex microbial community using cpn60 universal target sequencing. Microb Ecol 59:14-24.

Verreth JAJ, Torreele E, Spazier E, Van der Sluiszen A, Rombout JHWM, Booms R, et al. (1992). The Development of a Functional Digestive System in the African Catfish Clarias gariepinus (Burchell). J World Aquacult Soc 23:286296.

Versalovic J, Schneider M, De Bruijn FJ, Lupski JR (1994). Genomic fingerprinting of bacteria using repetitive sequence-based polymerase chain reaction. Methods Mol Cell Biol 5:25-40.

Vielma J, Makinen T, Ekholm P, Koskela J (2000). Influence of dietary soy and phytase levels on performance and body composition of large rainbow trout (Oncorhynchus mykiss) and algal availability of phosphorus load. Aquacul 183:349-362.

Vogtmann H, Pfirter HP, Prabucki AL (1975). A new method of determining metabolisability of energy and digestibility of fatty acids in broiler diets. $\mathrm{Br}$ Poult Sci 16:531-534.

Walker AW, Ince J, Duncan SH, Webster LM, Holtrop G, Ze X, et al. (2011). Dominant and diet-responsive groups of bacteria within the human colonic microbiota. ISME J 5:220-230. 
Wang Q, Garrity GM, Tiedje JM, Cole JR (2007). Naive Bayesian classifier for rapid assignment of rRNA sequences into the new bacterial taxonomy. Appl Environ Microbiol 73:5261-5267.

Wasney MA, Holley RA, Jayas DS (2001). Cresol red thallium acetate sucrose inulin (CTSI) agar for the selective recovery of Carnobacterium spp. Int J Food Microbiol 64:167-174.

Watanabe T (2002). Strategies for further development of aquatic feeds. Fisheries Science 68:242-252.

Wen L, Ley RE, Volchkov PY, Stranges PB, Avanesyan L, Stonebraker AC, et al. (2008). Innate immunity and intestinal microbiota in the development of Type 1 diabetes. Nature 455:1109-1113.

Yoshimizu M, Kimura T, Sakai M (1976a). Studies on the intestinal microflora of salmonids-II. Effects of artificial transplanting from freshwater into sea water on the intestinal microilora of feeding and non-feeding fish. Bull Jpn Soc Sci Fish 42:863-873.

Yoshimizu M, Kimura T, Sakai M (1976b). Studies on the intestinal microdora of satmonids-I. The intestinal micro-flora of fish reared in freshwater and seawater. Bull Jpn Soc Sci Fish 42:91-96.

Yoshimizu M, Kimura T, Sakai M (1980). Microflora of the embryo and the fry of Salmonids. Bull Jpn Soc Sci Fish 46:967-975. 
Yu Y, Lee C, Kim J, Hwang S (2005). Group-specific primer and probe sets to detect methanogenic communities using quantitative real-time polymerase chain reaction. Biotechnol Bioeng 89:670-679.

Zhou Z, Liu Y, Shi P, He S, Yao B, Ringo E (2009). Molecular characterization of the autochthonous microbiota in the gastrointestinal tract of adult yellow grouper (Epinephelus awoara) cultured in cages. Aquacul 286:184-189. 